\title{
Dynamics of nearly inviscid Faraday waves in almost circular containers
}

\author{
M. Higuera , E. Knobloch , J.M. Vega \\ E.T.S.I Aeronáticos, Iniversidad Politécnica de Madrid, Plaza Cardenal Cisneros 3, 28040 Matrid. Spain \\ Deparment of Applied Mahemalics. Liniversily of Leeds, Leeds LS2 9JT, UK \\ Department of Physics, Liniversily of California, Berkeley, CA 94720. USA
}

\begin{abstract}
Parametrically driven surface gravity-capillary waves in an elliptically distorted circular cylinder are studicd. In the nearly inviscid regime, the waves couple to a streaming flow driven in oscillatory viscous boundary layers. In a cylindrical container, the streaming flow couples to the spatial phase of the waves, but in a distorted cylinder, it couples to their amplitudes as well. This coupling may destabilize pure standing oscillations, and lead to complex time-dependent dynamics at onset. Among the new dynamical behavior that results are relaxation oscillations involving abrupt transitions between standing and cuasiperiodic oscillations, and cxhibiting 'canards'.
\end{abstract}

Keywords: Faraday oscillations; Forced symmetry breaking; Global bifurcations; Relaxation oscillations; Canards

\section{Introduction}

Surface gravity-capillary waves exhibit a wide variety of pattern-forming phenomena, depending both on the mode of excitation and on the size of the domain relative to the wavelength selected by the driving frequency. In large domains recent work has focused on two-frequency forcing [1], in which the two frequency components select distinct wavenumbers. These cxperiments, generally carricd out in quite viscous liquids, have revealed various superlattice patterns, as well as nonperiodic states resembling quasipatterns $[12,27,28,1]$. In these experiments, the frequencies used are in (low order) resonance, so that the forcing accelcration remains periodic in time, with the 
frequency ratio, their relative phase, and their relative contribution to the forcing providing parameters to be tuned in the experiment. Existing theory has been successful in explaining a number of the experimental observations $[42,37,39]$, but for reasons explained below such theory is generally restricted to highly viscous liquids. This is because in nearly inviscid liquids the behavior is complicated by the presence of a mean flow, also known as a streaming flow, driven by time-averaged Reynolds stresses in oscillatory viscous boundary layers near rigid walls and the free surface $[40,31,43]$. These mean flows in turn interact with the waves that produce them, leading to a description of the Faraday system in terms of amplitude equations coupled to a Navier-Stokes-like equation for the mean flow with boundary conditions obtained by matching to the oscillatory boundary layers [43]. These equations represent a new class of pattem-forming dynamical systems.

The new terms in the amplitude equations are formally of cubic order, indicating that the effects of the mean flow cannot be neglected, even in the limit of vanishing viscosity. The resulting equations have thus far been studied only in the simplest cases: with single frequency forcing, periodic boundary conditions and one horizontal dimension. When the spatial period is comparable to the wavelength selected by the parametric forcing the coupled amplitudemean flow cquations simplify: all solutions are attracted to standing waves of constant amplitude, and the streaming flow only advects the spatial phase of these waves; this phase in turn appears in the boundary conditions for the streaming flow, giving rise to a pair of simpler coupled phase-mean flow equations. Analysis of these equations reveals that the coupling to the streaming flow is significant, and can lead to a number of new instabilities, which are absent if the streaming flow is neglected [33]. These include parity-breaking instabilities that lead to pattern drift, and oscillatory instabilitics that produce oscillations in the spatial phase of the pattern. In larger domains amplitude inhomogeneities can develop and these also couple to the mean flow [30].

In the present paper we examine this type of dynamical system in three dimensions. The structure of the resulting equations in various small aspect ratio domains has recently been determined [22]. Of the domains considered a circular domain is of particular interest. If the domain is precisely circular the amplitude of the standing waves produced by the parametric driving decouples from the mean flow, and the streaming flow couples to the spatial phase of the pattern only, as in ref. [33]. However, as pointed out in [22], the situation changes dramatically when the shape of the container is perturbed. In the case of a slightly elliptical container two types of standing waves, oriented along the major and minor axes of the ellipse, come in in close succession as the amplitude of the parametric excitation increases, and these may interact already at small amplitude, producing mixed modes which are much more efficient at driving a streaming flow. In this case, the strcaming flow couples to the two amplitudes, as well as to the spatial phase of the resulting pattern. This interaction with the streaming flow is responsible for a new class of dynamical behavior that now involves the amplitudes as well. In particular, we demonstrate in this paper that it can lead to relaxation oscillations of a particularly interesting type: oscillations that switch from single frequency standing waves to two-frequency waves and back. Under appropriate conditions these relaxation oscillations can cxhibit the so-called "canard' phenomenon in which the system follows nominally unstable solutions in the slow phase. Various global bifurcations are located as well. of which perhaps the most interesting is responsible for the appearance of chaotic dynamics right at threshold of the primary instability.

Our calculations are based on the assumption that the effective Rcynolds number of the streaming llow, suitably defined, remains small. This assumption permits us to project the Navier-Stokes-like equation for the streaming flow onto the dominant spatial eigenfunction, replacing it by a single ordinary differential cquation for the evolution of the amplitude of this eigenfunction. The coupling of this viscous mode to the amplitude of the competing standing waves is relained as a free parameter; the remaining nonlinear cocfficient values are taken (mostly) from a paper by Miles [34] for an inviscid fluid in a circular cylinder of aspect ratio $R \approx 0.66$ with a free contact line.

The paper is organized as follows. In Section 2, we summarize the derivation of the coupled amplitude-streaming flow equations for this system, and the conditions for their validity. In Section 3, we discuss the basic properties of these cquations, and identify four regions in parameter space where these cquations are valid but new types of dynamical behavior may be expected. In Section 4 , we explore numerically the behavior of the coupled amplitudestreaming flow equations in these regions and offer, in each case, a dynamical systems explanation of the observed 
dynamics. The paper concludes with a discussion of the implication of our results, both for dynamical systems theory and for future experiments on the Faraday system.

\section{Coupled amplitude-streaming flow equations}

We consider nonaxisymmetric Faraday waves in a slightly elliptical container. The ellipticity breaks the $O(2)$ symmetry of the container and picks out standing oscillations with nodes along either the major or the minor axis of the cllipsc. A convenient way of understanding this selection is to formulate the problem first in a circular container, treating the ellipticity as a small perturbation, comparable to the small damping from the viscous boundary layers (and the llow in the bulk) and the small forcing amplitude required to overcome it. In the unforced case, the free oscillations of the fluid in such a container are most easily described in terms of clockwise and counterclockwise rotating waves, and we therefore use the amplitudes $A_{ \pm}$of these waves to describe the dy namics of the system. In a circular container, the parametric forcing couples these waves, resulting in a reflection-symmetric standing oscillation with arbitrary orientation. Because of this symmetry only the phase of the standing wave couples to the streaming flow. However, as soon as the shape of the container is perturbed from circular both the phase and the amplitudes couple to the streaming flow. This coupling takes place via the difference in frequency between the oscillations along the two principal axes $[22\rceil$ and has a dramatic impact on the dynamics.

Since we are treating departures from circular symmetry as a perturbation we can use cylindrical coordinates $(r, O, z)$, and consider a circular cyclinder of height $h$ and radius $R h$. In these coordinates, the coupled amplitudestrcaming llow equations derived by Higuera el al. [22], suitably scalcd, take the form

$$
\begin{aligned}
& A_{ \pm}^{\prime}(\tau)=-(1+\mathrm{i} \Gamma) A_{ \pm}+\mathrm{i} \Lambda A_{\mp}+\mathrm{i}\left(\alpha_{1}\left|A_{ \pm}\right|^{2}+\alpha_{2}\left|A_{\mp}\right|^{2}\right) A_{ \pm} \\
&+\mathrm{i} \mu \bar{A}_{\mp} \mp \mathrm{i} \Omega \int_{-1}^{0} \int_{0}^{2 \pi} \int_{0}^{R} g(r, z) \boldsymbol{u} \cdot \boldsymbol{e}_{\theta} r \mathrm{~d} r \mathrm{~d} \theta \mathrm{d} z A_{ \pm}, \\
& \frac{\partial \boldsymbol{u}}{\partial \tau}-\left[\boldsymbol{u}+\boldsymbol{G}\left(A_{+}, A_{-}\right)\right] \times(\nabla \times \boldsymbol{u})=-\nabla p+R e^{-1} \Delta \boldsymbol{u},
\end{aligned}
$$

with $\nabla \cdot u=0$. In these equations, $u$ denotes the streaming flow, $\Omega$ is a known constant, and the quantities $\Gamma, \mu$ and $\Lambda$ are proportional to the deluning, forcing amplitude and ellipticity of the container, respectively. The detuning takes into account the mismatch between half the forcing frequency and the natural frequency of inviscid oscillations, and includes the frequency shift due to viscosity, while $A$ is proportional to the frequency difference $\Omega_{1}-\Omega_{2}$ between inviscid oscillations along the two principal axes of the container. The time has been scaled by the viscous damping time $\delta \equiv \gamma_{1} C_{\mathrm{g}}^{1 / 2}+\gamma_{2} C_{\mathrm{g}}$, where $C_{\mathrm{g}} \equiv \nu\left(g h^{3}+T h / \rho\right)^{-1 / 2} \ll 1$, and $\gamma_{1}$ and $\gamma_{2}$ are known constants that depend on the excited mode. This scaling is responsible for the appearance of the Reynolds number

$$
R e=\frac{\gamma_{1} C_{\mathrm{g}}^{\mathrm{l} / 2}+\gamma_{2} C_{\mathrm{g}}}{C_{\mathrm{g}}}
$$

in the Navier-Stokes-like Eq. (2) for the streaming flow. This Reynolds number is formally of order one [32,43], but can in fact be both large and small. For cxample, if the container is not decp the damping of the waves is dominated by the Stokes boundary layers and $R e \sim \gamma_{1} / \sqrt{C_{\mathrm{g}}}$. while in a deep container both terms (i.e., $\gamma_{1} C_{\mathrm{g}}^{1 / 2}+\gamma_{2} C_{\mathrm{g}}$ ) contribute to the damping. Calculations show that for the first few modes $\gamma_{2} / \gamma_{1} \sim 10^{2}$ [32] so that systems with $C_{\mathrm{g}} \lesssim 10^{-4}$, such as watcr or silicon oils in centimeter-decp containers, have Rcynolds numbers $\operatorname{Re}=O\left(1 / \sqrt{C_{\mathrm{g}}}\right)$. In these systems, therefore, the streaming is only weakly damped and hence is easily driven by time-averaged Reynolds stresses. 
Eq. (2) also contains a Stokes drift term $G$ given by

$$
\boldsymbol{G}=\left(\left|\Lambda_{-}\right|^{2}-\left|\Lambda_{+}\right|^{2}\right) g(r, 0) \boldsymbol{e}_{i},
$$

and is to be solved subject to the boundary conditions

$$
\begin{aligned}
\boldsymbol{u}= & {\left[\varphi_{1} A_{+} \bar{A}_{-} \mathrm{e}^{2 \mathrm{i} m \theta}+\text { c.c. }+\varphi_{2}\left(\left|A_{+}\right|^{2}+\left|A_{-}\right|^{2}\right)\right] \boldsymbol{n}_{0} \times \boldsymbol{e}_{\theta}+\left[i \varphi_{3} A_{+} \bar{A}_{-} \mathrm{e}^{2 \mathrm{i} m \theta}\right.} \\
& \left.+ \text { c.c. }+\varphi_{4}\left(\left|A_{-}\right|^{2}-\left|A_{+}\right|^{2}\right)\right] \boldsymbol{e}_{\theta}
\end{aligned}
$$

on either $r=R$ or $z=-1$, and

$$
\boldsymbol{u} \cdot \boldsymbol{e}_{z}=\frac{\partial \boldsymbol{u}}{\partial z} \cdot \boldsymbol{e}_{r}=0, \quad \frac{\partial \boldsymbol{u}}{\partial z} \cdot \boldsymbol{e}_{\theta}=\varphi_{5}\left(\left|A_{-}\right|^{2}-\left|A_{+}\right|^{2}\right)
$$

on $z=0$. Here $n_{0}$ denotes the outward unit normal, and the scalar functions $\varphi_{1}, \ldots, \varphi_{5}$ and $g$ are all real, independent of $\theta$ and computable in terms of the components of the cxciled inviscid linear mode of the system [22]. For a pinned contact line the coefficients $\gamma_{1}$ and $\gamma_{2}$ in (3) and the corresponding inviscid eigenfunctions have been calculated in [32]. while Miles [34] has calculated the coefficients $\alpha_{1}$ and $\alpha_{2}$ of the nonlinear terms for a particular case with a free contact line (sce Fig. 1). In the following we generally use Miles' results for $h=14.1 \mathrm{~cm}$ and $R h=9.24 \mathrm{~cm}$, viz. $\alpha_{1}=0.4, \alpha_{2}=-2.58$ (Cases I-III). These values correspond to an experiment of Funakoshi and Inoue [14], although in this case the waves were excited directly by lateral vibration, rather than parametrically. The results in Case IV are obtained for $\alpha_{1}=0.48, \alpha_{2}=-0.58$.

It is important to observe that in these equations all coefficients are formally of order one. In particular, the forcing of the streaming flow remains finite even in the limit of vanishing viscosity, as originally noted by Schlichting [40] and Longuet-Higgins [31]. The boundary conditions $(5,6)$ show that its magnitude is in general of order $\left(\left|A_{+}\right|^{2},\left|A_{-}\right|^{2}\right)$, and hence of order $\mu-\mu_{0}$, where $\mu_{0}$ is the threshold for the onset of the Faraday instability. Note also that a nonvanishing streaming flow is associated even with standing waves $\left(\left|A_{+}\right|=\left|A_{-}\right|\right)$, although for such waves $\boldsymbol{u} \cdot \boldsymbol{e}_{\theta}$ changes sign under reflection $\theta \rightarrow-O$ and the last term in Eq. (1) vanishes. Thus for standing waves the streaming flow decouples from the amplitudes, although it may be involved in triggering instabilitics of such waves.

\subsection{The circular container}

As already mentioned, when $A=0$ the streaming flow decouples from the equations for the amplitudes. To see this we consider waves with azimuthal wavenumber $m$, and write

$$
A_{ \pm}=B_{ \pm} \mathrm{c}^{-\mathrm{i} m 0_{0}(r)}
$$

to obtain

$$
B_{ \pm}^{\prime}(\tau)=-(1+\mathrm{i} \Gamma) B_{ \pm}+\mathrm{i}\left(\alpha_{1}\left|B_{ \pm}\right|^{2}+\alpha_{2}\left|B_{\mp}\right|^{2}\right) B_{ \pm}+\mathrm{i} \mu \bar{B}_{\mp}
$$

and

$$
O_{0}^{\prime}(\tau)=(\Omega / m) \int_{-1}^{0} \int_{0}^{2 \pi} \int_{0}^{R} g(r, z) \boldsymbol{u} \cdot \boldsymbol{e}_{\theta} r \mathrm{~d} r \mathrm{~d} \varrho \mathrm{d} z .
$$

These equations provide the simplest description of nearly inviscid Faraday waves in $O(2)$-symmetric systems and all their solutions converge to reflection-symmetric steady states of the form

$$
B_{ \pm}=R_{0} \mathrm{e}^{\mathrm{i} n \theta_{0}^{0}}
$$



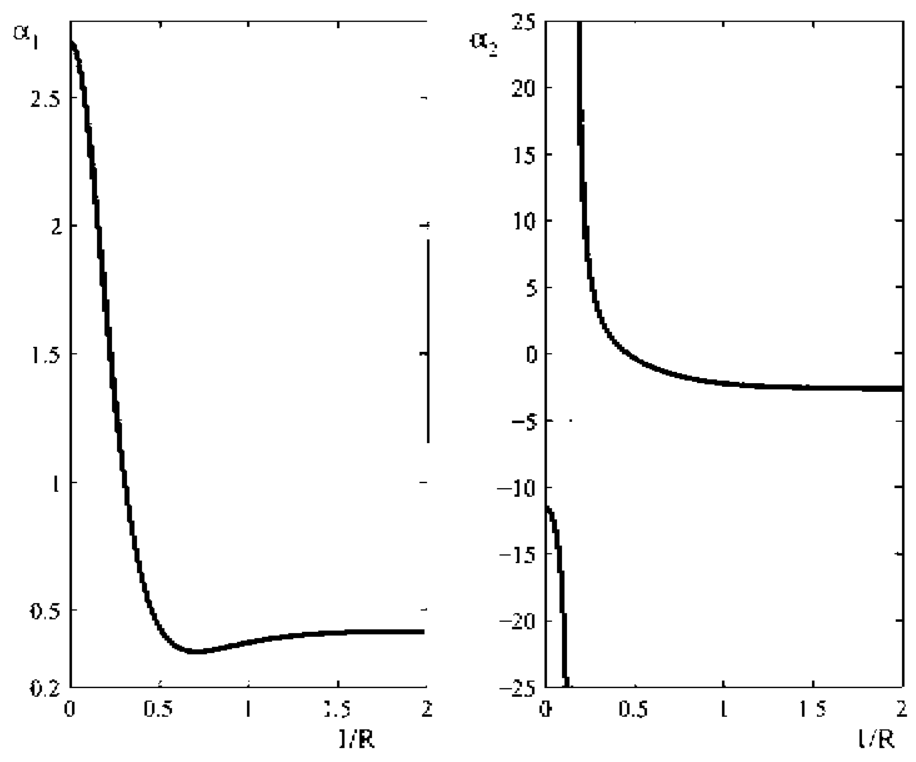

Fig. 1. The coefficients $c \chi_{1}, \chi_{2}$ for a $m=1$ inviscid mode with a free contact line as a function of the aspect ratio $R$. Here $\alpha_{1}=-A-B$, $\alpha_{2}=-A+B$, where $A$ and $B$ are computed by Miles [34].

i.c., to standing waves, and only the spatial phase $\theta_{0}$ couples to the streaming flow, as described by Eq. (9) and

$$
\frac{\partial \boldsymbol{u}}{\partial \tau}-\boldsymbol{u} \times(\nabla \times \boldsymbol{u})=-\nabla p+R e^{-1} \Delta \boldsymbol{u}, \quad \nabla \cdot u=0,
$$

subject to

$$
\boldsymbol{u}=2 R_{0}^{2}\left[\varphi_{1} \cos \left[2 m\left(\theta-\theta_{0}\right)\right]+\varphi_{2}\right] n_{0} \times \boldsymbol{e}_{\theta}-2 R_{0}^{2} \varphi_{\mathcal{3}} \sin \left[2 m\left(\theta-\theta_{0}\right)\right] \boldsymbol{e}_{\theta}
$$

on either $r=R$ or $z=-1$, and

$$
\boldsymbol{u} \cdot \boldsymbol{e}_{z}=\frac{\partial \boldsymbol{u}}{\partial z} \cdot \boldsymbol{e}_{r}=\frac{\partial u}{\partial z} \cdot e_{\theta}=0
$$

on $z=0$. The (constant) arbitrary phase $\theta_{0}^{0}$ appearing in (10) has been eliminated by an appropriate rotation. Eqs. (9), (11)-(13) possess, for all $R_{0}^{2}$, reflection-symmetric steady states of the form $\boldsymbol{u}=\boldsymbol{u}^{\mathrm{s}}\left(r, \theta-\theta_{0}, z\right), \theta_{0}=$ constant, with $\boldsymbol{u}^{\mathrm{s}}(r, \theta, z) \cdot \boldsymbol{e}_{\theta}=-\boldsymbol{u}^{\mathrm{s}}(r,-\theta, z) \cdot \boldsymbol{e}_{\theta}$; note that there is a whole family of such states, obtained by an arbitrary rotation. For small $R_{0}^{2}$ the cxistence and (orbital) asy mptotic stability of these states can be ascertained analytically. It turns out that these states can lose stability at finite $R_{0}$ either through a parity-breaking bifurcation giving rise to uniformly drifting spatially uniform standing waves (such as those observed in Faraday experiments in annular containers [10]), or via a symmetry-breaking Hopf bifurcation that produces so-called direction-reversing waves [29]. In the latter case, the standing waves drift alternately clockwise and counterclockwise but their mean location remains fixed. Solutions of this type have been found in a two-dimensional Cartesian geometry with periodic boundary conditions, and represent the instability that sets in at smallest amplitude [33]. Neither instability is present if the coupling to the streaming flow is ignored. 


\subsection{Nearly circular cylinder}

As soon as $\Lambda \neq 0$ the symmetry of the problem is reduced to the group $D_{2}$ generated by

$$
\Lambda_{ \pm} \rightarrow-\Lambda_{ \pm}, \quad 0 \rightarrow \theta+\pi, \quad \text { and } \quad \Lambda_{+} \leftrightarrow \Lambda_{-}, \quad 0 \rightarrow-0, \quad \boldsymbol{u} \cdot \boldsymbol{e}_{\theta} \rightarrow-\boldsymbol{u} \cdot \boldsymbol{e}_{\theta},
$$

and the streaming flow couples to the amplitudes as well. The description of this coupling becomes simpler when the effective Reynolds number of the streaming flow is small (i.e., $\mu-\mu_{0}$ is small) for then the non-potential term $-\boldsymbol{u} \times(\nabla \times \boldsymbol{u})$ in Eq. (2) is negligible. In fact this approximation remains qualitatively useful even for larger Reynolds numbers. The absence of nonlinear terms allows us to isolate the part of the streaming flow velocity that contributes to the nonlocal term in Eq. (1), by decomposing the streaming flow variables as

$$
(\boldsymbol{u}, p)=\left(v(r, z, \tau) e_{\theta}, 0\right)+(\hat{u}, p), \quad \text { where } \int_{0}^{2 \pi} \hat{u} \cdot e_{\theta} \mathrm{d} \theta \equiv 0 .
$$

Thus,

$$
\begin{aligned}
& \begin{array}{l}
\Lambda_{ \pm}^{\prime}(\tau)=-(1+\mathrm{i} \Gamma) \Lambda_{ \pm}+\mathrm{i} \Lambda \Lambda_{\mp}+\mathrm{i}\left(\alpha_{1}|\Lambda \pm|^{2}+\alpha_{2}\left|\Lambda_{\mp}\right|^{2}\right) \Lambda_{ \pm} \\
\quad+\mathrm{i} \mu \bar{\Lambda}_{\mp} \mp 2 \pi \mathrm{i} \Omega \int_{-1}^{0} \int_{0}^{R} g(r, z) v(r, z, \tau) r \mathrm{~d} r \mathrm{~d} z \Lambda_{ \pm},
\end{array} \\
& v_{\tau}=R^{-1}\left(v_{r}+r^{-1} v_{r}-r^{-2} v+v_{z z}\right) \quad \text { if } \quad 0<r<R, \quad-1<z<0, \\
& v=0 \quad \text { as } r \rightarrow 0, \\
& v=\varphi_{4}\left(\left|A_{-}\right|^{2}-\left|A_{+}\right|^{2}\right) \quad \text { on either } r=R \text { or } z=-1, \\
& v_{z}=\varphi_{5}\left(\left|A_{-}\right|^{2}-\left|A_{+}\right|^{2}\right) \quad \text { on } z=0,
\end{aligned}
$$

and the axisymmetric part of the streaming flow vanishes for standing waves $\left(\left|\Lambda_{+}\right|=\left|\Lambda_{-}\right|\right)$.

Although the above model can be integrated numerically by relatively inexpensive methods we simplify it further by expanding the axisymmetric component of the streaming flow velocity, $v$, in terms of purely azimuthal hydrodynamic modes. These satisfy the eigenvalue equation

$$
\begin{aligned}
& \lambda V=V_{r r}+r^{-1} V_{r}-r^{-2} V+V_{z z} \quad \text { if } \quad 0<r<R, \quad-1<z<0, \\
& V=0 \text { as } r \rightarrow 0, \quad V=\varphi_{4} \text { on either } r=R \text { or } z=-1, \quad V_{z}=\psi_{5} \text { on } z=0 .
\end{aligned}
$$

The eigenvalues $\lambda$ are all real and negative. If only the first such mode is retained, we obtain

$$
\begin{aligned}
& \Lambda_{ \pm}^{\prime}(\tau)=-(1+\mathrm{i} \Gamma) \Lambda_{ \pm}+\mathrm{i} \Lambda \Lambda_{\mp}+\mathrm{i}\left(\alpha_{1}\left|\Lambda_{ \pm}\right|^{2}+\alpha_{2}\left|\Lambda_{\mp}\right|^{2}\right) \Lambda_{ \pm}+\mathrm{i} \mu \bar{\Lambda}_{\mp} \mp \mathrm{i} \gamma v_{1} \Lambda_{ \pm}, \\
& v_{1}^{\prime}(\tau)=\varepsilon\left(-v_{1}+\left|\Lambda_{-}\right|^{2}-\left|\Lambda_{+}\right|^{2}\right),
\end{aligned}
$$

where $\varepsilon=-\lambda_{1} R e^{-1}>0$, and $\lambda_{1}<0$ is the first purely azimuthal hydrodynamic cigenvalue. In the following we define new variables

$$
X=\mathrm{i}\left(A_{+}-A_{-}\right) / 2, \quad Y=\left(A_{+}+A_{-}\right) / 2, \quad v=-v_{1} / 2,
$$

and rewrite Eqs. (23)-(24) in the more useful form

$$
\begin{aligned}
& X^{\prime}=-(1+\mathrm{i}(\Gamma+\Lambda)) X+\mathrm{i}\left(\left(\alpha_{1}+\alpha_{2}\right)|X|^{2}+2 \alpha_{1}|Y|^{2}\right) X-\mathrm{i}\left(\alpha_{1}-\alpha_{2}\right) \bar{X} Y^{2}+\mathrm{i} \mu \bar{X}-2 \gamma v Y, \\
& Y^{\prime}=-(1+\mathrm{i}(\Gamma-\Lambda)) Y+\mathrm{i}\left(\left(\alpha_{1}+\alpha_{2}\right)|Y|^{2}+2 \alpha_{1}|X|^{2}\right) Y-\mathrm{i}\left(\alpha_{1}-\alpha_{2}\right) \bar{Y} X^{2}+\mathrm{i} \mu \bar{Y}+2 \gamma v X,
\end{aligned}
$$




$$
v^{\prime}=\varepsilon(-v+\mathrm{i}(\bar{X} Y-X \bar{Y}))
$$

This form of the equations makes it clear not only that the (axisymmetric part of the) streaming flow vanishes if either $X=0$ or $Y=0$, i.e., for pure standing waves, but also that both modes must be present in order to drive such a flow, i.e., all instabilities of standing waves within Eqs. (26)-(28) will be due to mode interaction, at least when $\Lambda \neq 0$.

Eqs. (26)-(28) constitute the basic system studied in the remainder of this paper, and we investigate its properties for small values of $\varepsilon$. If $\varepsilon$ is too large the mean flow becomes slaved to the amplitudes, and Eqs. (26)-(28) become instead

$$
\begin{aligned}
& X^{\prime}=-(1+\mathrm{i}(I+\Lambda)) X+\mathrm{i}\left(\left(\alpha_{1}+\alpha_{2}\right)|X|^{2}+2\left(\alpha_{1}+\gamma\right)|Y|^{2}\right) X-\mathrm{i}\left(\alpha_{1}-\alpha_{2}+2 \gamma\right) \bar{X} Y^{2}+\mathrm{i} \mu \bar{X}, \\
& Y^{\prime}=-(1+\mathrm{i}(\Gamma-\Lambda)) Y+\mathrm{i}\left(\left(\alpha_{1}+\alpha_{2}\right)|Y|^{2}+2\left(\alpha_{1}+\gamma\right)|X|^{2}\right) Y-\mathrm{i}\left(\alpha_{1}-\alpha_{2}+2 \gamma\right) \bar{Y} X^{2}+\mathrm{i} \mu \bar{Y},
\end{aligned}
$$

i.e., amplitude equations for the Faraday system of the usual kind, but with coefficients modified by the presence of the mean flow according to $\alpha_{1} \rightarrow \alpha_{1}+\gamma, \alpha_{2} \rightarrow \alpha_{2}-\gamma$. The resulting equations form a special case of the sy stem studied by Dangelmayr and Knobloch [9]. When $\varepsilon$ is sufficiently small the streaming flow $v$ participates actively in the dy namics and permits behavior that would not otherwise occur. This occurs when the mean flow is sufficiently weakly damped, i.c., when $\left|\lambda_{1}\right|$ is sufficiently small or Re sufficiently large.

\section{Analytical results}

Eqs. (26)-(28) are equivariant with respect to the group $D_{2}$ generated by the two reflections

$$
R_{1}:(X, Y, v) \rightarrow(-X, Y,-v), \quad R_{2}:(X, Y, v) \rightarrow(X,-Y,-v) .
$$

These symmetries will prove to be of crucial importance in the analysis that follows.

We begin by cxamining the stcady states and their stability properties. The steady states are of two types, hereafter pure modes associated with vanishing streaming flow, and mixed modes which are accompanied by a nonzcro strcaming flow. These are distinguished by their symmetries.

\subsection{Pure modes $\left(P_{ \pm}\right)$}

The two possible pure modes are given by $P_{+} \equiv(0, Y, 0)=\left(0, R_{+} \mathrm{e}^{\mathrm{i} \phi_{+}}, 0\right)$ and $P_{-} \equiv(X, 0,0)=\left(R_{-} \mathrm{e}^{\mathrm{i} \phi_{-}}, 0,0\right)$, with $P_{+}$invariant under $R_{1}$ and $P_{-}$under $R_{2}$. The amplitudes of these modes satisfy

$$
P_{ \pm}: 1+\left(I^{\prime} \mp \Lambda-\left(\alpha_{1}+\alpha_{2}\right) R_{ \pm}^{2}\right)^{2}=\mu^{2}
$$

The $P_{ \pm}$are both pure parity standing waves, one with a nodal line along the major axis of the container and the other along the minor axis. When $\Lambda=0$ and the container is perfectly circular the pure modes are part of a whole circle of standing oscillations.

The $P_{+}$and $I_{-}$- solutions bifurcate from the trivial state at

$$
\mu_{0+}=\left(1+(I-\Lambda)^{2}\right)^{1 / 2} \text { and } \mu_{0-}=\left(1+(I+\Lambda)^{2}\right)^{1 / 2},
$$

respectively. The bifurcation to $P_{ \pm}$is supercritical if $(\Gamma \mp \Lambda)\left(\alpha_{1}+\alpha_{2}\right)<0$.

Linearization about the pure modes identifies the stability properties of these states. These fall into one of two classes: the eigenvector may respect the symmetry of the solution, or it may break it. A steady state bifurcation of the former type corresponds to saddle-node bifurcations ( $\mathrm{SN}$ ), while a steady state bifurcation of the latter type is a pitchfork that produces a pair of mixed modes, i.e., steady states that do not have any symmetry. In the following we 
refer to this bifurcation as a symmetry-breaking bifurcation (SB). A simple calculation shows that these bifurcations occur at

$$
\begin{aligned}
& \mathrm{SN}: R_{ \pm}^{2}=\frac{I^{\prime} \mp \Lambda}{\alpha_{1}+\alpha_{2}}, \\
& \mathrm{SB}: R_{ \pm}^{2}=\frac{\Gamma}{2\left(\alpha_{1}+\gamma\right)}, \quad \text { if } \quad \Gamma \Lambda \neq 0,
\end{aligned}
$$

respectively. We emphasize that SB produces steady solutions of the form $(X, Y, v), X Y v \neq 0$, in contrast to the parity-breaking bifurcation that occurs when $A=0$ and produces a drifing pure mode.

The pure modes $P_{ \pm}$may also experience Hopf bifurcation. Only one type, a symmetry-breaking Hopf bifurcation, is possible and takes place al

$$
R_{ \pm}^{2}=\frac{4 I \Lambda \pm 2 \varepsilon \pm \varepsilon^{2}}{4 \Lambda\left(2 \alpha_{1}-\varepsilon \gamma\right)}
$$

provided that $\gamma \Lambda \neq 0$. This requirement follows immediately from the expression for the oscillation frequency $\omega_{ \pm}$,

$$
\omega_{ \pm}^{2}=-\varepsilon^{2} \mp 4 \gamma \Lambda \varepsilon R_{ \pm}^{2}>0 .
$$

Thus, at most one pure mode can lose stability at a symmetry-breaking Hop f bifurcation, $P_{+}$if $\gamma \Lambda<0$, and $P_{-}$if $\gamma \Lambda>0$. Note that this instability involves the excitation of the streaming flow $(\gamma \neq 0)$ and requires a finite ellipticity of the container. The oscillations that result resemble trapped direction-reversing waves, oscillating about the major or minor axes, but exhibit no drift. Such states correspond to two-frequency Faraday oscillations.

\subsection{Mixed modes (M)}

The mixed modes $M \equiv(X, Y, v)=\left(R_{-} \mathrm{e}^{\mathrm{i} \phi_{-}}, R_{+} \mathrm{e}^{\mathrm{i} \phi_{+}}, v\right)$ satisfy

$$
\begin{aligned}
& E \equiv R_{+}^{2}+R_{-}^{2}=\frac{\Gamma}{2\left(\alpha_{1}+\gamma\right)}, \\
& \left(E\left(\alpha_{1}+\alpha_{2}\right)-\Gamma\right)^{2}+\left(1+\Lambda^{2}+\Lambda\left(\alpha_{2}-\alpha_{1}-2 \gamma\right) L\right)^{2}=\left(1+\Lambda^{2}\right) \mu^{2}, \\
& \tan \left(\phi_{+}-\phi_{-}\right)=\Lambda, \quad \cos ^{2}\left(\phi_{+}+\phi_{-}\right)=\frac{\left(E\left(\alpha_{1}+\alpha_{2}\right)-I\right)^{2}}{\mu^{2}\left(1+\Lambda^{2}\right),}
\end{aligned}
$$

where $L \equiv R_{+}^{2}-R_{-}^{2}$. Since these modes have no symmetry, the linearization about them does not block-diagonalize, and the stability analysis must in general be done numerically. However, it is possible to show that the mixed modes lose stability at a saddle-node bifurcation when

$$
\mu^{2}\left(1+\Lambda^{2}\right)\left(\alpha_{1}+\gamma\right)^{2}-\Gamma^{2}\left(\alpha_{1}-\alpha_{2}+2 \gamma\right)^{2}=0,
$$

a result that also follows from Eqs. $(38,39)$. These modes can also undergo a Hopf bifurcation. In contrast to the pure modes such a bifurcation is possible even when $\gamma=0$, but then occurs only if $P_{+}$and $P_{-}$bifurcate in opposite directions. However, if $\gamma \neq 0$, a Hopf bifurcation may occur along the mixed mode branch even when it connects two supercritical pure modes.

The stability results for the $P_{ \pm}$and $M$ solutions described above are illustrated in Fig. 2 for four representative sets of parameter values. In Fig. 2a, both pure mode branches bifurcate supercritically, with the $P_{-}$branch bifurcating before the $P_{+}$branch. The $P_{-}$states are therefore stable at small amplitude and remain so until a symmetry-breaking bifurcation at $\mathrm{SB}$, where stability is transferred to the mixed modes $M$. These in turn lose stability at a Hopf bifurcation prior to the end of the $M$ branch on the $P_{+}$branch. The $P_{+}$solutions above 
SB have two unstable eigenvalues and acquire stability only at larger amplitude, at another Hopf bifurcation. A stability gap, where time-dependent solutions may be expected, is therefore present between the two Hopf bifurcations $H$.

Fig. $2 \mathrm{~b}$ shows a case in which a symmetry-breaking Hopf bifurcation occurs below the symmetry-breaking steady state bifurcation on the $P_{-}$branch. Since the latter is subcritical the resulting mixed modes have initially three unstable eigenvalues but acquire stability after a saddle-node bifurcation, followed by a Hopf bifurcation. Once stable they remain so until their termination point on the $P_{+}$branch, where they transfer stability to the $P_{+}$ branch. Once again time-dependent behavior is expected in the region between the two Hopf bifurcations $H$.

In Fig. 2c, both pure mode branches bifurcate subcritically. The $P_{-}$branch bifurcates first and acquires stability at a saddle-node bifurcation before losing it again at larger amplitude at a symmetry-breaking steady state bifurcation SB. The $P_{+}$branch is never stable, and neither are the mixed modes connecting the pure mode branches. Here time-dependent states set in beyond the symmetry-breaking bifurcation on the $P_{-}$branch.

Finally, in Fig. 2d the parameters $\Gamma$ and $\Lambda$ are small, and the pure modes $P_{ \pm}$come in in close succession and bifurcate almost vertically (sec Eq. (32)). The figure shows that this time it is the $P_{+}$branch that comes in first, and that it bifurcates slightly subcritically with all three of the possible secondary instabilities present. Since the Hopf bifurcation occurs below the saddle-node bifurcation the $P_{+}$state has two unstable eigemvalues above the saddlenode, one of which passes through zero at SB. It follows that the mixed mode states created at SB have initially two unstable eigenvalues, i.e., that the bifurcation is subcritical. In contrast, the $P_{-}$state bifurcates supercritically, and has one unstable cigenvalue near onset. It acquires stability at $\mathrm{SB}$, indicating that the bifurcation from $P_{-}$to the mixed modes is supercritical, and hence that the mixed modes are stable near $P_{-}$. It follows that there must be a Hopf bifurcation along the mixed mode branch, at $\mu=\mu_{H}^{M}$ say, and indeed our calculations confirm that this is so. The presence of this bifurcation is important in what follows since it implies that Eqs. (26)-(28) have no stable nontrivial fixed points for $\mu<\mu_{H}^{M}$. Consequently, the first state observed once $\mu$ exceeds the threshold value $\mu_{0}$ must be a time-dependent state. We return to this point below.

The dynamical behavior in the regions identified by the linear stability theory is the subject of the following section.

\section{Numerical results}

In this section we study the dynamics of Eqs. (26)-(28) in the intervals of $\mu$ identified in the preceding section. We consider only the four cases shown in Fig. 2, hereafter referred to as Cases I through IV.

\subsection{Case I: Periodic oscillations}

Case I is the simplest and exhibits periodic oscillations only, albeit of two distinct types. In the region of interest identified in Section 3 we find stable $R_{1}$-symmetric periodic orbits, labeled $R_{1 \mathrm{~s}}$ in Fig. 3a. These disappear with increasing $\mu$ at a Hopf bifurcation on the $P_{+}$branch, and annihilate with unstable $R_{1}$-symmetric solutions (labeled $R_{1 \mathrm{u}}$ ) at a saddle-node bifurcation $\mathrm{SN}$ as $\mu$ decreases (see Fig. 3a). The latter are created in a gluing bifurcation at $\mu=$ $\mu_{\mathrm{g}} \approx 1.926$ involving an unstable $P_{+}$state and a pair of unstable asy mmetric periodic orbits (labeled $A_{\mathrm{u}}$ ) produced in a (subcritical) Hopf bifurcation on the branch of mixed modes $M$. Thus, the asymmetric periodic orbits are unstable throughout their range of existence, $\mu_{\mathrm{g}}<\mu<\mu_{H} \approx 1.932$. The gluing bifurcation is shown in two different projections in Fig. 3b. The eigenvalues of the fixed point $P_{+}$at $\mu=\mu_{\mathrm{g}}$ are $0.16,-0.004,-1.1 \pm 1.9 \mathrm{i}$ and -2.16 ; the eigenvectors of the first two of these are $(\operatorname{Re}(X), \operatorname{Im}(X), \operatorname{Re}(Y), \operatorname{Im}(Y), v)=(0.8093,0.5869,0,0,0.0237)$ and $(0.6392,0.4902,0,0,0.592)$. Thus, both eigenvectors are odd with respect to $R_{1}$, and the homoclinic bifurcation at $\mu=\mu_{\mathrm{gg}}$ is of type $a$ in the terminology of Glendinning [15], with no stable periodic orbits or complex dynamics near $\mu_{\mathrm{g}}$. The saddle-node bifurcation on the $R_{1}$-symmetric branch is nonlocal and consistent with this classification. 
(a)

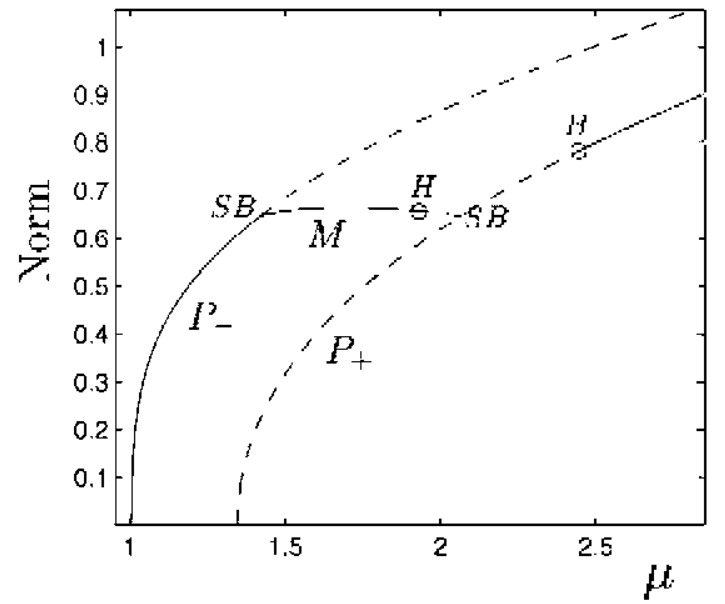

(c)

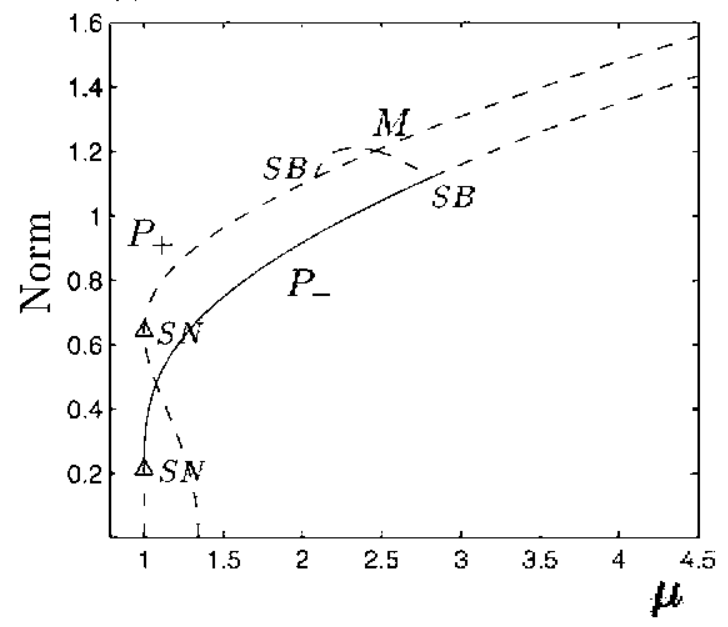

(b)

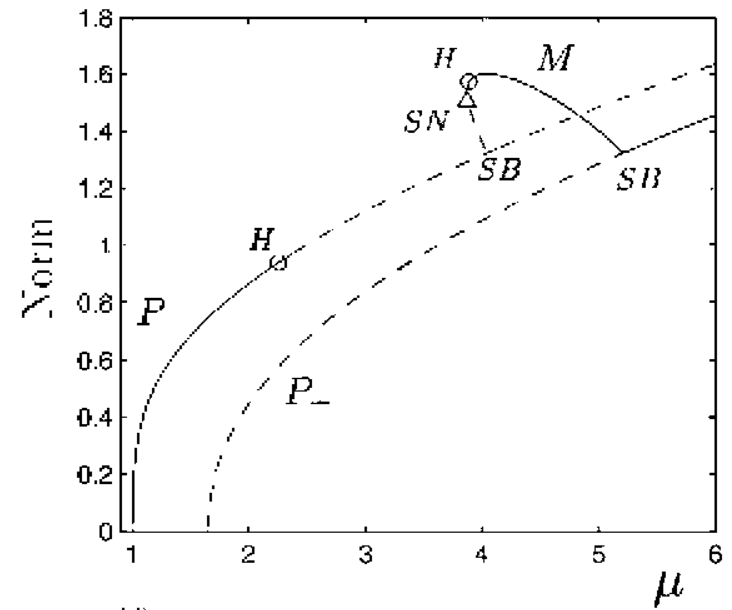

(d)

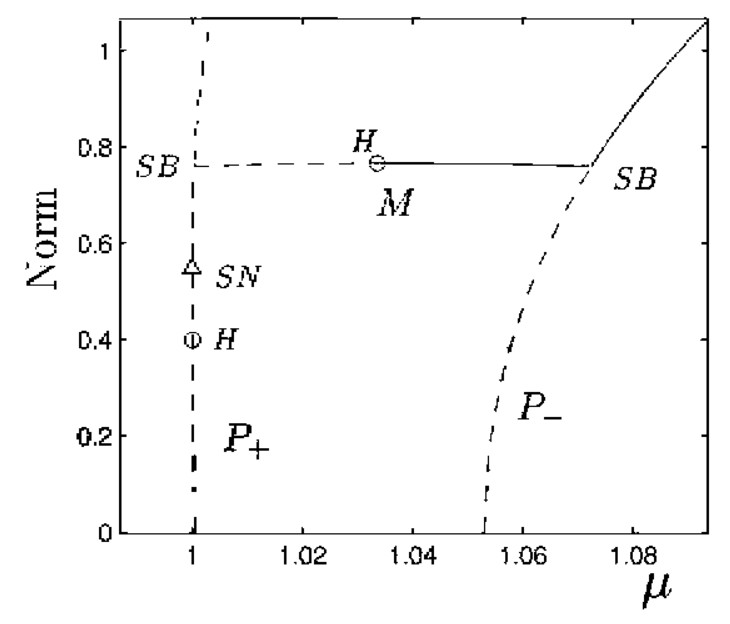

Fig. 2. Bifurcation diagrams for the pure modes $P_{-}$and mixed modes $M$ showing the Euclidean norm $\|(X, Y, v)\| \equiv \sqrt{ }|X|^{2}+|Y|^{2}+v^{2}$ as a function of $\mu$. Solid (broken) lines indicate stable (unstable) steady states. Saddle-node $(\triangle)$, symmetry-breaking and Hopf (o) bifurcations are labeled by SN, SB and $H$, respectively. 'The parameters are: (a) $I^{\circ}=0.5, \Lambda=-0.4, \alpha_{1}=0.4, \alpha_{2}=-2.58, \gamma=0.2,8=0.01$; (b) $I^{\prime}=0.7$, $\Lambda=-0.6, \alpha_{1}=0.4, \alpha_{2}=-2.58, \gamma=-0.2, \varepsilon=0.01$; (c) $\Gamma=-0.5, \Lambda=0.4, \alpha_{1}=0.4, \alpha_{2}=-2.58, \gamma=-0.6, \varepsilon=0.01 ;$ (d) $\Gamma=0.15$, $\Lambda=0.18, \alpha_{1}=0.48, \alpha_{2}=-0.58, \gamma=-0.35, \varepsilon=0.001$.

\subsection{Case II: Relaxation oxcillations and canards}

Case II is very interesting from a theoretical point of view, even though no chaotic dynamics appear to be present. As shown in Fig. 4a, the periodic orbits in the system undergo two distinct types of transition as $\mu$ increases. In the first, near $\mu=2.7$, a pair of $R_{2}$-symmetric periodic orbits, created in a Hopf bifurcation on the $P_{-}$branch, forms a pair of heteroclinic connections between $P_{+}$and $-P_{+}$. Fig. 5a shows one of the $R_{2}$-symmetric periodic orbits, in two different projections, just before this transition, while Fig. $5 \mathrm{~b}$ shows one of the two periodic orbits that result from it. Each of these orbits has $R_{1} R_{2}$-symmetry. Such symmetry-switching bifurcations are typical of $D_{2}$-symmetric systems [38,21] and occur as a result of the formation and breakup of a pair of heteroclinic connections. Since the eigenvalues of $P_{+}$at the bifurcation are $0.2770,-0.0182,-2.2687$ and $-1 \pm 3.3415 \mathrm{i}$ 
(a)

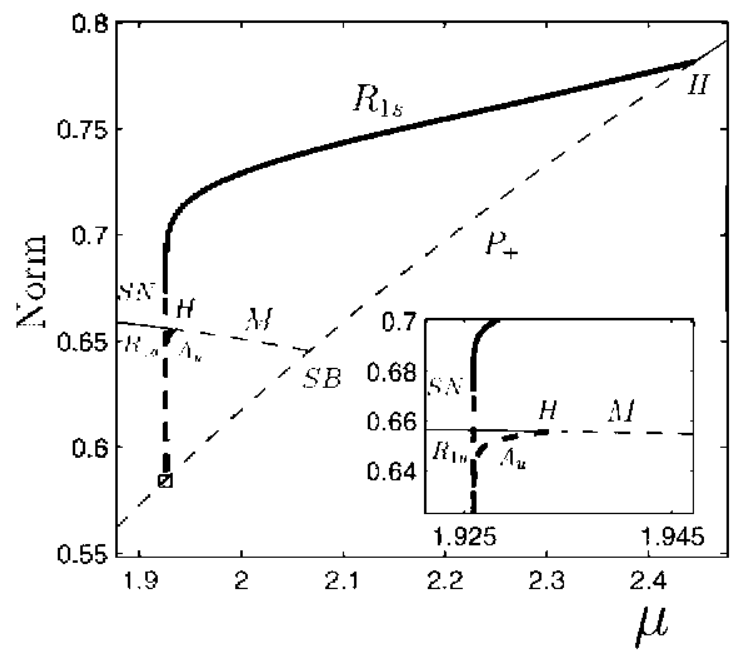

(b)
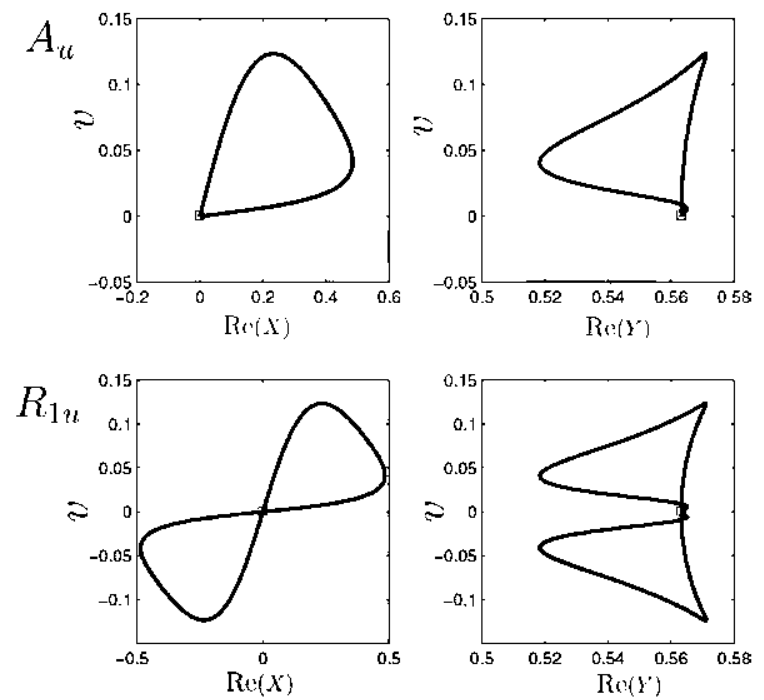

Fig. 3. Case I, corresponding to Fig. 2a. (a) Branches of steady states and periodic orbits in terms of the Euclidean nom $\|(X, Y, v)\|$ and the $L_{2}$ norm $\|(X, Y, v)\|_{L_{2}}$, respeetively, as a function of $\mu$. Thick solid (dashed) lines indicate stable (unstable) periodic orbits generated in Hopf bifurcations $(H)$ on $P_{+}$and $M$. 'Thin solid (dashed) lines indicate stable (unstable) steady states. (b) Two projections of the unstable periodic orbits close to the glung bifurcation at $\mu=\mu_{\mathrm{g}}$ an unstable asymmetric periodic orbit $A_{\mathrm{u}}$ and an unstable $R_{1}$-symmetric periodic orbit $R_{1 \mathrm{u}}$. The symbol - indicates the location on $P_{+}$of the global bifurcation.

with $(\operatorname{Re}(X), \operatorname{Im}(X), \operatorname{Re}(Y), \operatorname{Im}(Y), v)=(-0.7808,-0.6243,0,0,0.0242)$ and $(0.5416,0.4885,0,0,0.6842)$ as the first two eigenvectors the heteroclinic cycle $P_{+} \leftrightarrow-P_{+}$is asymptotically planar, with no stable periodic orbits or complex dynamics nearby. Consequently the two stable orbits must go through saddle-node bifurcations before the switching bifurcation can take place, cf. [15].
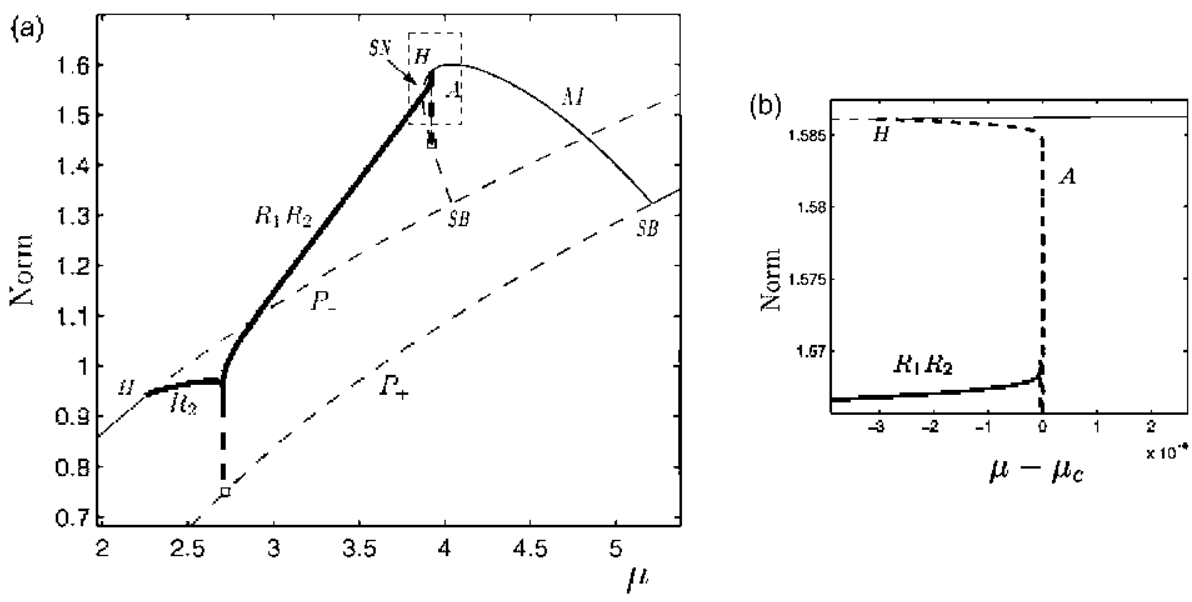

Fig. 4. Case II. (a) Branches of steady states and periodic orbits, in tems of the Euclidean norm $\|(X, Y, v)\|$ and the $L_{2}$ nonn $\|(X, Y, v)\| L_{2}$, respectively, as a function of $\mu$. Global bifurcations are indicated by open squares, while thick solid (dashed) lines correspond to stable (unstable) periodic orbits, and thin solid (dashed) lines correspond to stable (unstable) steady states. (b) Enlargement of the framed region in (a) centered at $\mu_{c}=3.9210403$, indicating the location of the $R_{1} R_{2}$-symmetric and asymmetric (A) orbits. Remaining parameters are as in Fig. $2 \mathrm{~b}$. 

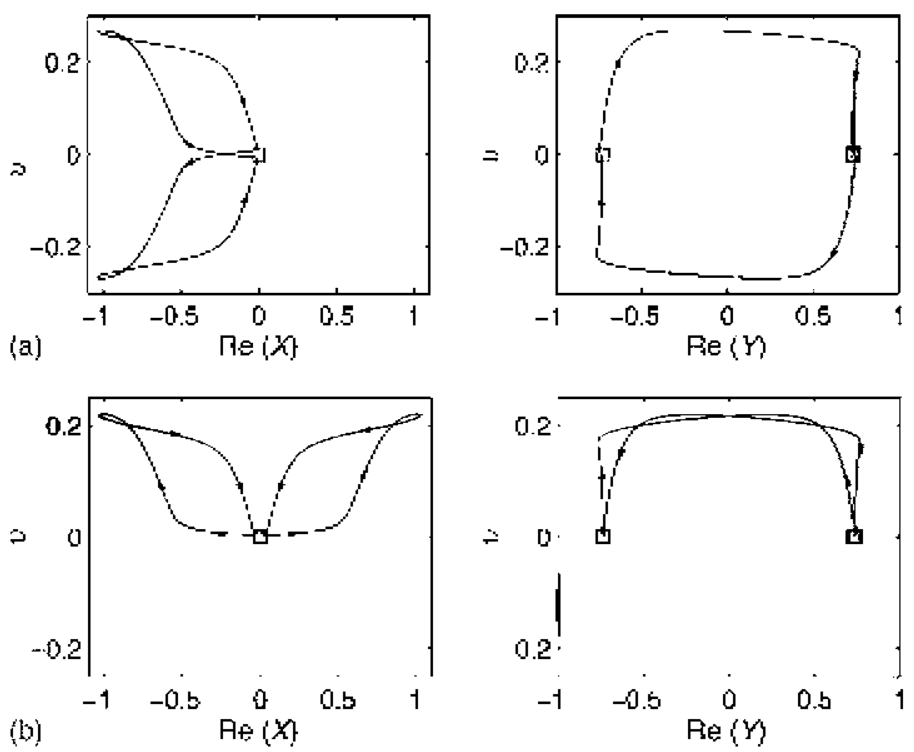

Fig. 5. Case II. A symmetry-switching transition involving the pure modes $\pm P_{+}$, in terms of projections onto the $(\operatorname{Re}(X), v)$ and $(\operatorname{Re}(Y), v)$ variables. (a) $\Lambda$ periodic $R_{2}$-symmetric orbit at $\mu=2.704115$. (b) $\Lambda$ periodic $R_{1} R_{2}$-symmetric orbit at $\mu=2.704122$. The symbol - indicates the location of the unstable fixed points $\pm P_{1}$. The parameters correspond to those of $\mathrm{Fig} .2 \mathrm{~b}$.

The second transition, near $\mu \approx 3.9210403$, is of much greater interest. Here each of the stable $R_{1} R_{2}$-symmetric periodic orbits created in the first transition (Fig. 4a) appears to "break up" in what looks like a saddle-node bifurcation into a pair of unstable periodic orbits with no symmetry, which in turn disappear in a subcritical Hopf bifurcation on the branch of mixed modes at $\mu_{H}=3.92073453$ (Fig. 4b). Fig. 6 shows these two orbits near this transition, and illustrates the abrupt change in the oscillation amplitude that results from it. Numerically, we find that the period of
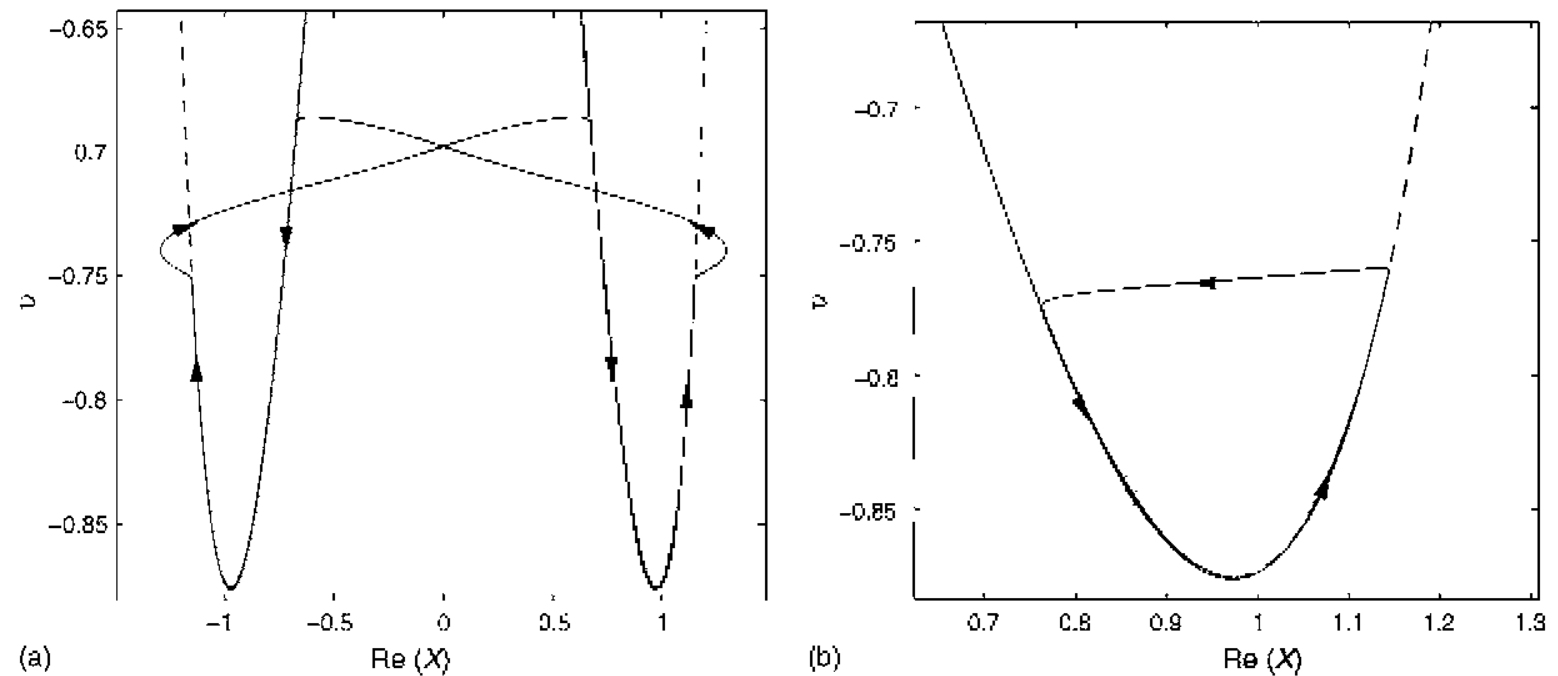

Fig. 6. Case II. Periodic orbits close to the codimension-two global bifurcation at $\varepsilon=0, \mu=\mu_{\mathrm{c}}$, superposed on the mullcline $\Sigma$. (a) $\Lambda$ stable $R_{\mid} R_{2}$-symmetric periodic orbit at $\mu=3.921039743, \varepsilon=0.01$. (b) An unstable asymmetric periodic orbit at $\mu=3.921040287, \varepsilon=0.01$. The remaining parameters are as in Fig. $2 \mathrm{~b}$. 
both types of oscillation appears to diverge at a common value of $\mu$ near $\mu=3.9210403$, hereafter $\mu_{\mathrm{o}}$. However, the nature of this divergence is highly unusual, and does not conform with the logarithmic divergence associated with an approach to a global bifurcation, even though Fig. 4a suggests that the transition between the small and large amplitude oscillations is somehow related to the proximity to an unstable mixed mode. It is clear, moreover, that the transition cannot be a gluing bifurcation of the ty pe encountered in Case I, since any gluing bifurcation between a pair of asymmetric orbits and an $R_{1} R_{2}$-symmetric periodic orbit at an asymmetric mixed mode is necessarily of codimension two, and hence nongeneric in one parameter families of vector fields.

With $\varepsilon=0.01$ it is exceedingly difficult to unravel the details of this unusual transition by numerical computation alone. We have therefore adopted a dual approach, by examining first the limit $\varepsilon \rightarrow 0$, and then performing detailed computations for somewhat larger values of $\varepsilon$ to confirm the predictions of the $\varepsilon \rightarrow 0$ analysis. We begin by writing Eqs. (26)-(28) in the form

$$
\boldsymbol{X}^{\prime}=\boldsymbol{F}_{1}(\boldsymbol{X}, \boldsymbol{Y}, v ; \mu), \quad \boldsymbol{Y}^{\prime}=\boldsymbol{F}_{2}(\boldsymbol{X}, \boldsymbol{Y}, v ; \mu), \quad v^{\prime}=\varepsilon G(\boldsymbol{X}, \boldsymbol{Y}, v),
$$

where $X=(\operatorname{Re}(X), \operatorname{Im}(X)), Y=(\operatorname{Re}(Y), \operatorname{Im}(Y))$ and suppose that $0<\varepsilon \ll 1$. In this regime, Eqs. (42) exhibit relaxation oscillations, and we can understand the properties of these oscillations by examining first the case $\varepsilon=0$. In this case, $v$ becomes a parameter, and Eqs. (42) become

$$
\boldsymbol{X}^{\prime}=\boldsymbol{F}_{1}(\boldsymbol{X}, \boldsymbol{Y} ; v, \mu), \quad \boldsymbol{Y}^{\prime}=\boldsymbol{F}_{2}(\boldsymbol{X}, \boldsymbol{Y} ; v, \mu) .
$$

This pair of equations can have both steady state solutions and periodic solutions. Of particular significance is the one-dimensional nullcline $\Sigma: \boldsymbol{F}_{1}(\boldsymbol{X}, \boldsymbol{Y}, v ; \mu)=\boldsymbol{F}_{2}(\boldsymbol{X}, \boldsymbol{Y}, v ; \mu)=0$ that contains the steady states when $\varepsilon>0$ and consists of them when $\varepsilon=0$. These may be stable or unstable. When $0<\varepsilon \ll 1$ the solutions of Eqs. (42) can be thought of as drifting slowly along the attracting part of $\Sigma$. Typically this slow drift ends near folds on $\Sigma$ where the solution is forced away from $\Sigma$ and the slow drift is replaced by a fast transition that takes the system to another branch of $\Sigma$. This is the essence of all relaxation oscillations. However, in certain circumstances the periodic oscillations may follow the unstable part of $\Sigma$ after the fold. This is the hallmark of the so-called canard phenomenon, and related behavior has been seen, for example, in model equations describing chemical systems $[8,35]$. Like the present system these systems also exhibit abrupt transitions from small amplitude oscillations to large amplitude relaxation-1y pe oscillations, a behavior that has been called a canard explosion $[8,18,35]$.

To understand this behavior in the present context we show in Fig. 7a, a projection of $\Sigma$ on the $(\operatorname{Re}(X), v)$ plane when $\mu \approx \mu_{I I}$. This projection shows four branches, $S^{ \pm}$(solid lines) and $U^{ \pm}$(dashed lines), corresponding respectively to stable and unstable steady states of Eqs. (43); the points $q_{ \pm}$denote saddle-node bifurcations where these steady states change their stability. Note that the branches $S^{+}$and $U^{+}$as well as the point $q^{+}$are related through $R_{1} R_{2}$-symmetry to $S^{-}, U^{-}$and $q^{-}$. Away from the fold points $q^{ \pm}$the manifolds $S^{ \pm}$and $U^{ \pm}$are normally hyperbolic. As a consequence when $0<\varepsilon \ll 1$ they perturb to normally hyperbolic invariant manifolds $M_{S}^{ \pm}$and $M_{U I}^{ \pm}$lying within $O(\varepsilon)$ of $S^{ \pm}$and $U^{ \pm}[13]$. The former is attracting, while the latter is of saddle-lype, properties that are inherited from those of $S^{ \pm}$and $U^{ \pm}$. Together these manifolds define the slow manifold of the system (42). To find the flow on this manifold due to the slow evolution of $v$ we solve (43) for $X, Y$ as functions of $v$ and compute $G(\boldsymbol{X}(v), \boldsymbol{Y}(v), v)$. The fixed points of this reduced flow with $v \neq 0$ approximate the mixed modes $M^{ \pm}$of (42) for $0<\varepsilon \ll 1$ and lie within $\Sigma$. Fig. $7 \mathrm{~b}$ shows these fixed points superposed on $\Sigma$ when $\varepsilon=0.01$. There are two pairs of such points, labeled $M_{1}^{ \pm}$and $M_{2}^{ \pm}$, of which $M_{1}^{ \pm} \approx q^{ \pm}$undergo a subcritical Hopf bifurcation at $\mu_{H}=3.92073453$. Numerically we find that the resulting unstable asy mmetric oscillations grow in amplitude as $\mu$ increases but remain unstable all the way to $\mu=\mu_{\mathrm{c}}$, where the transition to the stable large amplitude $R_{1} R_{2}$-symmetric oscillations takes place.

Fig. 8(a-d) describes geometrically what happens in a typical canard explosion. The figure is drawn for $0<\varepsilon \ll 1$ but does not distinguish between the slow unstable manifolds $M_{U}^{ \pm}$and the stable manifolds $W^{s}$ of the fixed points $M_{2}^{ \pm}$. This permits us to exiend $M_{U}^{ \pm}$past the folds $q^{ \pm}$. Fig. 8a shows the relative position of these manifolds prior to the explosion. The fixed point $M_{1}^{+} \in M_{S}^{+}$is attracting, while $M_{2}^{+} \in M_{U}^{+}$is a saddle. If $\mu$ is now increased $M_{1}^{+}$moves 

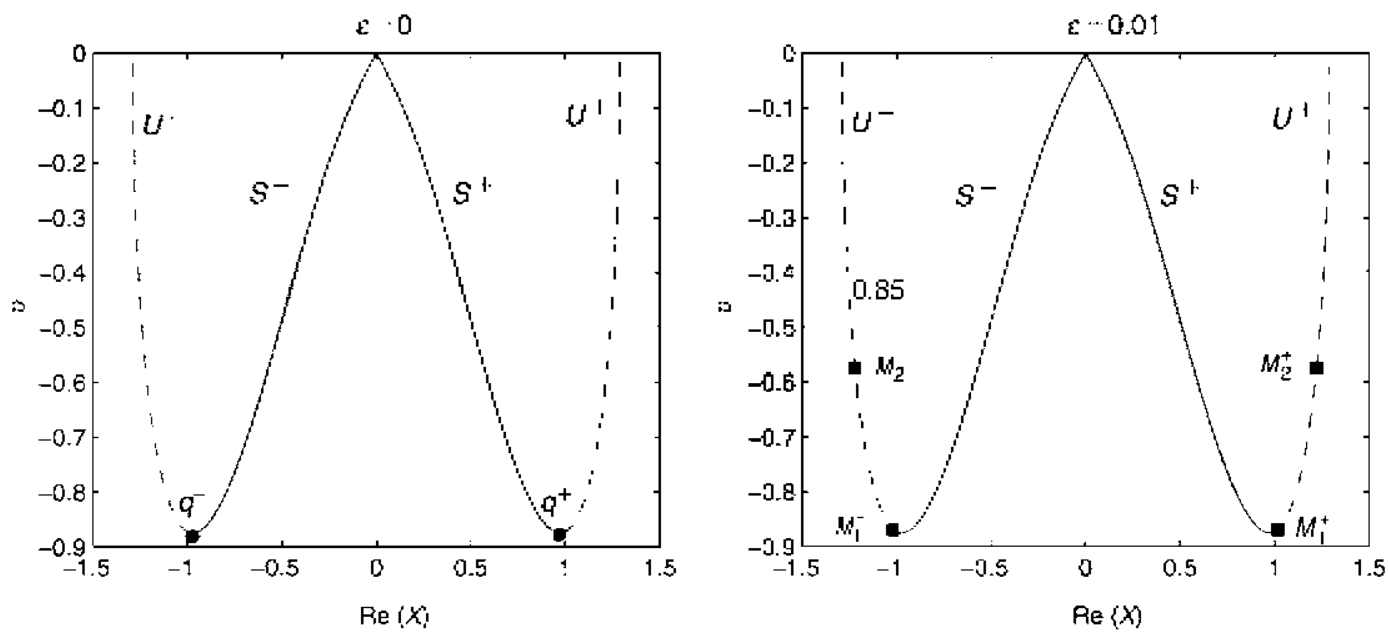

Fig. 7. Case II. Projection of the nulleline $\Sigma$ on $(\operatorname{Re}(X), v)$-plane for $\mu=3.9213453 \approx \mu_{I I}$ and the indicated values of $\varepsilon$. The remaining parameters are as in Fig. 2 h. Solid (dashed) line corresponds to stable (unstable) steady states of Kiqs. (42) for $\varepsilon=0$.

past $q^{+}$and falls in $M_{U}^{+}$, resulting in a Hopf bifurcation. Fig. 8b shows the relative orientation of $M_{S}^{+}$, the resulting small amplitude periodic solution $\gamma_{s}$ and $W^{8}\left(M_{2}^{+}\right)$when this bifurcation is supercritical. As $\mu$ increases further $M_{S}^{+}$and $W^{s}\left(M_{2}^{+}\right)$approach one another, and at a critical value $\mu=\mu_{\mathrm{c}}$ they may coalesce (see Fig. 8c). As this happens the small amplitude oscillation grows and at $\mu=\mu_{\mathrm{c}}$ it collides with $M_{2}^{+}$, forming a homoclinic connection. Beyond this critical parameter value the homoclinic connection breaks, forming a large amplitude periodic orbit $\gamma_{\ell}$ lying outside of $W^{\mathrm{s}}\left(M_{2}^{+}\right)$(Fig. 8d). Here the formation of a canard therefore corresponds to the appearance of a homoclinic connection and hence is associated with a global bifurcation [35].

In our case, however, the Hopf bifurcation is subcritical, $M_{1}^{+}$is stable, and the details of the transition differ $[2,3]$. Fig. 8e and $\mathrm{f}$ shows the analogues of Fig. $8 \mathrm{~b}$ and $\mathrm{d}$ for this case. Prior to the canard the small amplitude periodic solution $\gamma_{\mathrm{s}}$ is unstable (Fig. 8e) and coexists with a stable large amplitude periodic orbit $\gamma_{\ell}$ lying outside of $W^{\mathrm{s}}\left(M_{2}^{+}\right)$. As $\mu$ approaches $\mu_{\mathrm{c}}$ thesc orbits both approach $M_{2}^{+}$along $W^{\mathrm{s}}\left(M_{2}^{+}\right)$and at $\mu=\mu_{\mathrm{c}}$ (Fig. 8c) $\gamma_{\mathrm{s}}$ appears to coincide with a part of $\gamma_{\ell}$. Once $\mu>\mu_{\mathrm{c}}$ the slow manifold $M_{S}^{+}$lies on the inside of $W^{\mathrm{s}}\left(M_{2}^{+}\right)$and no oscillations are present (Fig. 8f). Thus, the small amplitude unstable oscillation annihilates the large amplitude stable oscillation, a transition that must take place via the formation of a comection to $M_{2}^{+}$since the two oscillations have different symmetry properties. Thus, the canard at $\mu=\mu_{\mathrm{c}}$ looks like a saddle-node bifurcation of periodic orbits (cr. [8]) but is in fact also associated with a global bifurcation.

When $\varepsilon$ is finite but sufficiently small $(\varepsilon=0.01$ is sufficiently small in this sense) the behavior of the system (26)-(28) is indistinguishable from the qualitative predictions based on the above picture. Fig. 6 shows an unstable asymmetric small amplitude oscillation at $\mu=3.921040287$ and a stable $R_{1} R_{2}$-symmetric relaxation oscillation at $\mu=3.921039743$, each projected on the $(\operatorname{Re}(X), v)$ variables and superposed on the nullcline $\Sigma$, for $\varepsilon=0.01$. In cach casc, the arrows indicate the direction of motion for comparison with Fig. $8 \mathrm{e}$ and $\mathrm{f}$. The figure confirms that the slow manifold for $\varepsilon=0.01$ does indeed follow the locus of fixed points computed with $\varepsilon=0$ (Fig. 7a), and that as $\mu$ increases a pair of unstable small amplitude asymmetric periodic orbits is born in a (subcritical) Hopf bifurcation of $M_{1}^{ \pm} \approx q^{ \pm}$. As $\mu$ increases further the amplitude of each of these grows, initially slowly but then more rapidly, prior to the canard transition at $\mu=\mu_{\mathrm{c}}$. At $\mu_{\mathrm{c}}$ the unstable periodic orbil $\gamma_{\mathrm{s}}^{+}$follows $M_{U}^{+}$, the unstable part of the slow manifold between $M_{1}^{+}$and the saddle-ty pe fixed point $M_{2}^{+}$(Fig. 6b), forming a saddle-loop connection. Fig. 9 shows that during this process the period of both the asymmetric and the $R_{1} R_{2}$-symmetric oscillations diverges, confirming 

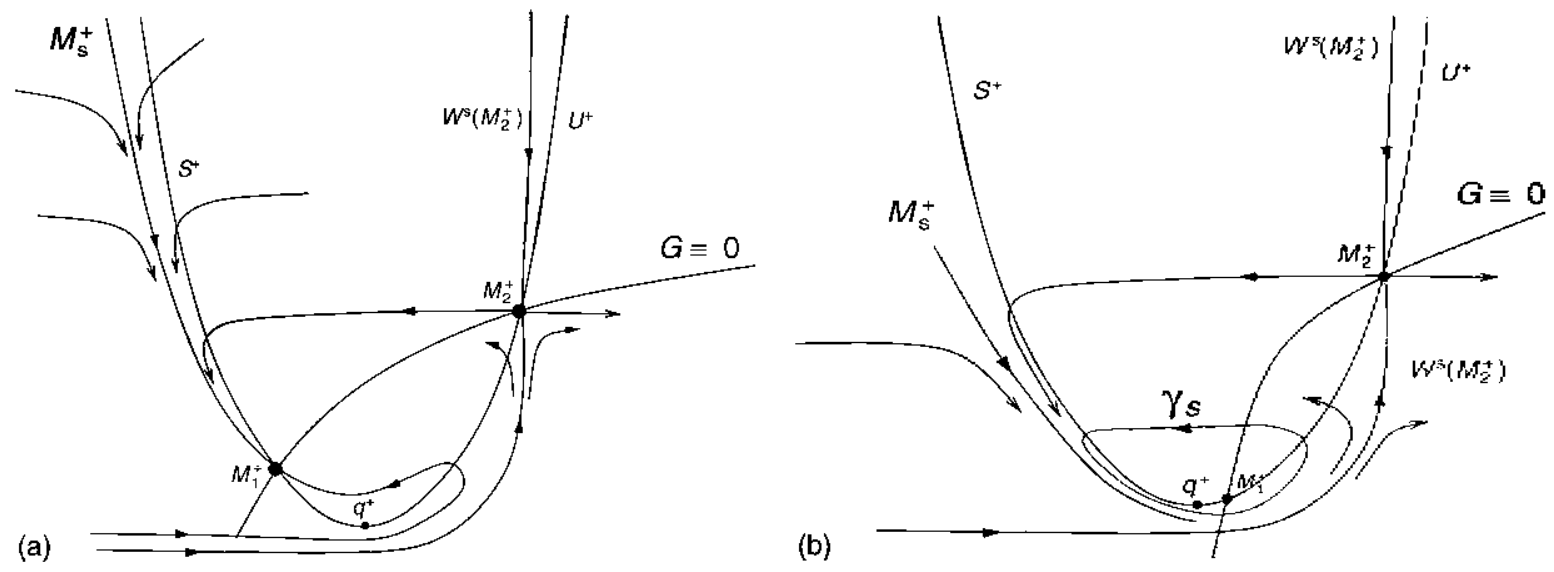

(c)

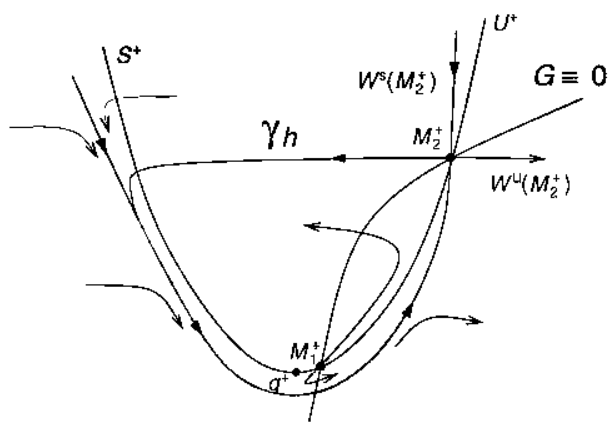

(d)
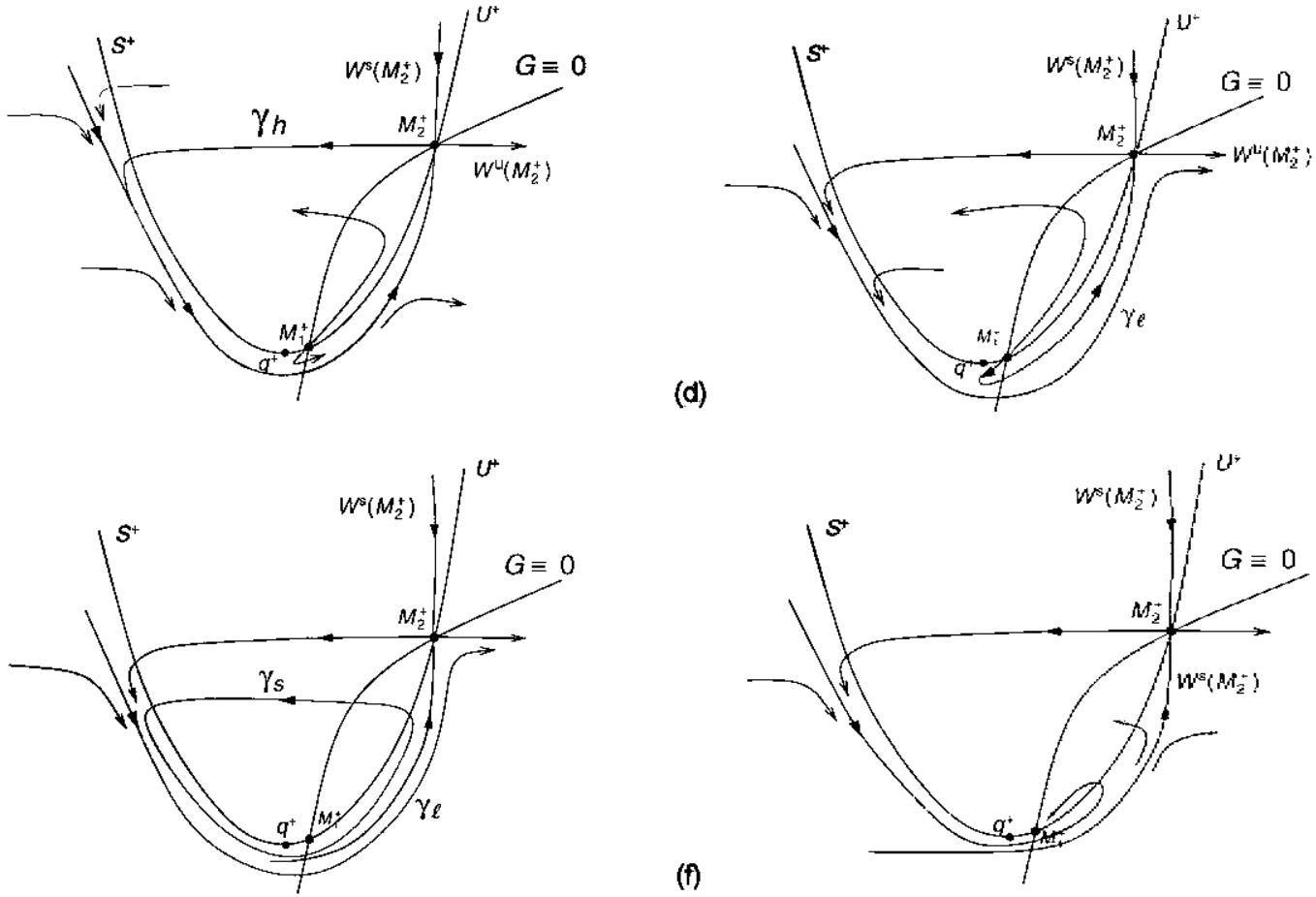

(e)

(f)

Fig. 8. Case II. Projection of the nullclines $\Sigma$ and $V$ on the $(\operatorname{Re}(X), v)$-plane, showing the relative orientation of the slow manifold $M_{S}^{+}$and the stable and unstable manifolds $W^{s, l}$ of the fixed point $M_{2}^{+}$. (a) $M_{1}^{-}$is stable. (b) $M_{1}^{+}$undergoes a supereritical Hopf bifurcation at $\mu=\mu_{H}$ producing a stable small amplitude asymmetric orbil $\gamma_{\mathrm{s}}$. (c) The canard manifold $W^{\mathrm{s}}\left(M_{2}^{\dagger}\right)$ at $\mu=\mu_{\mathrm{c}}$, conmecting $M_{S}^{!}$with $M_{V}^{!}$, showing the homoclinic comnection $\gamma_{\mathrm{h}}$. (d) The formation of a stable large amplitude relaxation oscillation $\gamma_{f}$, when $\mu>\mu_{\mathrm{c}}$. (e) $M_{1}^{+}$undergoes a subcritical Hopf bifurcation at $\mu=\mu_{H}$ producing an unstable small amplitude asymmetric orbit $\gamma_{s}$ within a stable large amplitude relaxation oscillation $\gamma_{f}$. (f) The formation of a canard at $\mu=\mu_{\mathrm{c}}$ is responsible for the absence of both periodic orbits when $\mu>\mu_{\mathrm{c}}$. 


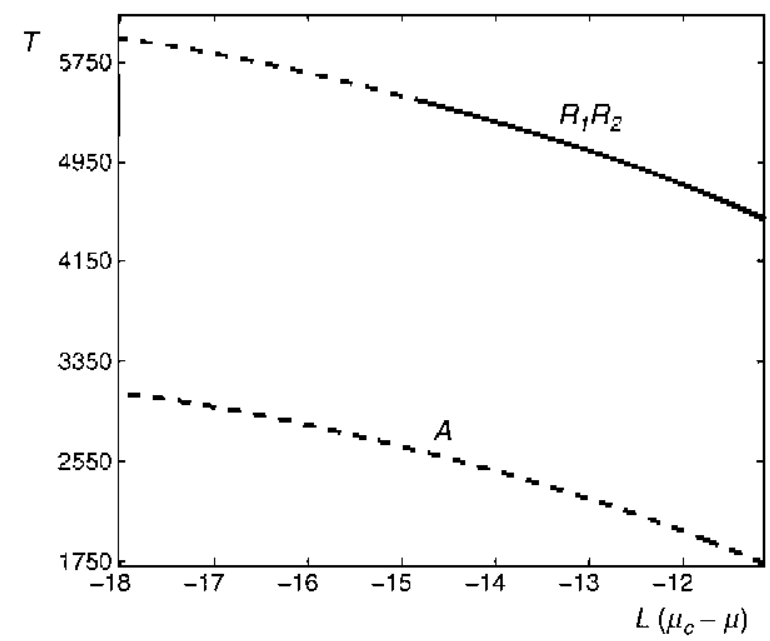

Fig. 9. Case II. The period $\%$ of asymmetric and $k_{1} R_{2}$-symmetric oseillations near the canard at $\mu=\mu_{0} \approx 3.9210403$. 'The symbol $h$ indicates the natural logarithm. Solid (broken) lines indicate stable (unstable) oscillations. Both periods diverge as $\mu \rightarrow \mu_{c}-$, indicating proximity to a global bifurcation.

the presence of a global bifurcation involving $M_{2}^{+}$, and that for both oscillations this occurs from the same side. However, despite much effort (see Fig. 9) we have been unable to confirm the expected result that the oscillation period $T$ of both the small and large amplitude oscillations should diverge logarithmically, as $T \sim-\lambda_{\mathrm{u}}^{-1} \ln \left|\mu-\mu_{\mathrm{c}}\right|$, where $\lambda_{\mathrm{u}}$ is the unstable cigenvalue of $M_{2}^{+}$.

These and other conflicts with expectation are all reconciled when one recognizes that the location of the detachment of the trajectory from the unstable slow manifold is exponentially sensitive to the value of $\varepsilon$ and that the range of $\mu$ around $\mu_{\mathrm{e}}$ during which canards are present is proportional to $\mathrm{exp}-c / \varepsilon$, where $c$ is a positive constant $[11,18]$. This fact suggests in turn that there is an exponentially small neighborhood of $\mu_{\mathrm{c}}$ in which the finite value of $\varepsilon$ restores the expected behavior, such as the logarithmic divergence. In fact, we find that the finite valuc of $\varepsilon$ 'unfolds' the saddle-node/global bifurcation present at $\varepsilon=0$ (cf. [16]), with the result that the global bifurcation splits into two successive codimension-one global bifurcations, one involving the small amplitude asymmetric oscillations and the other the large amplitude $R_{1} R_{2}$-symmetric oscillations, with the saddle-node bifurcation now occurring on one or other of these oscillation branches. The behavior near each of these global bifurcations is determined by the cigenvalue ratio $\delta=\left|\lambda_{\mathrm{s}} / \lambda_{\mathrm{u}}\right|$, where $\lambda_{\mathrm{s}} \approx-0.00\left(051\right.$ is the least stable cigenvalue of $M_{2}^{+}$and $\lambda_{\mathrm{u}} \approx 0.4889$ is its unstable eigenvalue, both computed at $\mu=\mu_{\mathrm{c}}$ for $\varepsilon=0.01$. Thus, $\delta \ll 1$. Under these conditions classical theory [45] shows that neither type of periodic orbit will be stable near $\mu=\mu_{\mathrm{o}}$. It follows therefore that once $0<\varepsilon \ll 1$ the saddle-node bifurcation must move away from $\mu=\mu_{\mathrm{e}}$, and that it is the stable $R_{1} R_{2}$-symmetric orbit that must undergo a saddle-node bifurcation prior to the formation of the canard at $\mu=\mu_{\mathrm{c}}$. If this is so $\mu_{\mathrm{c}} \geq 3.921040287$, and the two periodic orbits approach their individual global bifurcations from opposite sides after all.

In order to confirm these conclusions we have recomputed our results with $\varepsilon=0.2$. With this larger value of $\varepsilon$ the qualitative picture remains unchanged (Fig. 10a) but the nature of the transition becomes much clearer. Fig. 10b shows that the asymmetric oscillations continue to be subcritical and unstable, and that these approach monotonically a global bifurcation at $\mu_{\mathrm{a}}=3.881857$ involving the mixed modes $M_{2}^{ \pm}$as $\mu$ increases. Moreover, this bifurcation is now distinct from the corresponding global bifurcation on the branch of $R_{1} R_{2}$-symmetric relaxation oscillations which takes place at a larger value of $\mu, \mu_{\mathrm{s}}=3.882094$. In addition, one can now follow the oscillation period close to cach global bifurcation, and finds that in both cases it diverges logarithmically, as expected from global bifurcations. Note also that the $R_{1} R_{2}$-symmetric oscillations remain stable almost until the end of the branch, but that right before the end they go through a saddle-node bifurcation at which they lose stability. Thus, on both 

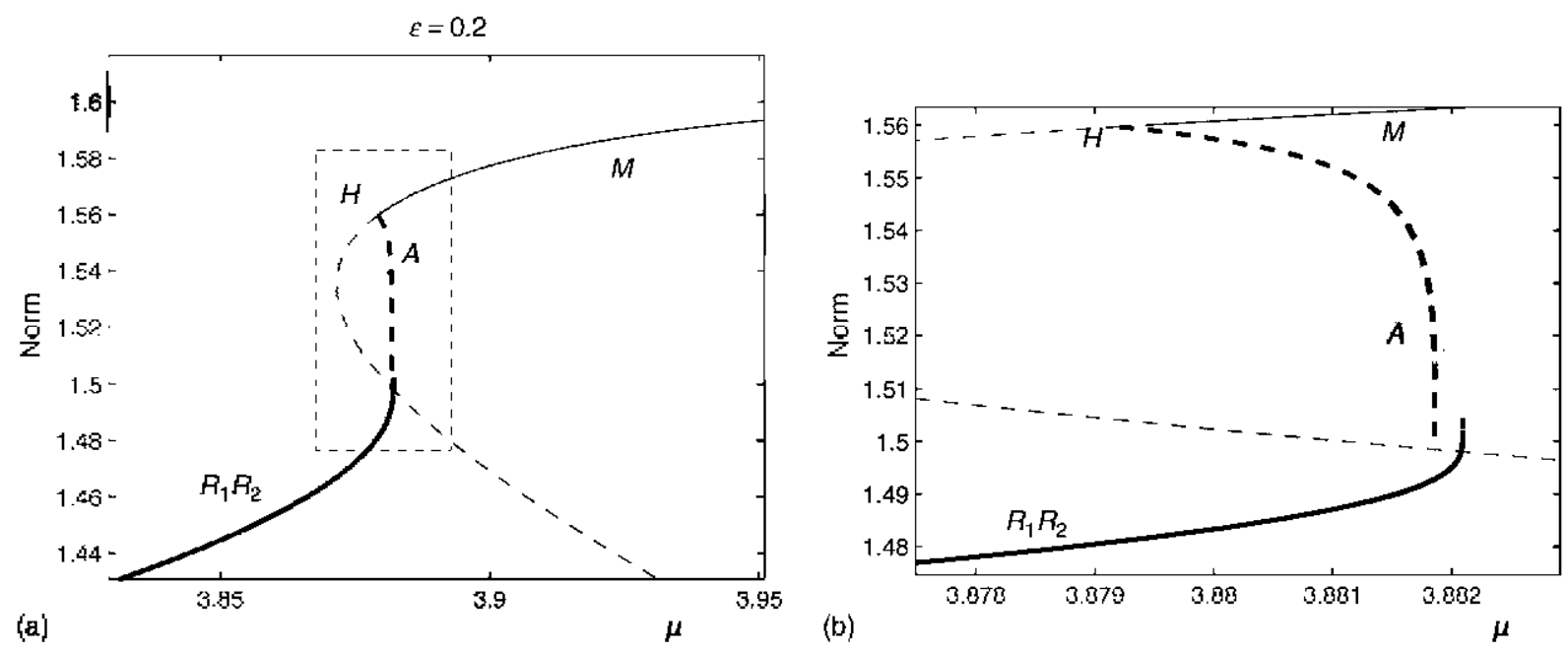

Fig. 10. (a) Bifurcation diagram for the same parameters as Case [l but with $\varepsilon=0.2$ for comparison with Fig. 4a. (b) 1)etail of the bifurcation diagram in (a) for comparison with Fig. $4 \mathrm{~h}$, showing the "unfolding' of the canard transition at finite $s$.

branches the oscillations are unstable near their respective global bifurcations, as expected from the fact that here too $\delta \ll 1$. We conclude that the strange behavior of the canard transition is a consequence of the exponentially small region in which the two global bifurcations and the accompanying saddle-node bifurcation occur. As a result the logarithmic divergence of the period is postponed to very high periods, the two families of periodic orbits undergo global bifurcations almost simultaneously, and the relaxation oscillations involved appear to approach the global bifurcation from the wrong direction and with the wrong stability assignment. It is reasonable to expect that for any finite value of $\varepsilon$ these 'paradoxes' are resolved in this cxponentially small region around $\mu=\mu_{\mathrm{c}}$ in the same way as found here for $\varepsilon=0.2$.

Finally, the theoretical prediction [18] that canards will form once $\mu-\mu_{H}=O(\varepsilon)$ is also broadly consistent with the numerical results. We believe, therefore, that except for the effects of symmetry the transition observed in Fig. 6 is essentially the result of a classical canard associated with a subcritical Hopf bifurcation.

\subsection{Case III: Symmetric and asymmetric bursting}

This case displays very interesting dynamics over a large (semi-infinite) range of the forcing amplitude $\mu$ where no stable steady states exist. As shown in Fig. 11, when the pure mode $P_{-}$loses stability in a symmetry-breaking bifurcation at $\mu=\mu \mathrm{SP} \approx 2.8$, small perturbations drive the system to a stable branch of $R_{1} R_{2}$-symmetric periodic orbits. If this periodic branch is continued backwards (i.e., for $\mu<\mu_{\mathrm{SB}}$ ) one finds that it ultimately terminates in a hetcroclinic bifurcation involving the nonsy mmetric fixed points $M^{ \pm}$. The cigenvalues of $M^{ \pm}$at this bifurcation are $0.001940,-0.42229,-0.998 \pm 4.819 \mathrm{i}$ and -1.58926 and hence no chaotic dynamics result. An entirely different scenario unfolds when this periodic branch is continued for $\mu>\mu_{\mathrm{SB}}$. First, the $R_{1} R_{2}$-symmetric branch undergoes a pitchfork bifurcation that generates stable asy mmetric periodic orbits (see Fig. 11 , inset). With further increase in $\mu$ these asymmetric orbits undergo a period doubling bifurcation PD and, as shown in the bifurcation diagram of Fig. 12, chaotic dy namics are found not long after. The chaotic behavior is marked by a crisis in which two asymmetric chaotic attractors collide at $\mu \approx 4.903$ and merge, forming a symmetric chaotic attractor (a symmetry-increasing bifurcation). The interval over which chaos is observed is relatively short $(4.9() 2 \lesssim \mu \lesssim 4.92)$, however, and the system is soon attracted to a new branch of $R_{1} R_{2}$-symmetric periodic orbits created in a saddle-node bifurcation. When, in turn, this $R_{1} R_{2}$-symmetric branch loses stability, we observe a new branch of stable asymmetric periodic 


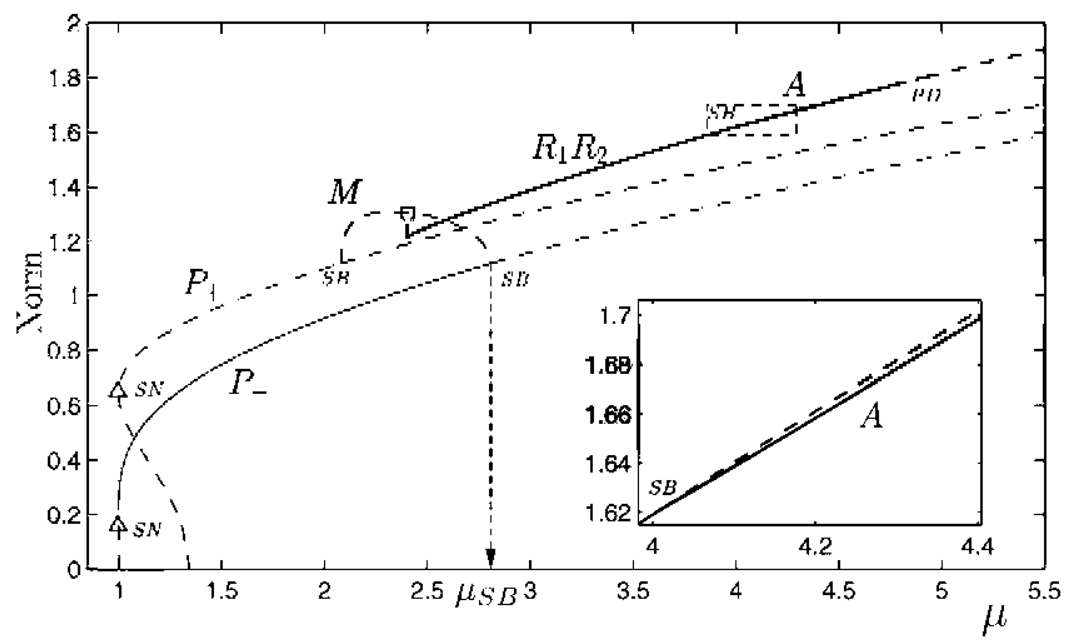

Fig. 11. Case III, corresponding to Fig. 2c. Bifurcation diagram for steady states and periodic orbits, in terms of the Euclidean norm $\|(X, Y, v)\|$ and the $L_{2}$ norm $|(X, Y, v)|_{L_{2}}$, respectively, as a function of $\mu$. 'Thick solid (dashed) lines correspond to stable (unstable) periodie orbits; thin solid (dashed) lines correspond to stable (unstable) steady states. 'The arrow indicates the loeation of a suberitical bifurcation on $P_{-}$to unstable $M$. This bifurcation produces a hysteretic transition to stable $R_{1} R_{2}$-symmetric oscillations that exist between the heteroclinic bifurcation indicaled by the symbol $\square$ and a symmetry-breaking bifurcation labeled SB (inset).

orbits created in a nearby saddle-node bifurcation. This sort of alternating transition between $R_{1} R_{2}$-symmetric and asymmetric oscillations is repeated again and again. Note also that the chaotic windows associated with these transitions become narrower and narrower as $\mu$ increases. In Fig. 13, we show stable attractors associated with three consecutive periodic windows in Fig. 12. These limit cycles are evidently relaxation oscillations, but this time involving slow drifts along branches of both equilibria and of periodic orbits, with fast jumps between them. Because of their resemblance to bursting in excitable membranes or spiking in neurons we refer to these oscillations as bursting or spiking as well. In our case, however, the bursting oscillations may be symmetric or asymmetric, with the symmetry alternately present and broken in successive periodic windows. In the following we suggest an explanation for this remarkable behavior adapted from ref. [41].

As in Case II the behavior described above can be understood, at least in part, by considering the nullcline $\Sigma: \boldsymbol{F}_{1}(\boldsymbol{X}, \boldsymbol{Y}, v ; \mu)=\boldsymbol{F}_{2}(\boldsymbol{X}, \boldsymbol{Y}, v ; \mu)=0$. As indicated in Fig. 14, the projection of $\Sigma$ onto the $(v, \operatorname{Re}(X))$ plane consists of pairs of branches of stable $\left(S^{ \pm}\right)$and unstable $\left(U^{ \pm}\right)$states, related by symmetry. However, in Case III Eqs. (42) with $\varepsilon=0$ also contain a one parameter family of attracting limit cycles. These periodic solutions are created, as $v$ increases, in a heteroclinic bifurcation involving the two fixed points $U^{+}$and $U^{-}$, and are $R_{1} R_{2}$-symmetric. Between this heteroclinic bifurcation and the fold points $q^{ \pm}$the stable $R_{1} R_{2}$-symmetric limit cycles cocxist with the stable fixed points $S^{ \pm}$(see Fig. 14). When $\varepsilon$ is finite but small these states couple to the slow evolution of the variable $v$, and the manifolds of stcady states and periodic orbits become part of the slow manifold of the system (42), cl. [41]. In the following we speak of the solutions as drifting along this manifold (the slow phase); this drift proceeds until the system is forced away from the slow manifold, heralding the onset of the fast phase of the oscillation that takes it back to the slow manifold. The plots in Figs. 13 can be interpreted in this light, with episodes of almost constant $\operatorname{Re}(X)$ corresponding to drift along the manifold of steady states $S^{ \pm}$and the return trajectory consisting of a drift along the branch of periodic orbits.

To understand the nature of the oscillations that result we begin by considering the drift along $M_{S}^{+}$, the part of the slow manifold near the stable steady states $S^{+}$. The drift is in the direction of increasing $v$ and so takes the system towards $q^{+}$(see Fig. 14). Near this point this slow drift ends and the system jumps abruptly to the branch of symmetric periodic states, labeled $p$. In Fig. 14, this transition is indicated by a vertical short-dashed line, and 


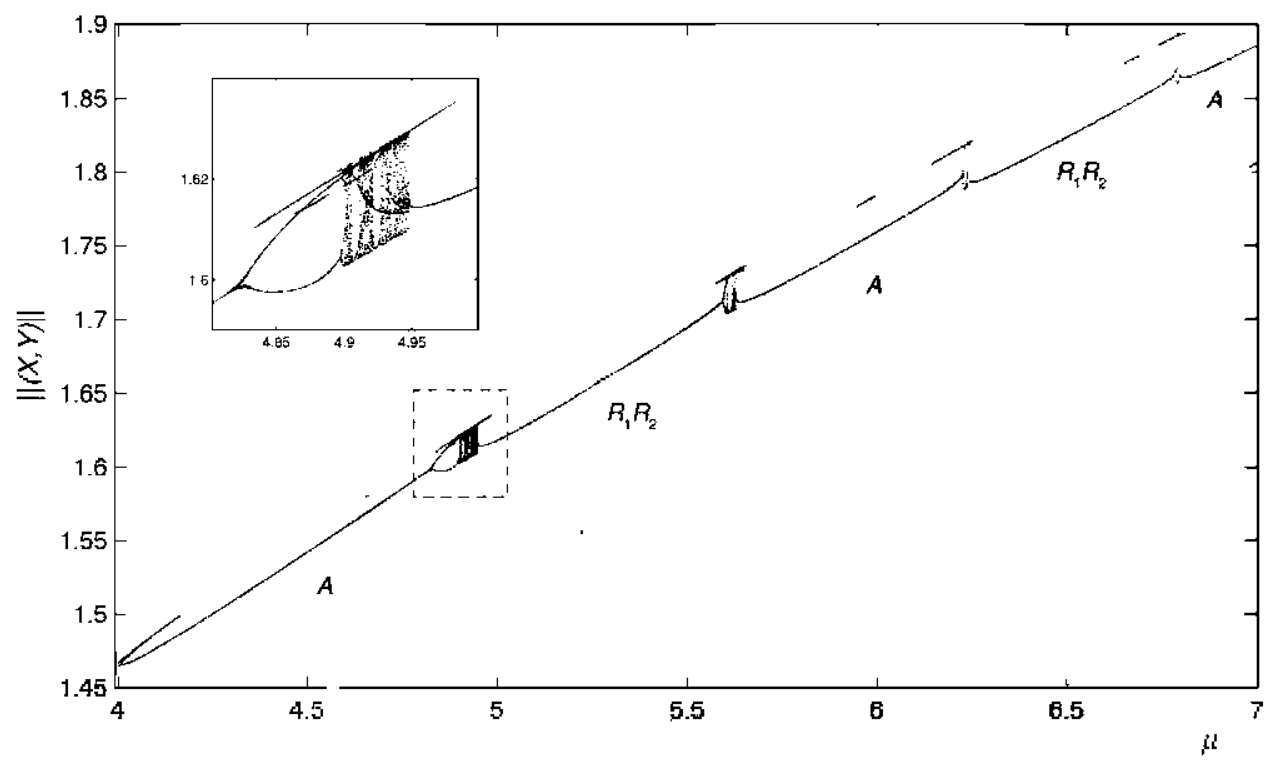

Fig. 12. Case III: bifurcation diagram showing an alternating sequence of $R_{1} R_{2}$-symmetric $\left(K_{1} R_{2}\right)$ and asymmetric $(A)$ periodic orbits. 'The figure is constructed by recording successive maxima of $\|(X, Y)\| \equiv \sqrt{\|X\|^{2}+\|Y\|^{2}}$ at each value of $\mu$. The inset shows an enlargement of the first chaotic region. The parameters are as in Fig. 2c.
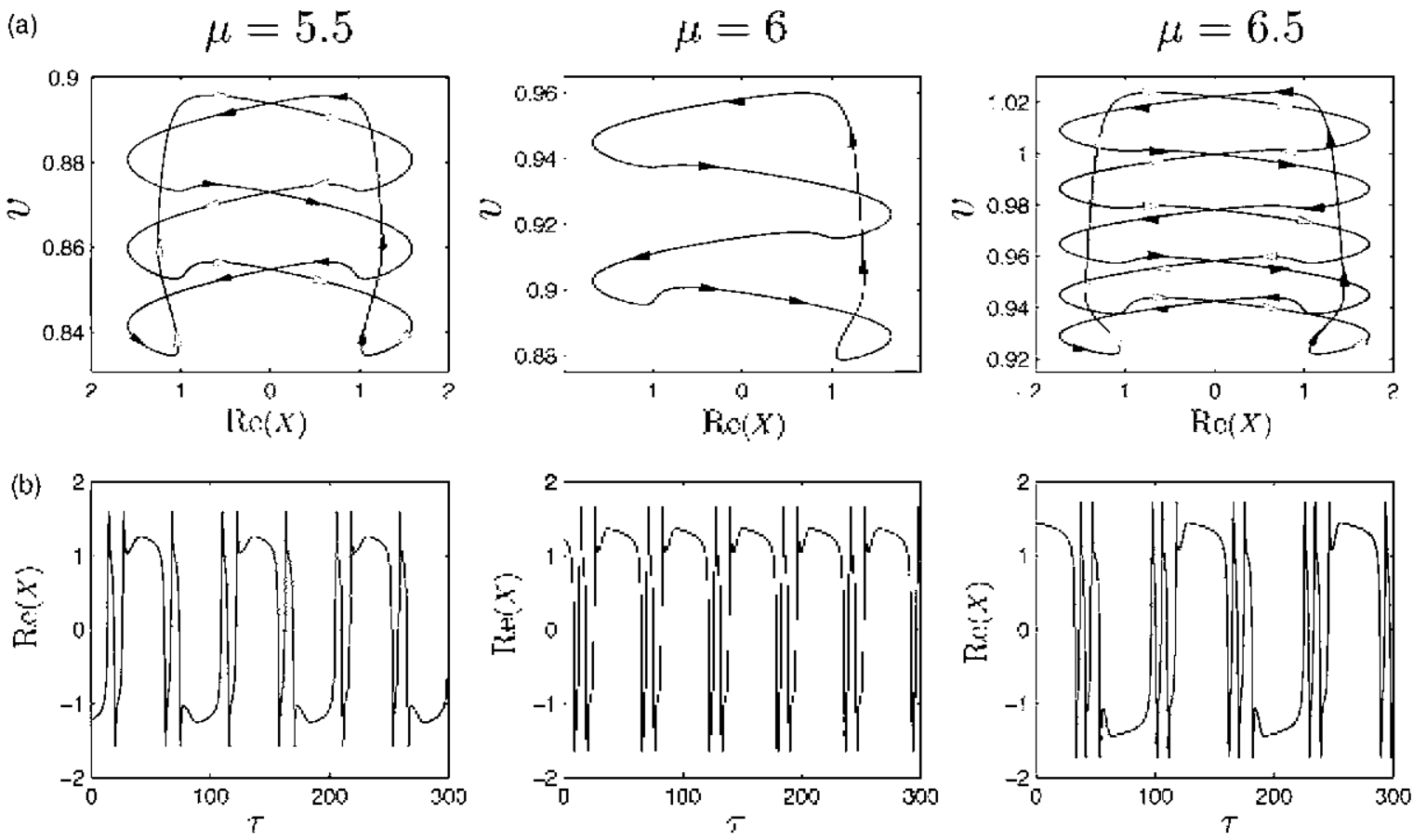

Fig. 13. Case III. (a) Projection onto the $(\operatorname{Re}(X), v)$ plane of stable symmetric and asymmetric periodic orbits in successive periodic windows in Fig. 12. (b) The corresponding time series $\operatorname{Re}(X(\tau))$. 

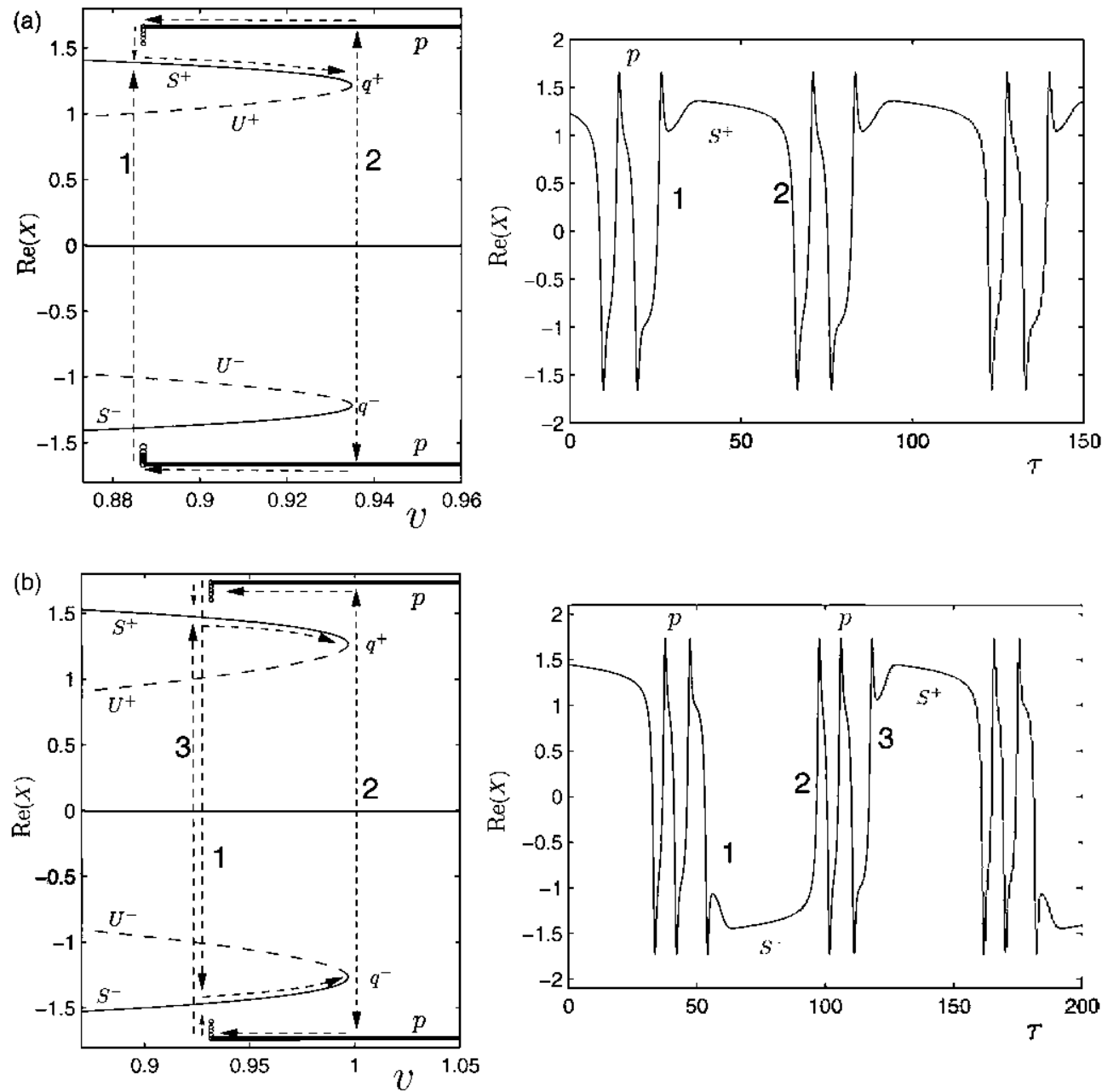

Fig. 14. Case III. The slow manifold for the system (42) lies within $O(\varepsilon)$ of the manifolds $S^{+}, U^{+}$of steady states, and the manifold $p$ of periodic orbits, of Eqs. (42) with $\varepsilon=0$. The slow drifts in $v$ that occur when $0<\varepsilon \ll 1$ are indicated by broken 'horizontal' arrows; the fast phase results in 'vertical' jumps. These are indicated by vertical aurows, and labeled by integers to indicate the corresponding transition in the associated time series $\operatorname{Re}(X(\tau))$. (a) $\Lambda$ symmetric relaxation oscillations of Eqs. (42) when $\mu=6.0$ and $\varepsilon=0.01$. (b) $R_{\mid} R_{2}$-symmetric relaxation oscillations of Eqs. (42) when $\mu=6.5$ and $\varepsilon=0.01$.

labeled with the number 2 . The system then drifts towards the left along the corresponding slow manifold $M_{p}$ until it reaches the vicinity of the $\varepsilon=0$ heteroclinic connection, where the oscillations disappear and so does the associated slow manifold $M_{p}(0<\varepsilon \ll 1)$. With the disappearance of $M_{p}$ the system is forced to either jump toward $S^{+}$or toward $S^{-}$. Which of these two outcomes takes place is determined by the phase of the trajectory near $U^{+}$or $U^{-}$. These states are saddles with one unstable direction and three stable directions; for example, the eigenvalues of $U^{ \pm}$ at $\mu=6.5$ are $0.6547,-0.9999 \pm 14.236 \mathrm{i}$ and -2.65405 . Thus, the least stable eigenvalue is in fact complex, and this is so in the other periodic windows as well. The time series show clearly that when the drift along $M_{p}$ ends the system approaches either $U^{+}$or $U^{-}$along its stable manifold; what happens thereafter depends on which part of its unstable manifold is followed. If the unstable manifold of $U^{+}$(say) takes the system to $S^{+}$(as in Fig. 14a) the fast phase (labeled 1) terminates on $S^{+}$and the system thereafter drifts towards the right along $M_{S}^{+}$. The resulting 

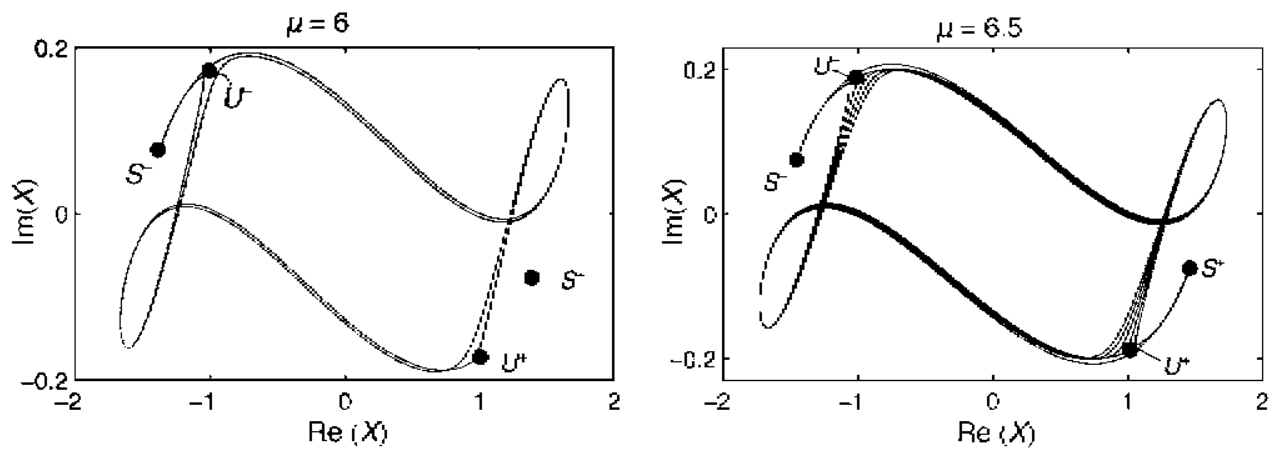

Fig. 15. Case III. Projections of (a) an asymmetric solution at $\mu=6.0$ and (b) an $R_{\mid} R_{2}$-symmetric solution at $\mu=6.5$ on the $(\operatorname{Re}(X)$, $\operatorname{Im}(X))$ plane, both calculated with $s=0.01$, showing the role played by the stable manifold of $U^{+}$in determining the part of the slow stable manifold followed by the solution. The actual trajectory spirats around this manifold.

oscillation is an asymmetric relaxation oscillation. In contrast, if the unstable manifold of $U^{+}$takes the system to $S^{-}$(cf. Fig. 15) the fast phase terminates on $S^{-}$and the system thereafter drifts towards the right along $M_{S}^{-}$. In Fig. 14b, this transition is labeled 1 ; the accompanying panel shows the corresponding signature in the time series. When the slow phase terminates the sy stem jumps back to the large amplitude periodic state (transition 2) and drifts along it to the left, but this time when it falls off $M_{p}$ in a transition labeled 3 it goes to $U_{-}$and by symmetry follows its unstable manifold towards $S^{+}$. The resulting trajectory is $R_{1} R_{2}$-symmetric. Fig. 15 shows the oscillations of Fig. 13 in a projection that highlights the role of the stable manifold of these unstable steady states as a separatrix between the two slow phases of the relaxation oscillation.

We now turn to the sequence of transitions revealed in Fig. 12. The bifurcation diagrams in Fig. 16 shows what happens in all transitions from an asymmetric oscillation to a symmetric one, and from a symmetric one to an asymmetric onc, as $\mu$ increases, while Fig. 17 shows the period of the corresponding solutions, also as a function of $\mu$ (boxed regions). Each transition is accompanied by a stability gap within which chaotic dy namics (symmetric and asymmetric) are found, and each new periodic solution emerges out of such a region in a saddle-node bifurcation. Fig. 17 also shows that in each transition the period of the stable oscillation jumps by an approximately constant amount, and subsequently decreases along both the stable and the unstable branches away from the saddle-node bifurcation.

Fig. 18 focuses on a branch of asymmetric relaxation oscillations that first appears in a saddle-node bifurcation ncar $\mu=7 .($, and shows that as one follows the stable branch from the saddle-node towards larger $\mu$ one encounters an interval of $\mu$ in which the period drops precipitously (Fig. 18a, inset). Just after this point the stable (asymmetric) relaxation oscillation loses stability via period-doubling (not shown). Fig. $18 \mathrm{~b}$ shows the time series at the point marked $3(\mu=7.3118)$ just before this loss of stability, and suggests that this loss of stability is a consequence of the fact that the trajectory is beginning to follow the unstable manifold before peeling off towards $S^{ \pm}$. By point $5(\mu=7.5533)$ the trajectory follows the unstable manifold $U^{-}$all the way to the saddle-node at $q^{-}$. For yet larger $\mu$ the oscillation remains unstable but the trajectory departs from $U^{-}$in the opposite direction (see point 6 , $\mu=10.0$ ). These results indicate that with increasing $\mu$ the trajectory comes closer and closer to the hyperbolic steady states $U^{ \pm}$resulting in longer and longer drift along the unstable manifold $M_{U}$. This fact also suggests an increasing role for the leading eigenvalues of $U^{ \pm}$. In the present case the least stable eigenvalue $\lambda_{\mathrm{s}}$ is complex, but the unstable cigenvalue $\lambda_{\mathrm{u}}<\left|\operatorname{Re}\left(\lambda_{\mathrm{s}}\right)\right|$. Thus no chaotic dynamics are expected. Note that in contrast to Case II in the present case the drift along $U^{ \pm}$is not triggered by a bifurcation in the fast system, and so is not the result of a canard. 

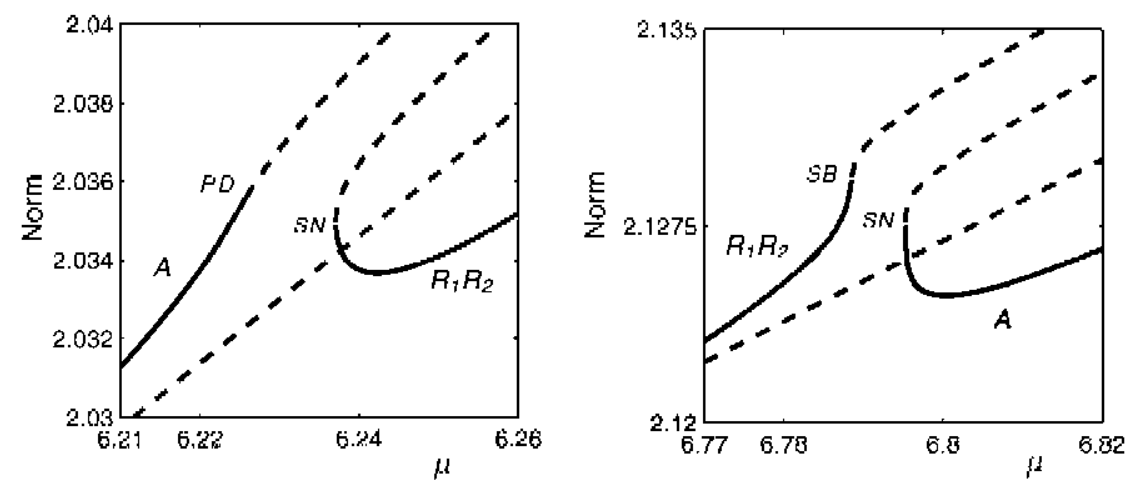

Fig. 16. Case III. J)tail of the hifurcation diagrams near the transition from asymmetric to symmetric relaxation oscillations near (a) $\mu=6.23$, and (b) $\mu=6.7 \%$. Solid (broken) lines indieate stable (unstable) solutions. The labels SN, SB and PI) denote saddle-node, symmetry-breaking and period-doubling bilurcations, respectively. The original (and unstable) oscillations correspond to the straight dashed line and undergo no bifurcations in this regime.

Stable bursting behavior of the type shown in Figs. 13,14 and 18 becomes increasingly dramatic as $\mu$ is increased, revealing ever more clearly the two slow phases and the rapid transition between them. In Fig. 19a, we show a stable oscillation for $\varepsilon=0.01$ and $\mu=9.0$ projected onto the $\left(\|(X, Y)\| \equiv \sqrt{\|X\|^{2}+\|Y\|^{2}}, v\right)$ planc, cf. Fig. 14, and superposed on the slow manifold computed with $\varepsilon=0$. We see that during one part of the slow

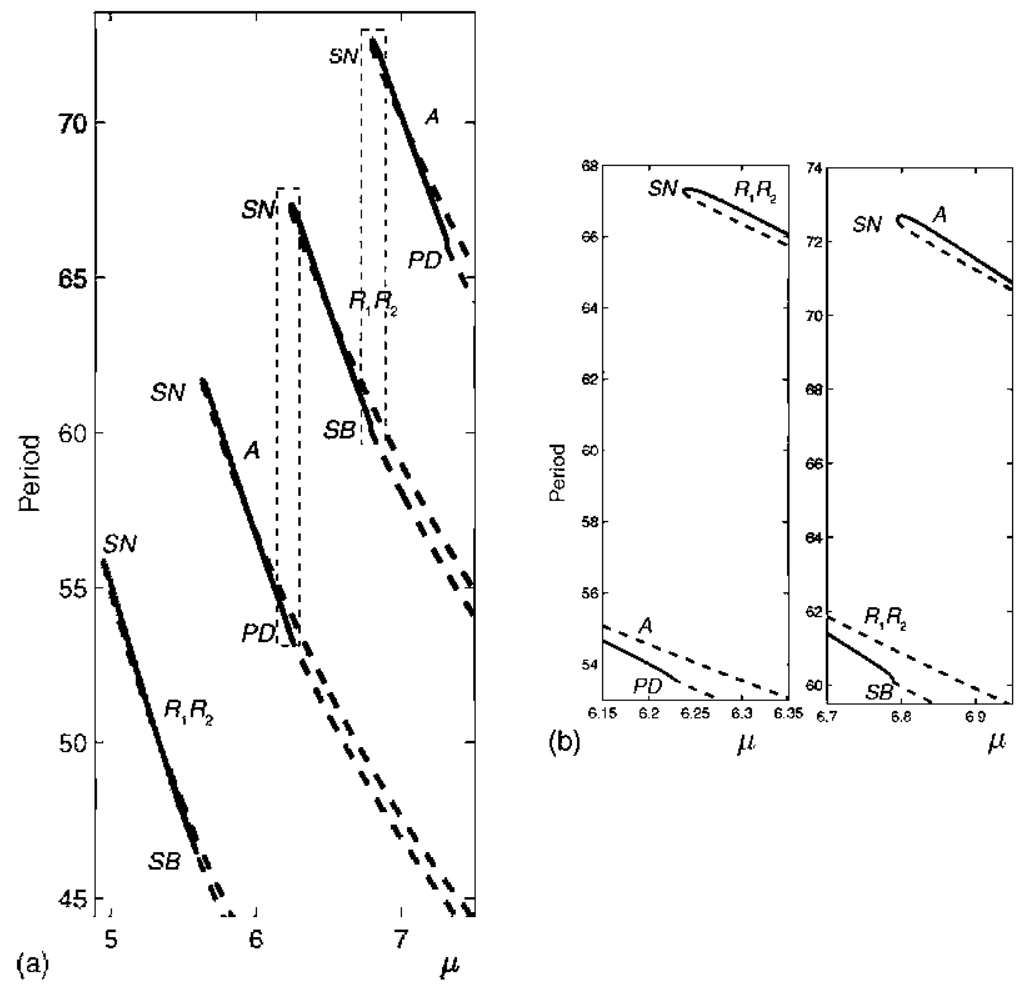

Fig. 17. Case III. AItemating sequence of $R_{1} R_{2}$-symmetric and asymmetric relaxation oscillations. The diagram shows the period (half-period) of asymmetric (symmetric) periodic relaxation oscillations as a function of $\mu$. Solid (dashed) line corresponds to stable (unstable) states. The labels SN, SB and PI) denote saddle-node, symmetry-breaking and period-doubling bifureations, respectively. 

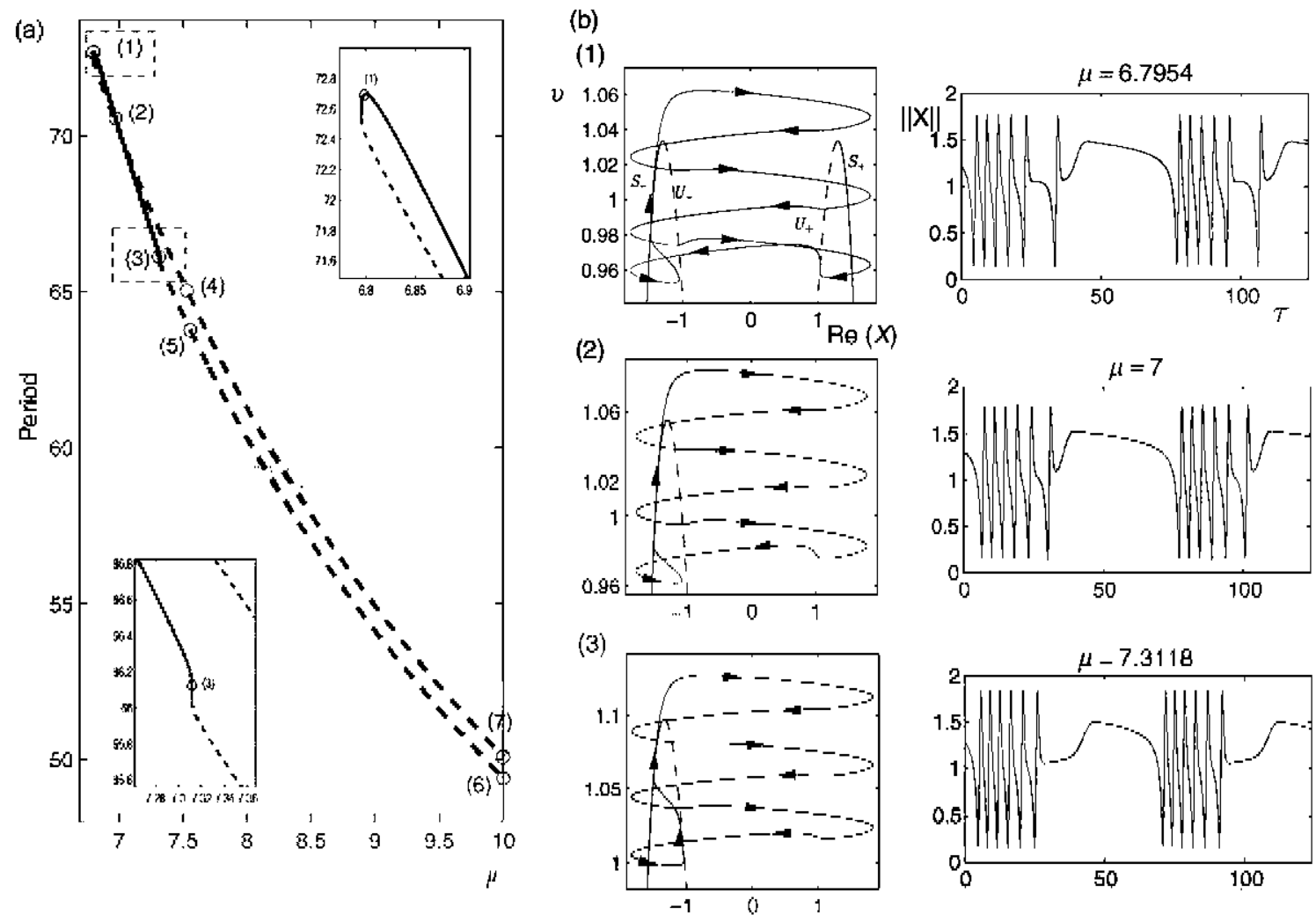

(c)

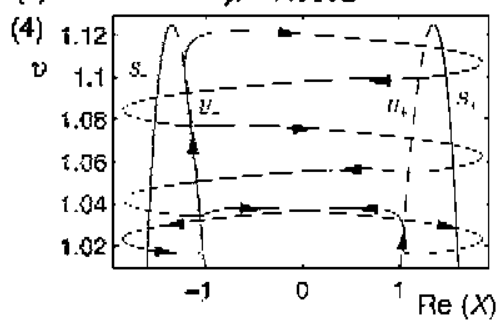

$\mu=10$

(6)

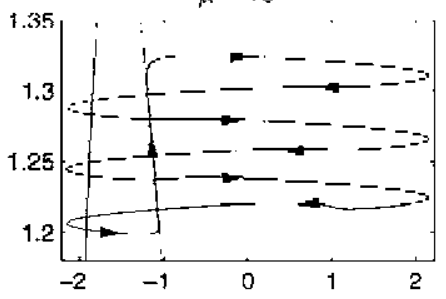

$\mu=7,5533$

(5)

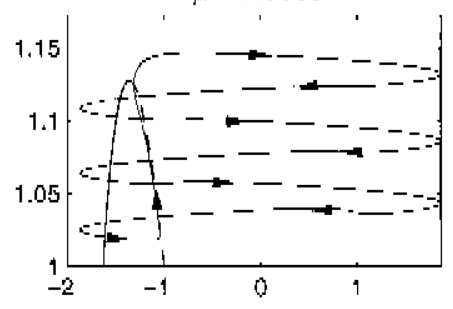

(7)

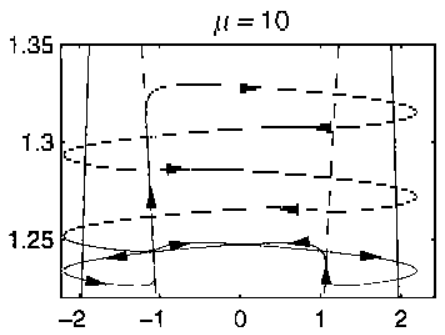

Fig. 18. Case III. (a) Period of asymmetric relaxation oscillations as a function of $\mu$. Solid (dashed) lines correspond to stable (unstable) states. (h) Stable relaxation oseillations corresponding to the open cireles (1-3) in figure (a) projected on the (Re $(X)$, v) plane together with the corresponding time series $\|X(\tau)\|$. (c) Instalsle relaxation oscillations corresponding to the remaining open cireles (4-7) in figure (a) in the same projection. 
phase (labeled 1) the system drifts towards smaller $v$ along the slow manifold $M_{p}$ of stable periodic solutions $p$. Near the hetcroclinic connection in the fast system the oscillations end and the system undergoes a rapid transition to the stable manifold $M_{S}$ indicated by a vertical arrow. Thereafter it enters a new slow phase, labeled 2 , and drifts in the direction of increasing $v$, towards the fold point $q$, where the slow phase ends and the system makes a rapid transition back to $M_{p}$. This transition is also indicated by a vertical arrow. The small cusp-like fealure at the onset of phase 2 results from a brief visit of the trajectory near the unstable slow manifold $M_{U}$, before being ejected towards the stable slow manifold $M_{S}$. As $\mu$ increases and the next symmetry-switching transition is approached the solutions spend more and more time near $M_{U}$, and hence involve longer and longer episodes of drift along $M_{U}$. Fig. $19 \mathrm{~b}$ and c show two examples of this behavior, differing only slightly in the value of $\mu$. In the first of these the system drops to the unstable steady state at the end of the slow phase 1 , and then drifts along $M_{U}$ towards larger $v$ before a sudden jump to $M_{S}$. The slow drift continues along $M_{S}$ towards the fold on $\Sigma$ where the system jumps to the oscillatory state $p$, and the slow phase 1 recommences. In contrast, the solution in Fig. 19c jumps from $M_{U}$ to $M_{p}$ (instead of $M_{S}$ ) and so begins to drift towards smaller $v$ but the next time, instcad of following $M_{U}$, it jumps to $M_{S}$ and begins to follow the standard relaxation oscillation scenario. The remarkable sensitivity to the value of $\mu$ is evidently a consequence of the amplification of small differences in the approach of the trajectory towards $M_{U}$ by the unstable eigenvalue of $U$, and heralds the transition from asymmetric oscillations to symmetric ones: in effect, with each half-turn around $U^{ \pm}$the trajectory changes the direction in which it leaves $U^{ \pm}$, and hence its symmetry. In addition its period jumps by a finite amount, a phenomenon we have already noted. When the number of turns increases monotonically with $\mu$ there will be an infinite sequence of symmetry-changing transitions, and hence a sequence of periodic windows with alternating symmetric and asymmetric relaxation oscillations. Thus each saddle-node bifurcation creates a pair of periodic orbits with an extra half-turn. Moreover, since it takes a finite interval in $\mu$ to change the frequency sufficiently to add a halfturn these symmetry-changing transitions cannot accumulate, in contrast to the cascades of symmetry-switching gluing bifurcations that occur in other $D_{2}$-symmetric systems [21]. As documented above this is the case for the parameters of Case III; for other choices such as Case II with $0.35 \leq \gamma<0.4$ (not shown) the number of transitions is finite.

\subsection{Case IV: Delayed loss of stability and chaos}

Case IV is quite complex because of the proximity to the saddle-node bifurcation on the $P_{+}$branch of two other bifurcations, the symmetry-breaking Hopf and steady state bifurcations $H$ and SB, respectively. The former interaction, i.c., saddle-node/Hopl, is responsible for the properties of the oscillations created at the Hop f bifurcation. This codimension-two bifurcation is much studied $[19,46]$ and its unfolding corresponding to Fig. 2d confirms not only the presence of a branch of oscillatory solutions that bifurcates towards larger $\mu$ but also shows that this branch must be initially unstable before acquiring stability in a tertiary torus bifurcation. This theoretical prediction is confirmed in Fig. $20 \mathrm{a}$ where the torus bifurcation is labeled TR $(\mu \approx 1.00136)$. The figure shows, however, that the interval in which the periodic oscillations (thick line) are stable is quite narrow, and that these oscillations lose stability at larger $\mu$ at a second tonus bifurcation $(\mu=1.0083)$. We presume that this loss of stability is a consequence of the proximity of these parameter values to the second codimension-iwo interaction: the saddle-node/pitchfork bifurcation. Indeed, Fig. 20a shows that the periodic oscillations eventually become stable again (at a third torus bifurcation) before transferring stability, via a Hopf bifurcation, to the mixed modes $M$ created at SB. This Hopf bifurcation is in turn found in the appropriate unfolding of the saddle-node/pitchfork interaction.

It is important to emphasize that the oscillations created in the Hopf bifurcations on the $P_{+}$and $M$ branches have distinct symmetry properties. The former are $R_{1}$-symmetric and disappear in a gluing bifurcation at $\mu=\mu_{\mathrm{g}} \approx 1.03$ involving the larger amplitude $P_{+}$state. The latter produces a pair of asymmetric oscillations, which glue together as $\mu$ approaches $\mu_{\mathrm{g}}$ from above (see Fig. 20a). There are therefore two possible sources of complex behavior in this parameter regime, the first associated with the global bifurcation at $\mu=\mu_{\mathrm{g}}$, and the second associated with 

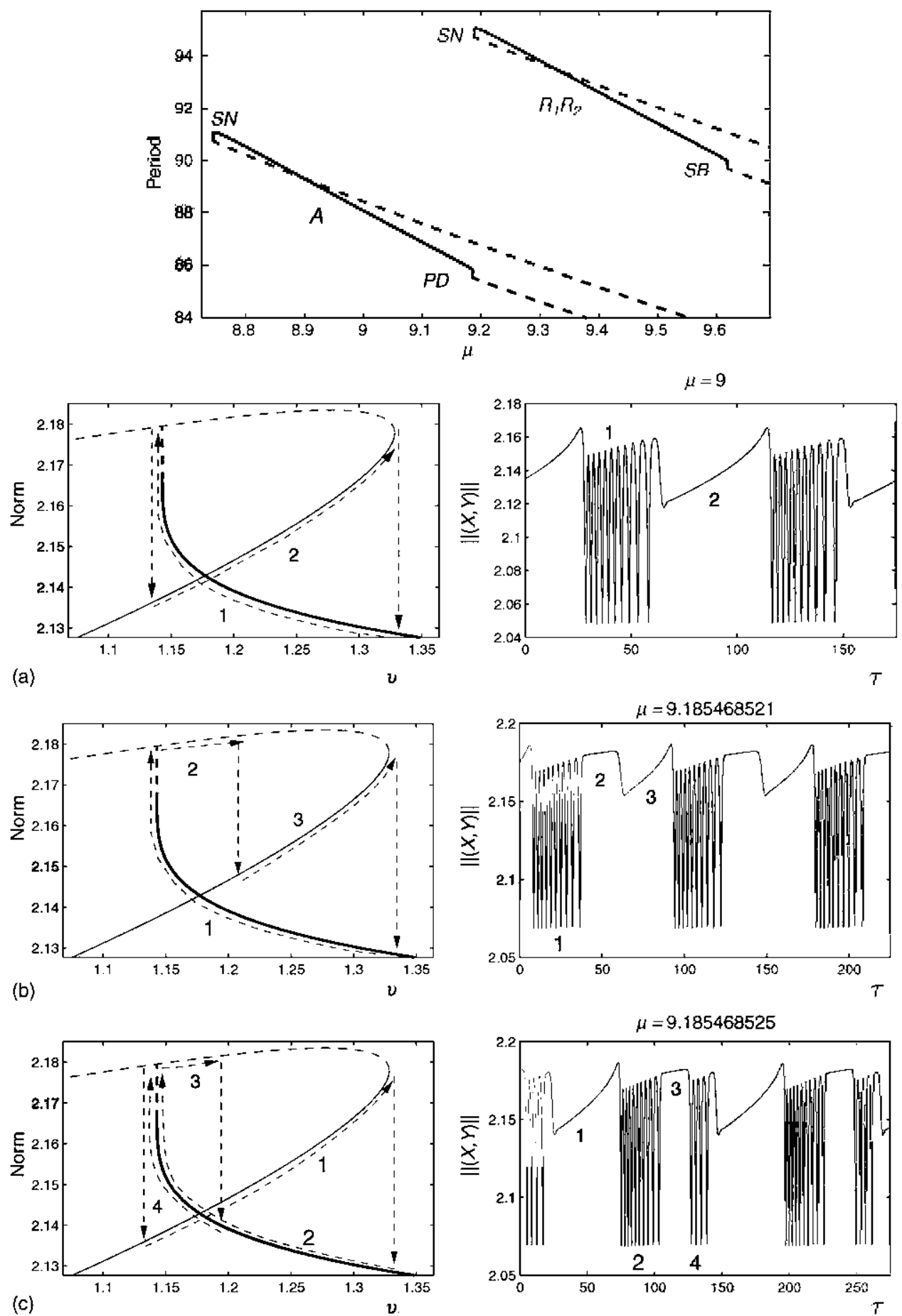

Fig. 19. Case III. Stable asymmetric relaxation oscillations for (a) $\mu=9$, (b) $\mu=9.185468521$ and (c) $\mu=9.185468525$. The top panel shows the location of these solutions in a period vs. $\mu$ plot (Fig. 17). The slow drifts, present when $0<\varepsilon \ll 1$, are labeled with numbers to indicate the corresponding phase in the accompanying time series $\|(X, Y)\|$, compuled from Eqs. (42) with $s=0.01$. The arrows indicate the direction of drift as well as the fast transitions. 

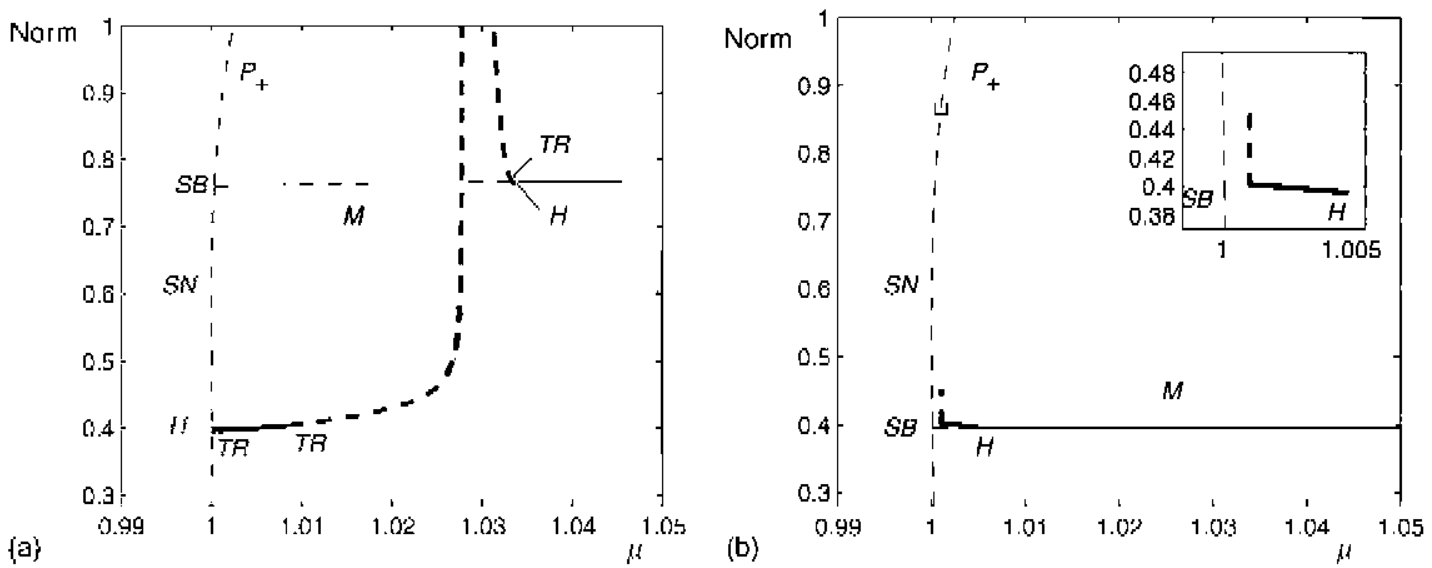

Fig. 20. Case IV, corresponding to Fig. $2 \mathrm{~d}$ for (a) $\gamma=-0.35$ (mean flow included) and (b) $\gamma=0$ (mean flow absent). Thin solid (dashed) lines correspond to stable (unstable) steady states. Thick solid (dashed) lines correspond to branches of stable (unstable) periodic orbits generated in Hopf bifurcations on $P_{1}$ and $M$, indicated by the letter $H$. Saddle-node, symmetry-breaking pitchfork and torus bifurcations are indicated by SN, SB and TR, respectively. In (b) the symbol $\sqcap$ indicates the homoelinie global bifureation where the branch of periodics orlsits, created at $H$, terminates. Note the rapid growth in the oscillation amplitude (inset) as this point is approached, ef. [35].

the termination of the torus branches. The former is not directly responsible for the observed chaotic dynamics (see below) since the eigenvalues of $P_{+}$at $\mu_{\mathrm{gg}}$ are real, and the leading unstable eigenvalue is smaller than the (magnitude of the) leading stable one. Analysis of the saddle-node/Hopf intcraction shows that the associated torus branch terminates in general in a global bifurcation as well. As shown by Guckenheimer [17] and in more detail by Kirk $[24,25]$ this bifurcation is generically associated with thin wedges of chaotic behavior. We expect this type of behavior at the end of the first torus branch, and similar behavior may be associated with the torus branches created when the various periodic oscillations identified below lose stability as well. Fig. 20b shows that when the coupling to the mean flow is omitted the behavior of the system simplifies dramatically. However, despite the differences in behavior summarized in Fig. 20a and b there is clear correspondence between the two figures. This correspondence is a consequence of both the small value of $\varepsilon$ which is responsible for replacing the steady states $M$ in Fig. 20b by slowly drifting states that form the slow phase of the oscillations created in the Hopf bifurcation on $P_{+}$(Fig. 20a), and of the small value of the mean flow $v$ associated with these oscillations. This correspondence is explored in greater depth below.

In Fig. 21 , we show a two-frequency attractor obtained at $\mu=1.0005$, that is, immediately after the trivial state becomes unstable ( $\mu_{0}=1.00045$ ), with the mean flow included (Fig. 20a). Fig. 22a shows the corresponding time serics. This attractor appears to describe oscillations about the small amplitude $R_{1}$-symmetric oscillation created at the Hopf bifurcation on the $P_{+}$branch. The absence of exact $R_{1}$ symmetry suggests that this attractor is related to the symmetry-breaking torus bifurcation at $\mu \approx 1 .(0) 136$. This bifurcation appears to be supercritical, producing stable two-frequency oscillations in $\mu \lesssim 1.00136$. These are initially almost $R_{1}$-symmetric but grow increasingly asymmetric as $\mu$ decreases. The $R_{1}$-symmetric oscillations undergo a second torus bifurcation at $\mu \approx 1.0083$ but this time the bifurcation is subcritical, i.e., the resulting two-frequency oscillations are present in $\mu<1.0083$ but are now unstable. However, Fig. 23 shows that this observation only scratches the surface of the complexity that is present in this parameter regime. In addition to the $R_{1}$-symmetric oscillations just mentioned (Fig. 20 and lowest curve in Fig. 23) there is a large number of additional branches of $R_{1}$-symmetric oscillations, four of which are included in the figure. These are labeled (a-d) and are stable near minimum period (solid lincs) and unstable elsewhere (dashed lines). Fig. 24 shows the projections of these stable oscillations in four cases, one from each branch. It is clear that the oscillations corresponding to the different branches differ in the number of revolutions 
(a) $\mu=1.0005$

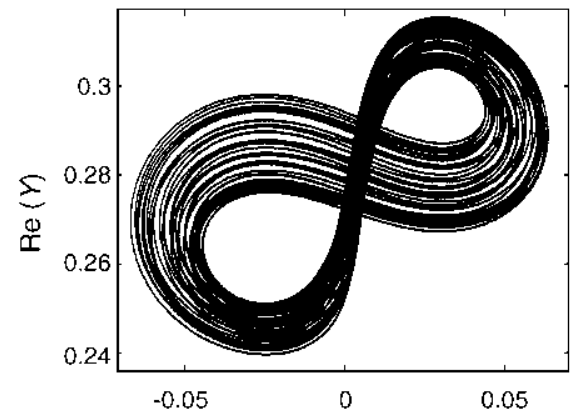

(b) $\mu=1.0175$

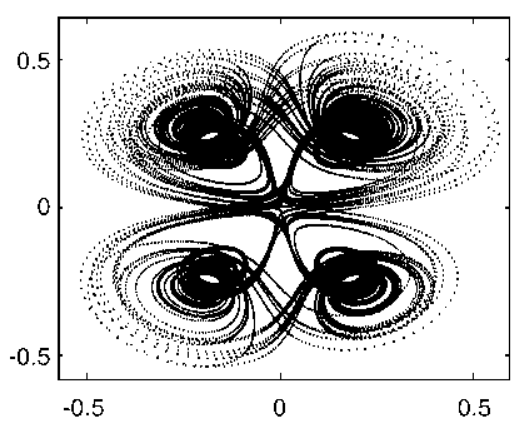

(c) $\mu=1.0205$

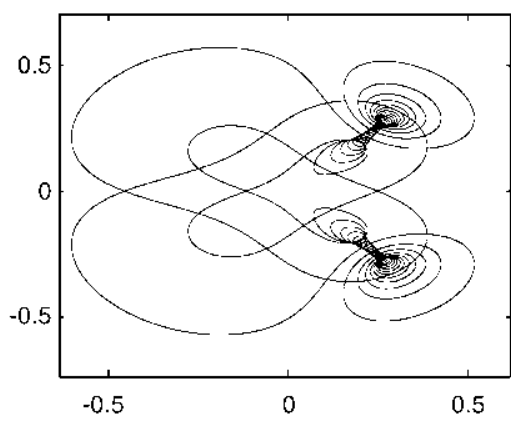

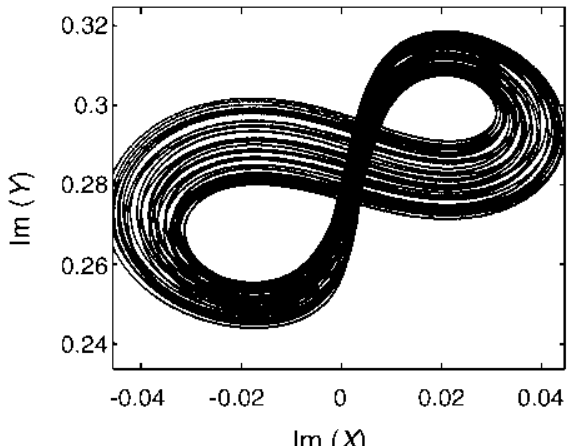
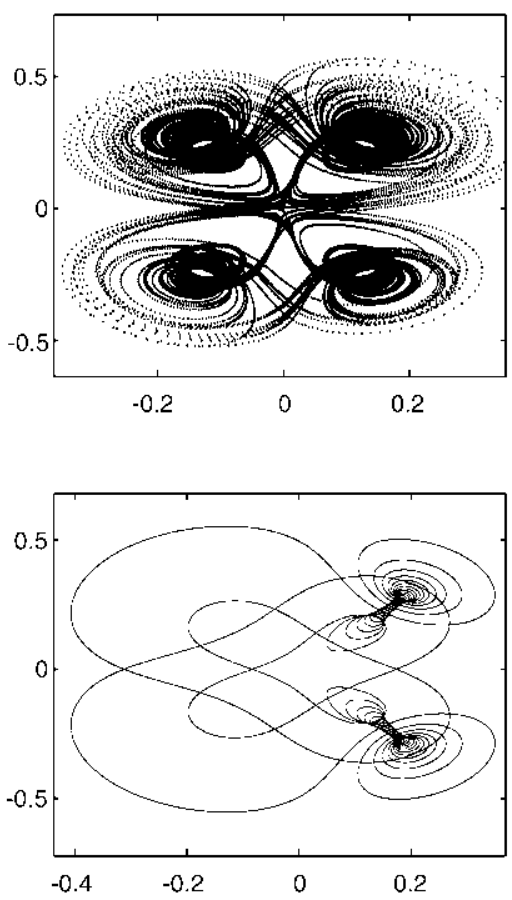

Fig. 21. Case IV. Stable attractors ol'Eqs. (26)-(28) for the parameters in Fig. 2d. (a) A torus at $\ell=1.0005$. (b) A chaotic attractor at $\ell=1.0175$. (c) $\wedge R_{2}$-symmetric periodic orbit at $\mu=1.0205$.

about the two mixed modes $M^{ \pm}$, and that multiple stable oscillations are present simultaneously. However, in all cases the oscillation period is of order $1 / \varepsilon$ as expected of relaxation oscillations. The torus bifurcations at which these oscillations acquire stability with increasing $\mu$ may be responsible for the presence of chaotic oscillations in this regime, such as that shown in Figs. $21 \mathrm{~b}$ and $22 \mathrm{~b}$, but the details of this process are beyond the scope of the present paper.

The behavior reported in Fig. 24 is also a consequence of the two distinct slow time scales present in the problem, i.e., of the small value of the parameter $\varepsilon$. The set $\Sigma$ of equilibria of Eqs. (42) with $\varepsilon=0$, given by $\boldsymbol{F}_{1}(\boldsymbol{X}, \boldsymbol{Y} ; v, \mu)=\boldsymbol{F}_{2}(\boldsymbol{X}, \boldsymbol{Y} ; v, \mu)=0$, consists of symmetry-related stable $\left(S^{ \pm}\right)$and unstable $\left(U^{ \pm}\right)$steady states. Of these the $S^{ \pm}$are created in pitchfork bifurcations from the trivial state as $|v|$ decreases. The $S^{ \pm}$states lose 
(a) $\mu=1.0005$

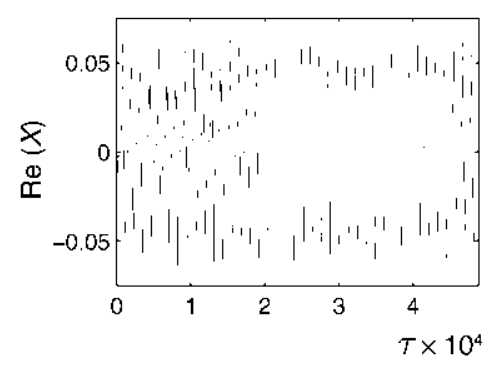

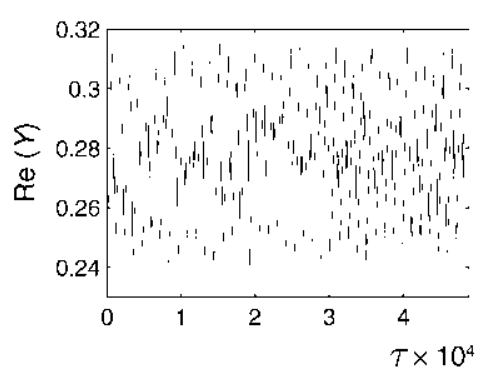

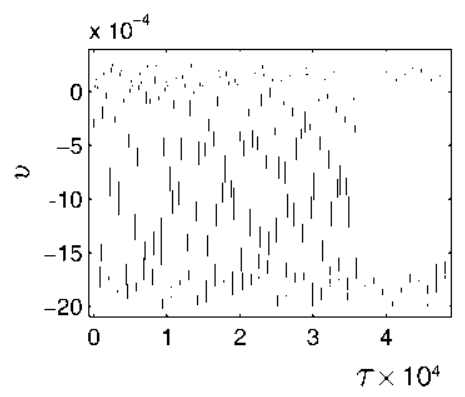

(b) $\mu=1.0175$
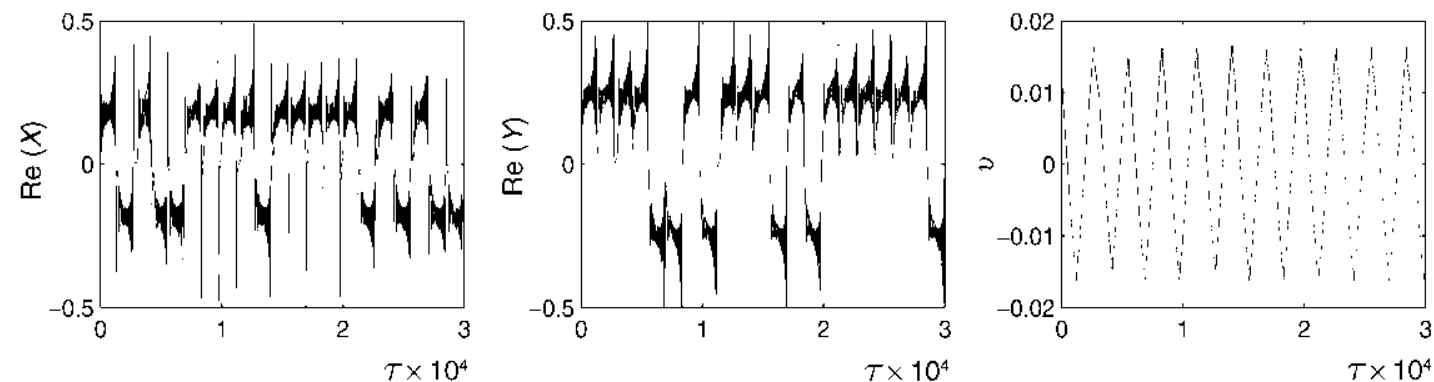

(c) $\mu=1.0205$
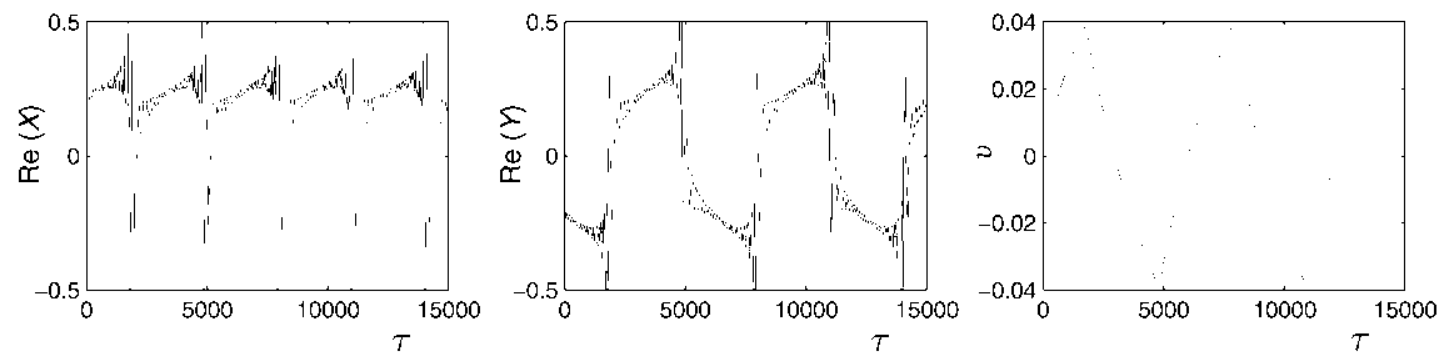

Fig. 22. Case IV. Time series corresponding to the attractors in Fig. 21.

stability via subcritical Hopf bifurcations at $h^{ \pm}$and turn into $U^{ \pm}$(see Fig. 25). The branches of periodic oscillations $p$ created in these Hopf bifurcations are always unstable and terminate in a homoclinic connection to an $R_{1}$ symmetric fixed point $U_{0}$. When $\mu=1.01185 U_{0}=(0,0,1.025,0.87303)$ and its eigenvalues are real: 0.458 , $-0.054,-1.945$ and -2.458 . The eigenvectors of the two leading eigenvalues are $V_{\mathrm{u}}=(0.8454,0.5341,0,0)$ and $V_{\mathrm{S}}=(0.7458,0.4389,0,0)$. The global bifurcation at $U_{0}$ is therefore a standard 'figure eight' gluing bifurcation with no associated chaos; its presence does not appear to be relevant for the dynamics shown in Fig. 24. Rather, the observed behavior appears to be a consequence of the fact that all four eigenvalues of $S^{ \pm}$are complex. When $0<\varepsilon \ll 1$ the solutions of Eqs. (42) drift slowly along the attracting slow manifolds $M_{S}^{ \pm}$associated with $S^{ \pm}$; this drift is in the direction of increasing $v$ for $M_{S}^{-}$and decreasing $v$ for $M_{S}^{+}$. As in Case III, when $\varepsilon$ is sufficiently small the fast motion undergoes a delayed loss of stability (cf. [2]), although this time no global bifurcation is involved. This behavior can be seen clearly in Fig. 26, computed for $\varepsilon=0.0001$ : the trajectory moves slowly along $M_{S}^{-}$, passes through a Hopf bifurcation (in the fast system) at $h^{-}$, and continues along the unstable manifold $M_{U}^{-}$for a 


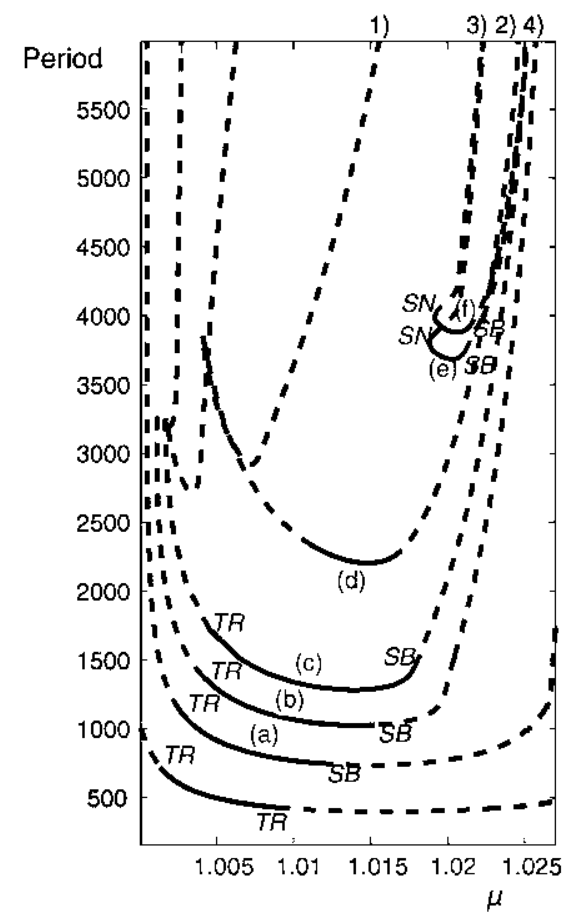

Fig. 23. Case IV. The period of several branches of periodic oscillations as a function of $\mu$ when $\varepsilon=0.001$. The branches (a d) are $R_{1}$-symmetric, while (e and f) are $R_{2}$-symmetric. Solid (dashed) lines indicate stable (unstable) solutions. Saddle-node, symmetry-breaking pitchfork and torus bifurcations are indicated by $\mathrm{SN}, \mathrm{SB}$ and TR, respectively.

time of order $1 / \varepsilon$. The trajectory then spirals away from $M_{U}^{-}$and towards $M_{S}^{+}$. Once it is sufficiently close to $M_{S}^{+}$ the same process repeats, but this time with $v$ decreasing. As shown in Fig. 27 the drift along the slow manifolds $M_{S}^{ \pm}$and $M_{U}^{ \pm}$is faster at larger values of $\varepsilon$. With less time to approach $M_{S}^{ \pm}$the oscillations appear less and less "damped" and the orbits begin to resemble those in Fig. 24.

The above point of view also permits us to understand the origin of the prominent cusp-like feature that develops on the higher branches of oscillatory states as $\mu$ decreases (Fig. 23). This structure is not due to an incipient global bifurcation. Instead what appears to be happening is the following. As $\mu$ decreases the real part $\lambda$ of the unstable eigenvalue at the detachment point also decreases, implying a longer oscillation period. At the same time the Hopf frequency $\omega$ at $h^{-}$also decreases. For the transition from $U^{-}$to $\mathrm{S}^{+}$one must make at least half a turn around $U^{-}$before detachment, otherwise the trajectory ends up on the large amplitude $U^{-}$state (see Fig. 25 ). This change in the type of oscillation becomes incvitable once $\omega / \pi$ falls below $\lambda$. Numerically we find that this condition is quite accurately satisfied at the tip of the cusp, while before the cusp is reached (i.e., for larger values of $\mu$ ) $\lambda \lesssim \omega / \pi$. After the cusp the unstable two-dimensional manifold of $U^{-}$starts to veer away from the stable manifold of $S^{+}$and begins to approach the three-dimensional stable manifold of the large amplitude $U^{-}$ state. As this happens the period drops slightly but the oscillation amplitude starts to increase. Fig. 28 shows the oscillations on either side of the (secondary) minimum in the period along branch (d) in Fig. 23. Thereafter the period increases rapidly as the trajectory spends more and more time drifting along the slow manifold associated with the large amplitude unstable state $U^{-}$instead of the stable small amplitude state $S^{+}$, thereby acquiring an altogether different appearance. On the left each branch eventually terminates in a homoclinic connection to the pure mode $P_{+}$contained in the slow manifold of $U^{-}$(see Fig. 29, panel 1), while on the right it terminates 

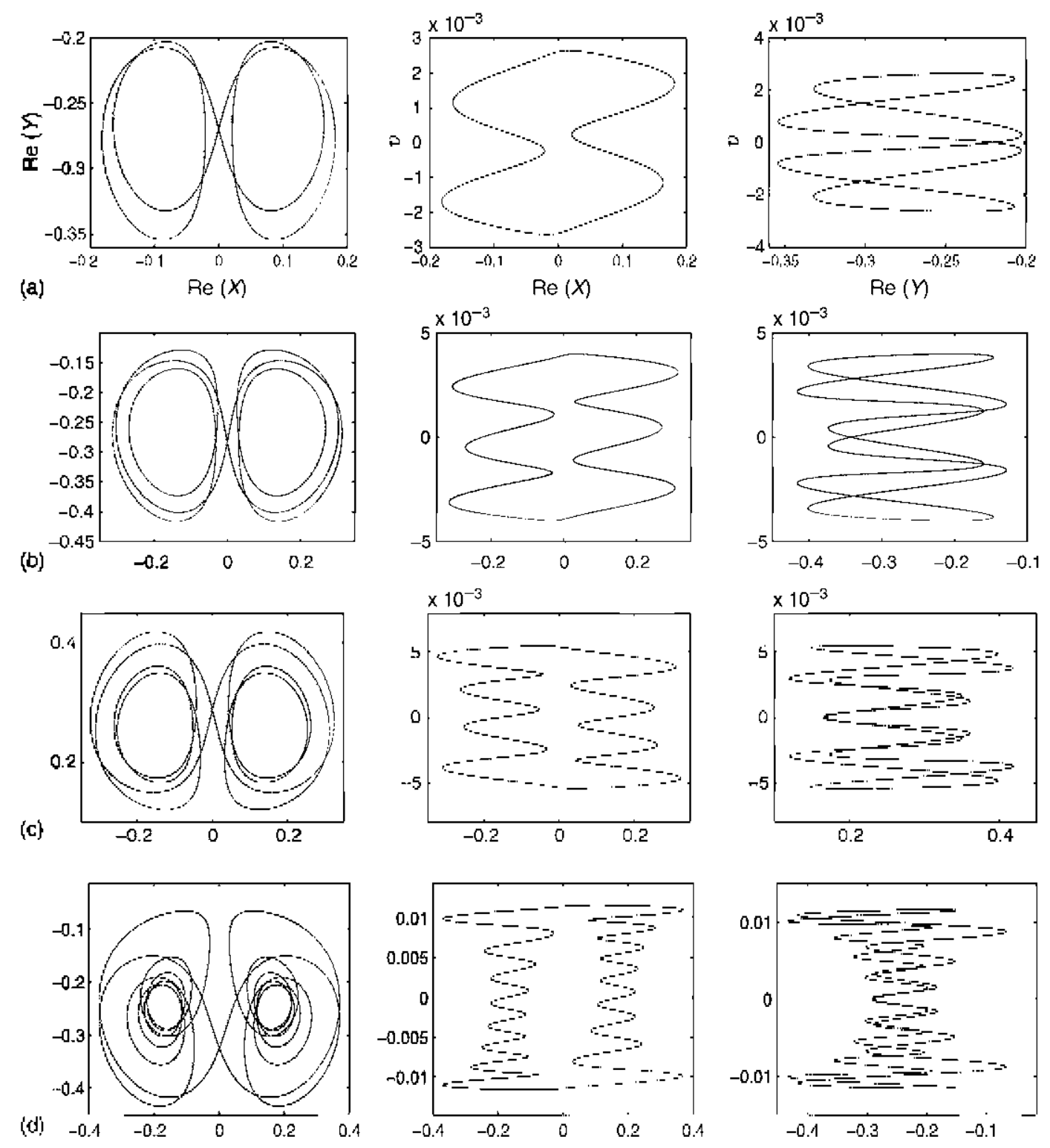

Fig. 24. Case IV. Fxamples of stable $R_{1}$-symmetric oscillations on the branches (a-d) in Fig. 23 at $\mu=1.01185$.

in a homoclinic connection to the origin (see Fig. 29, panel 2). Except for the complications arising from the presence of reflection symmetry much of the above phenomenology resembles that studied recently by Krauskopf and Wieczorek [26] and attributed to a nearby saddle-node/Hopf bifurcation with no stable fixed points [47], cf. Fig. 20a.

Fig. 23 also shows two branches of $R_{2}$-symmetric oscillations labeled (c and $)$ ). These are also stable near minimum period; a stable $R_{2}$-symmetric oscillation on branch (e) is illustrated in Figs. 21c and 22c. This type of solution can be described in an analogous manner to the $R_{1}$-symmetric orbits on branches (a-d) discussed above. 

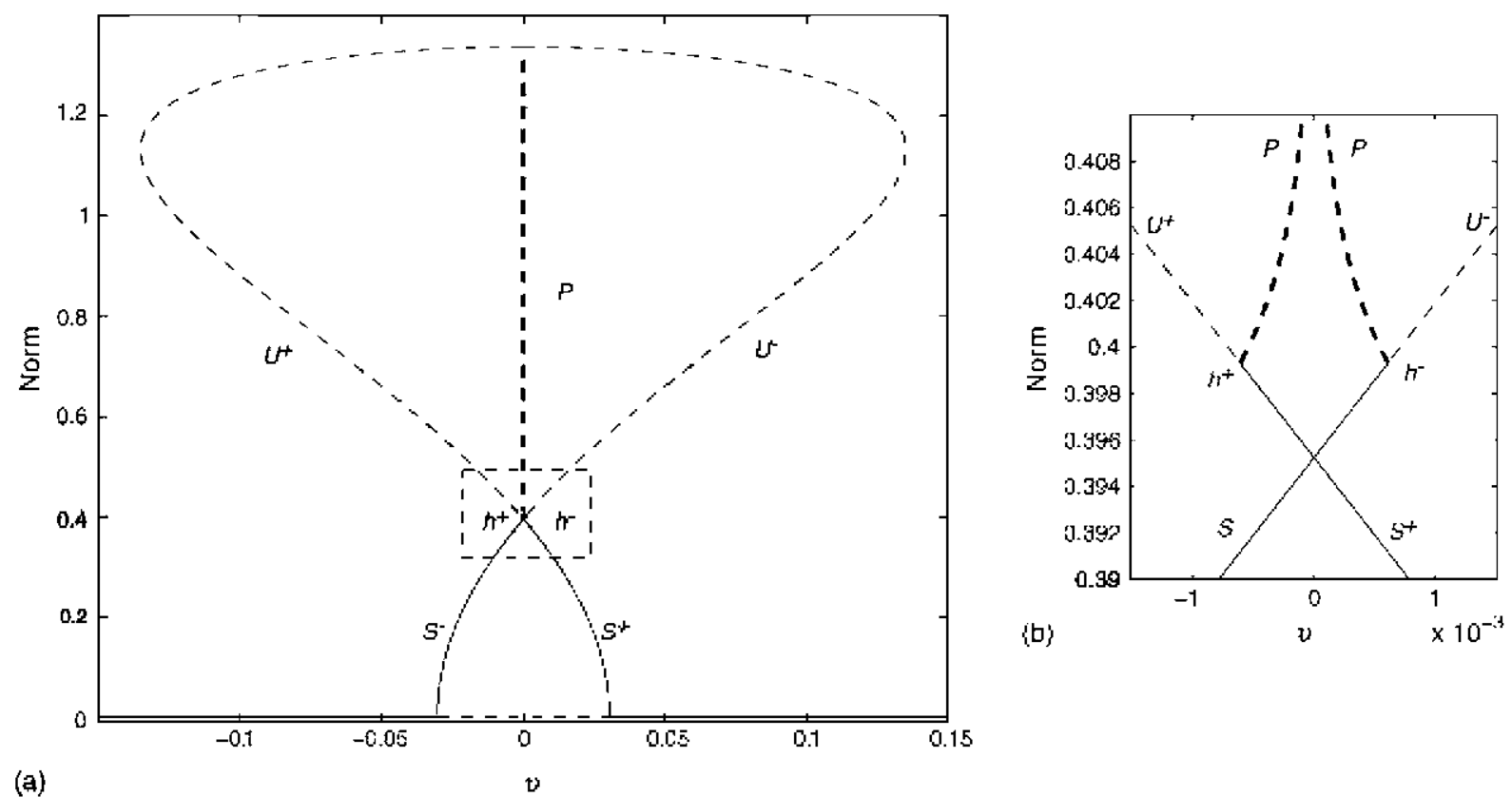

Fig. 25. Case IV. (a) Bilurcation diagram for Ecjs. (42) showing the norm $\|(X, Y)\|$ as a function of $v$ when $\mu=1.01185$ and $\varepsilon=0$. Thick dashed lines indicate branches of periodic orbits generated in Hopf bifurcations at $h^{ \pm}$. Thin solid (dashed) lines indicate stable (unstable) steady states. The remaining parameters are as in Fig. 21. (b) Detail of the bifurcation diagram in (a).

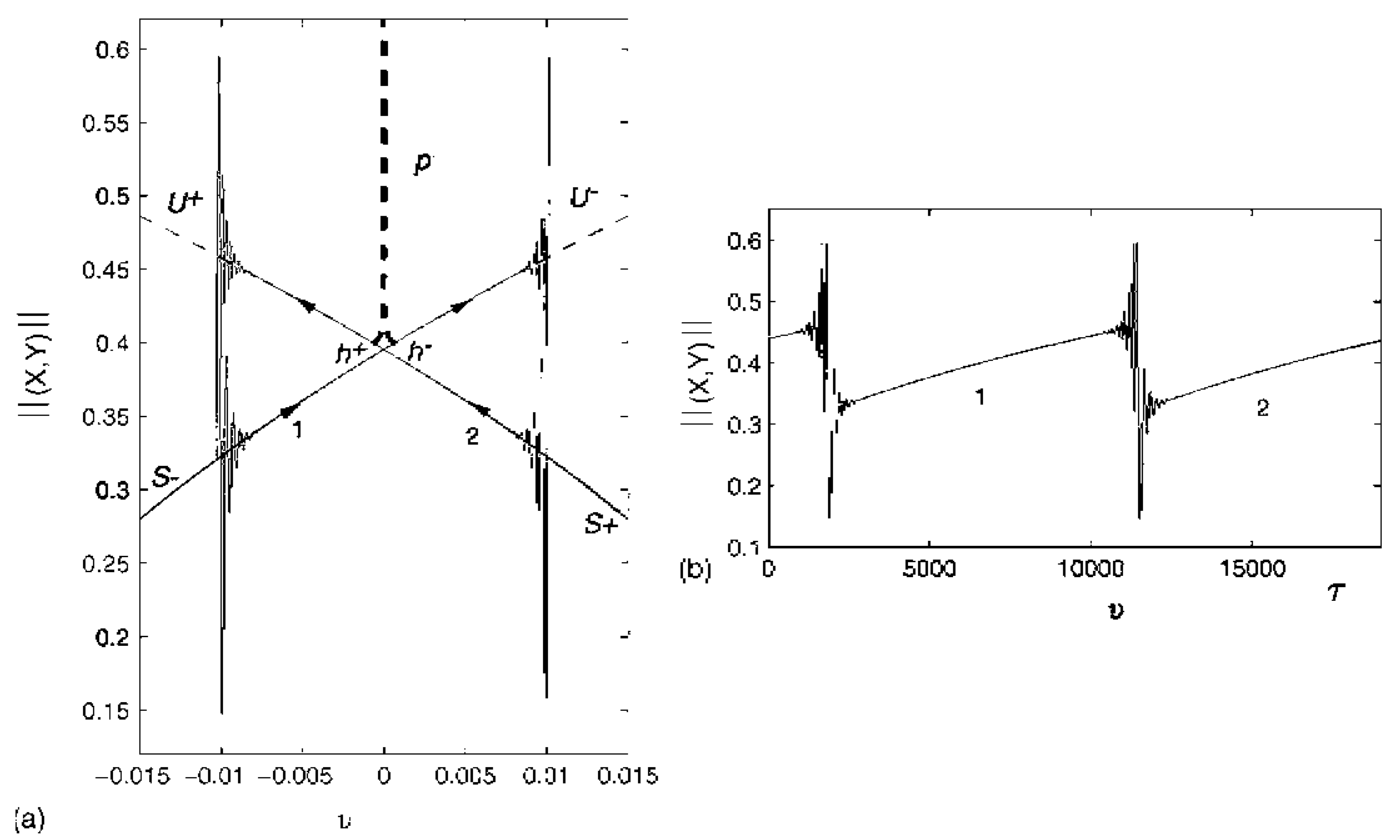

Fig. 26. Case IV. (a) Stable relaxation oscillation computed from Figs. (42) when $\mu=1.01185, \varepsilon=0.0001$, projected onto the (v, $\|(X, Y)\|)$ plane. (b) The corresponding time series $\|X(\tau), Y(\tau)\|$. The remaining paramelers are as in Fig. 21. 
F thol
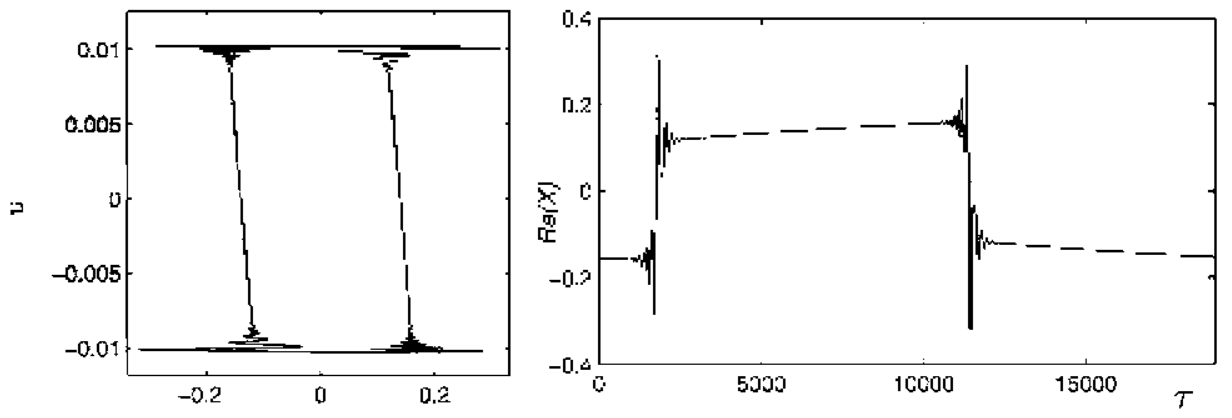

(a)

$\operatorname{Re}(x)$

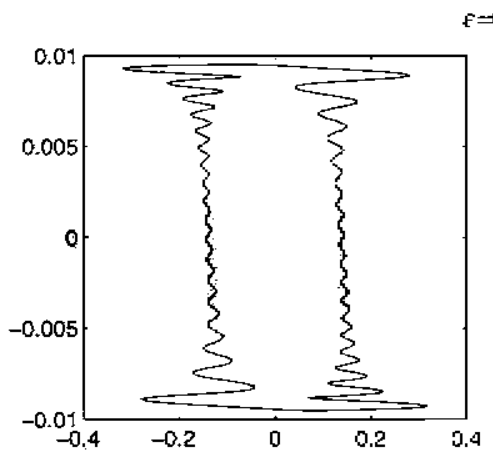

$f=0.00035$

(b)

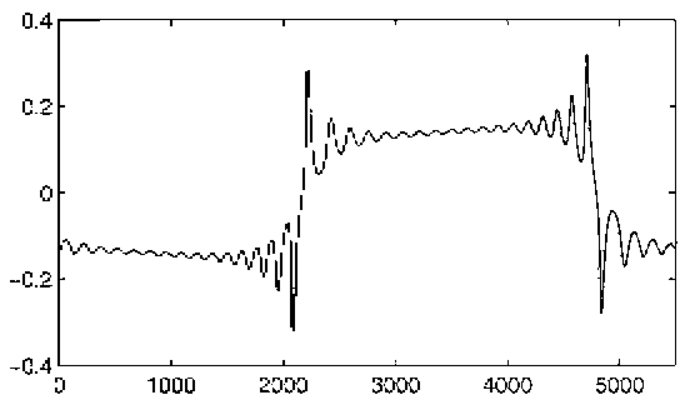

$\varepsilon=0.0005$
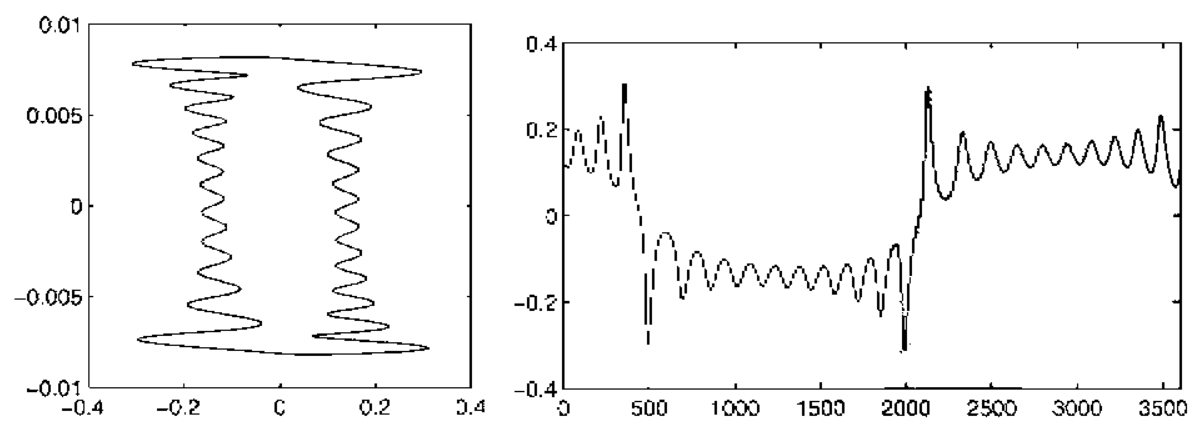

(c)

$\varepsilon=0.010]$
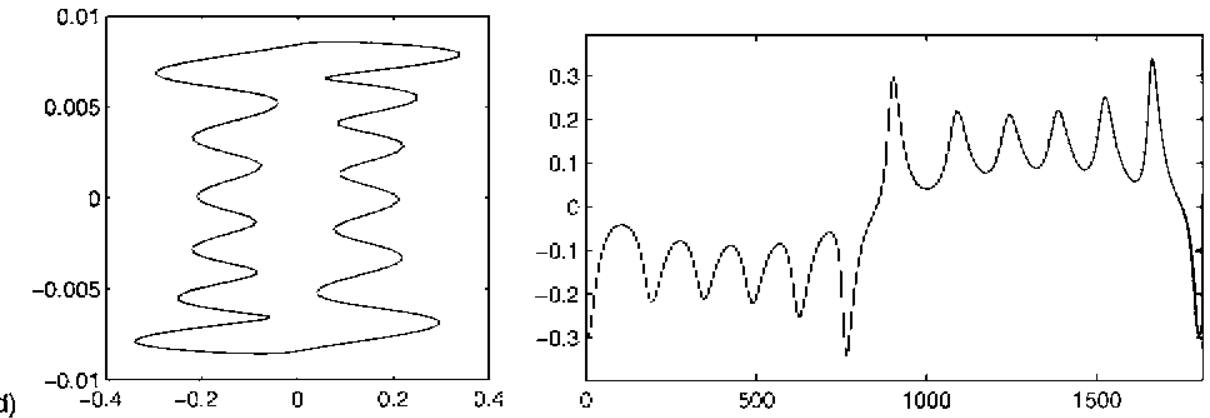

Fig. 27. Case IV. Stable $R_{1}$-symmetric oscillations for $\mu=1.01185$ and several different values of $\varepsilon$. The remaining parameters are as in Fig. 21. In each case the time series (riglit column) show at single period only. Note that (d) corresponds to a branch omitted from Fig. 23. 

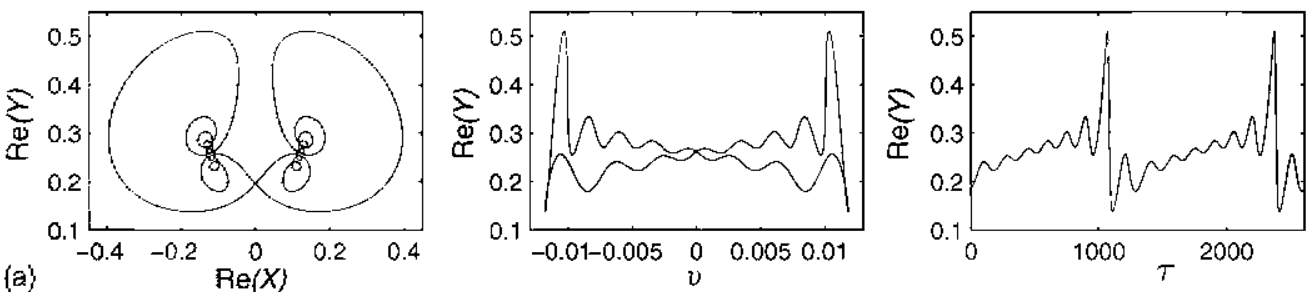

(a)
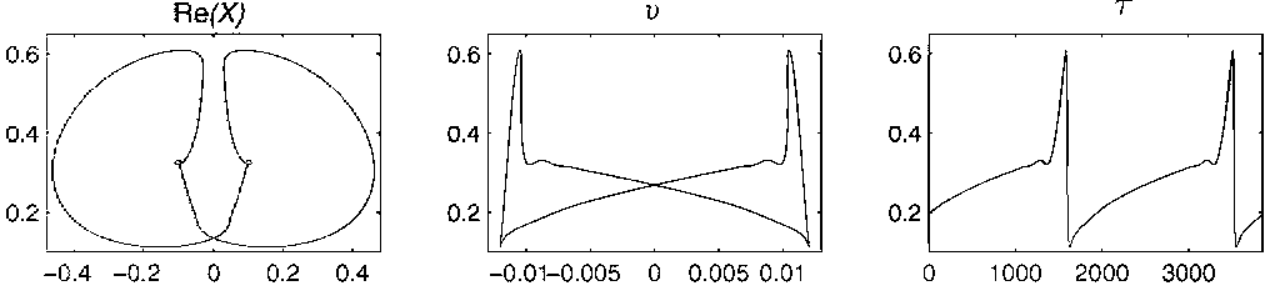

(c)
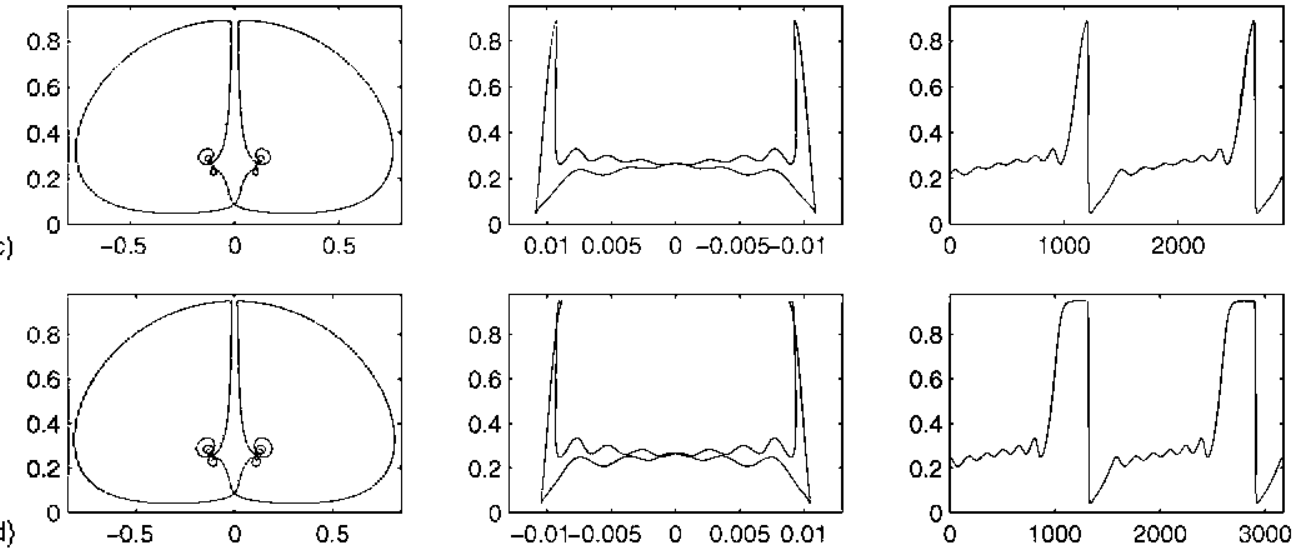

Fig. 28. Case IV. Unstable periodic solutions in the cusp region on branch (d) of Fig. 23, showing the continuous transition from relaxation oscillations involving the states $S^{+}$to oscillations involving the large amplitude states $U^{-}$. (a) $\mu=1.008389$, near ninimum period, (b) $\mu=1.004072$, near the tip of the cusp, (c) $\mu=1.00733$, near the secondary minimum, (d) $\mu=1.00844$, to the right of the secondary minimum.

The divergence of the oscillation period with both increasing and decreasing $\mu$ indicates that all of these branches appear and disappear through global bifurcations (see Fig. 23). To identify these we show in Figs. 29 and 30 high period unstable oscillations on the (d) and (f) branches, one near the initial appearance of each branch and one near its end. In contrast to the (d) branch the (f) branch originates and terminates in global bifurcations involving the origin and the steady states $\pm P_{ \pm}$. However, despite the appearance of Figs. 29 and 30, no heteroclinic connection to a periodic orbit actually occurs. In fact the observed behavior appears to be organized by codimension-two points corresponding to connections between the steady states $P_{ \pm}$and the origin. The figures suggest, and computations confirm, that all the eigenvalues of the fixed points involved are real. However, a more detailed understanding of this remarkable behavior along the lines of refs. [23,21], or indeed in terms of the theory of slow-fast systems, is beyond the scope of this paper.

It is clear that in Case IV the system (26)-(28) exhibits multiple coexisting stable states, some periodic (see Fig. 23), others quasiperiodic or chaotic (see Fig. 21). The latter are readily located between the symmetry-breaking bifurcation on branch (c) and the saddle-node bifurcation on branch (c), and exist on either side of $\mu=1.017$. Since we do not follow unstable tori we cannot identify the transitions that might lead from the various two-frequency states to the observed stable chaotic oscillations. It is likely, however, that each of the periodic oscillations depicted 

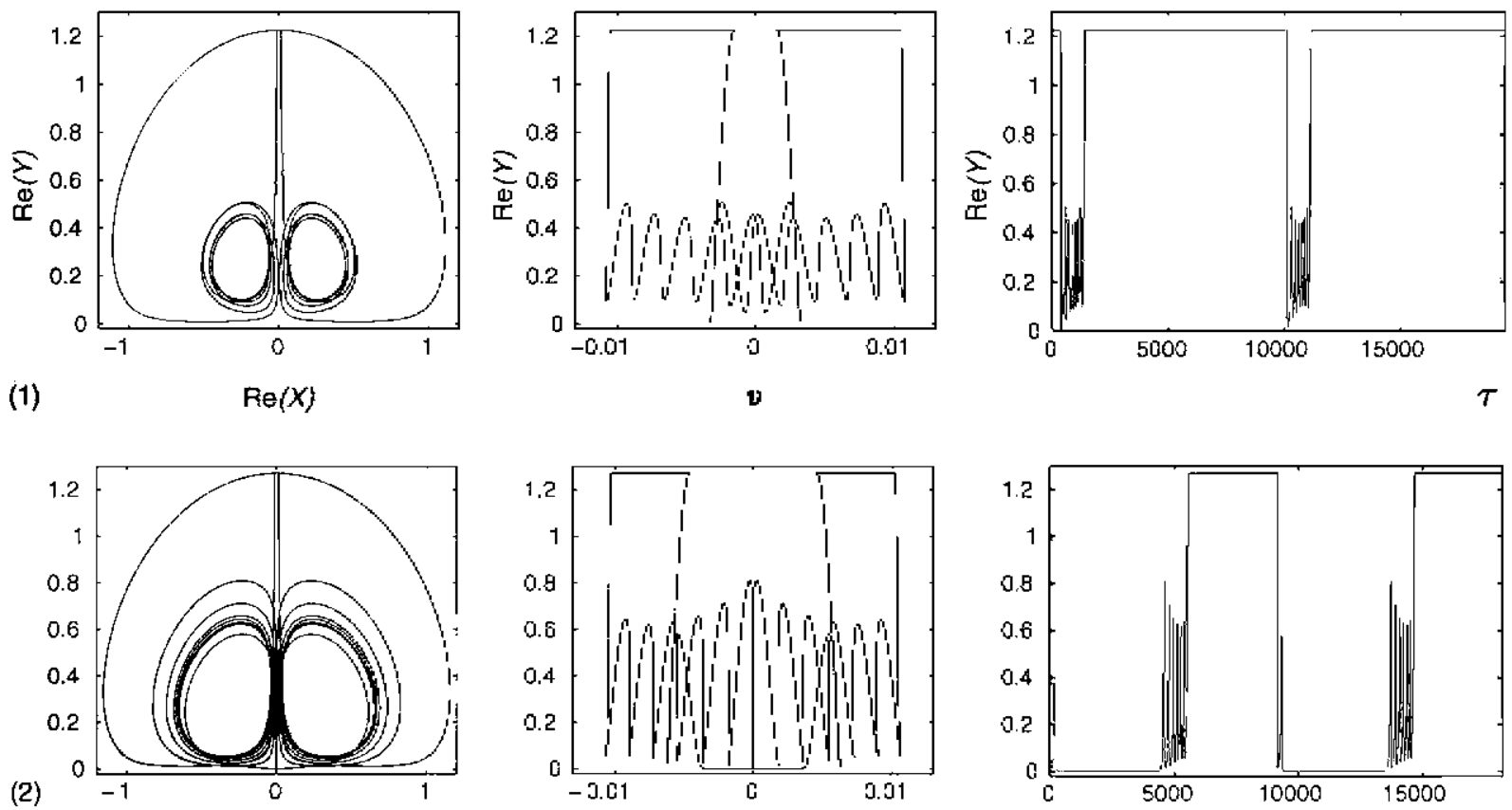

Fig. 29. Case IV. Examples of large period unstable $R_{1}$-symmetric oscillations on branch (d) at the locations indicated in Fig. 23. (1) $\mu=1.0230$, (2) $\mu=1.0258$.

in Fig. 24 bifurcates into chaos, and hence that the example in Fig. $21 \mathrm{~b}$ is but one from a number of coexisting intervals of chaos, cf. [26].

\section{Discussion and conclusions}

In this paper we have examined the dynamics of parametrically driven Faraday waves in slightly elliptical containers on the assumption that (i) the viscosity of the liquid is small (as measured by the dimensionless quantity $\left.C_{\mathrm{g}} \ll 1\right)$ and (ii) the effective Reynolds number Re of the strcaming llow driven in oscillatory boundary layers (defined in Eq. (3)) is also small. Under these conditions we were able to replace the Navier-Stokes-like equation for the mean llow by a single ordinary differential equation, and focused on the interesting case $0<\varepsilon \ll 1$. The mean flow is then only weakly damped, and the resulting equations take the form of a singularly perturbed system. Although the condition for this to be the case, $\lambda_{1} R e^{-1} \ll 1$, provides a compcting constraint on the Rey nolds number, the two requirements are not in contradiction. This is because the small hydrodynamic eigenvalue $\lambda_{1}$ depends on the aspect ratio $R$ of the cylinder and will be small when $R$ is large. The approach we have taken focuses on the role played by the coupling to the streaming flow, but does not attempt to make quantitative predictions for the Faraday system under experimental conditions. Specific applications require the computation of the coupling coefficient $\gamma$ as well as the nonlinear coefficients $\alpha_{1}, \alpha_{2}$ for appropriate meniscus boundary conditions. These remain to be done. For this reason we have chosen to treat Eqs. (26)-(28) as a model system that captures the dominant effects of the coupling to the streaming flow, with $\gamma$ as a free parameter, for fixed detuning $\Gamma$ and ellipticity $\Lambda$. We have 


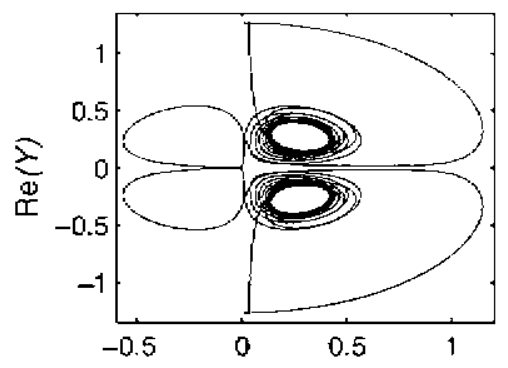

(3)

$\operatorname{Re}(X)$

(4)

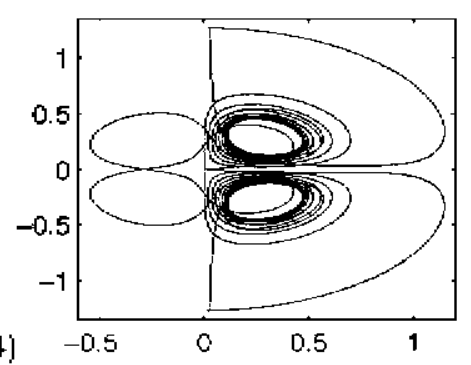

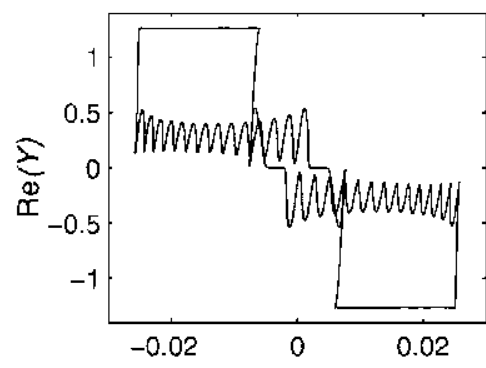

$\boldsymbol{v}$

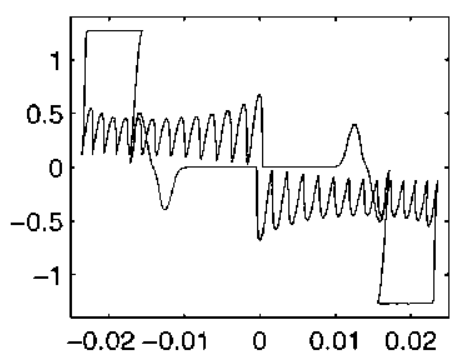

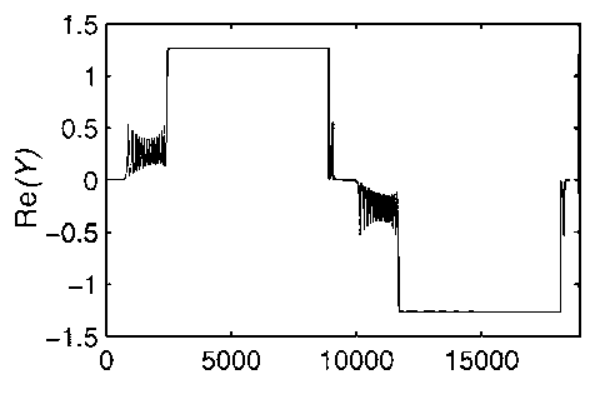

$\tau$

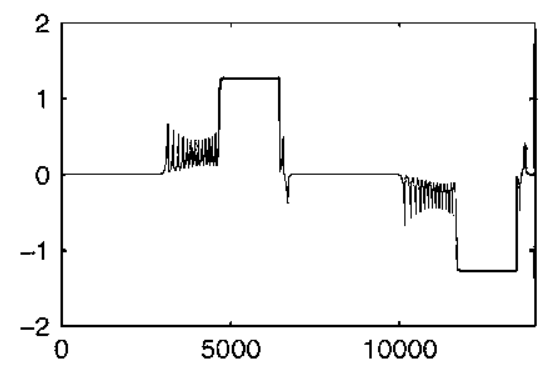

Fig. 30. Case IV. Examples of large period unstable $R_{2}$-symmetric oscillations on branch $(\mathrm{D})$ at the locations indicated in Fig. 23. (3) $\mu=1,02632$, (4) $\mu=1.02680$.

seen that the broken circular symmetry of the system $(\Lambda \neq 0)$ destroys the one-parameter family of standing waves present in a circular container, selecting two slanding waves from this family, with slightly different frequencies and thresholds. As in other problems of this type [36] this results in the competition between two almost degenerate modes, and such interaction often leads to complex dynamics.

We have focused on four distinct cases, three of which were computed for the coefficients $\alpha_{1}, \alpha_{2}$ obtained by Miles for a free contact line [34]. In each of these linear stability theory indicated the absence of any stable steady slates, i.c., the presence of regions in parameter space in which the pure and mixed modes are all unstable. In the first case we found very simple dynamics, organised by a gluing bifurcation of a standard kind, but no chaos. Cases II and III are of greater interest. When $\varepsilon$ is small both these cases exhibit relaxation oscillations with multiple time scales. In Case II these oscillations involved fast transitions between slowly varying steady states called mixed modes, corresponding to periodic standing waves. In the original Faraday system this type of oscillation corresponds to a quasiperiodic mode of oscillation of the system with two quite distinct frequencies. In Case III we located relaxation oscillations of a more complex kind. Here the slow manifold of the amplitude equations is composed of both cquilibria and (symmetric) periodic oscillations, and the resulting time serics (Fig. 19a) appear indistinguishable from the type of (so-called parabolic) bursting behavior exhibited by neurons [44], even though it imvolves visits to saddle-foci. In the Faraday system, the periodic oscillations correspond to mixed mode oscillations in which the contribution of the two orthogonal standing waves oscillates periodically in time (as does their relative phase), and the relaxation oscillations found are oscillations between this complex state and a pure mode standing wave. However, in both cases the basic picture is simple: the system drifts along the slow manifold until it passes a bifurcation, typically a saddle-node bifurcation, where it is forced away from the slow manifold. The resulting fast phase then takes it to another part of the slow manifold and the process repeats. In our system we have seen that oscillations of this type occur when the streaming flow is weakly damped. As a result the streaming flow behaves as a slowly varying parameter, and the system drifts along the slow manifold computed by 'freezing' the mean flow. 
In our system we encountered two twists on this well-known picture. The first centered on the presence of the two rellection symmetries in Eqs. (26)-(28) which permit the presence of periodic oscillations with different types of symmetry; these can in turn undergo symmetry-breaking or symmetry-switching bifurcations. The second centered on the presence of canard explosions characterized by an abrupt change in the amplitude (and in our case symmetry as well) of a periodic orbit. Fig. 6a and b shows an example of each of these orbits in Case II, computed at almost the same value of the parameter $\mu$. The figure shows clearly the dramatic change in the oscillation amplitude as $\mu$ passes through the canard at $\mu=\mu_{\mathrm{e}}$. We were able to demonstrate that the canard in Case II is linked to a subcritical Hopf bifurcation, and established a link between canards and global bifurcations. In particular we argued that there must exist an exponentially small neighborhood of the canard parameter value $\mu_{\mathrm{o}}$ in which the canard is 'unfolded' by any finite value of the small parameter $\varepsilon$, thereby restoring the behavior expected of a global bifurcation. It is this exponential sensitivity to the exact value of $\mu$ that is responsible for the explosive nature of the canard phenomenon [11,2]. In case III we found a whole variety of bursting solutions, characterized by slow drifts along branches of both stable and unstable equilibria and of periodic states, separated by fast transitions between them. Here we located an apparently infinite sequence of lysteretic transitions between asymmetric and $R_{1} R_{2}$-symmetric oscillations occurring in ever narrower intervals in $\mu$ as $\mu$ increases. Much of this behavior could be understood by studying the trajectory near the point of closest approach to the unstable saddles $U^{ \pm}$, and the global behavior of their stable and unstable manifolds, which is in turn inherited from the heteroclinic connection that destroys the oscillations in the fast system. In particular, we saw that the change of symmetry between successive periodic windows is due to an extra half-turn of the trajectory around the unstable steady states as $\mu$ increases. This point of view allowed us to understand why the intervening chaotic intervals become narrower with increasing $\mu$ but the 'symmetry-switching' transitions do not accumulate at a finite value of $\mu$, in contrast to the cascades of symmetry-switching gluing bifurcations that occur in other $D_{2}$-symmetric systems [21].

In Case IV we saw that the primary pure mode branch bifurcates subcritically before turning around towards larger $\mu$. By moving the Hopf bifurcation to direction-reversing waves below this saddle-node bifurcation we made sure that the branch of pure modes $P_{+}$remains unstable above the saddle-node bifurcation. In this case the directionreversing waves created in the Hop bifurcation must also be initially unstable, and only acquire stability at larger $\mu$ at a torus bifurcation. When this bifurcation is supercritical the resulting tonus branch bifurcates towards smaller values of $\mu$ and is at least initially stable. The branch terminates in a global bifurcation involving the pure modes and this bifurcation is typically associated with chaos $[17,24,25]$. Thus, in this instance the primary bifurcation gives rise to either chaotic or quasiperiodic oscillations even though it is a steady state bifurcation. The same mechanism has been seen in other systems, and is responsible for the presence of chaos at onset of natural doubly diffusive convection [7] and for the presence of a three-frequency state called a repeated transient at onset of binary fluid convection [6]. However, a more detailed look at this case revealed a plethora of additional branches of periodic orbits, which start and end in global bifurcations, and acquire stability through torus bifurcations. These periodic orbits have a highly unusual appearance which we have traced to the separation between the time scales for the cvolution of the waves and of the mean flow, specifically the requirement $0<\varepsilon \ll(I-A)^{2} \sim 10^{-3}$. In particular, we found that the unusual appearance of the oscillations is the consequence of delayed loss of stability that occurs as a result of a slow passage through a Hopf bifurcation in a system with symmetry. Theory (cf. [2]) shows that the delay lasts for a time of order $1 / \varepsilon$; during this time the solution drifts along the unstable manifold of equilibria, much as in a canard, although the resulting behavior is not canard-like and in particular has quite different dependence on the parameters $\mu$ and $\varepsilon$. Note that since the Hopf bifurcations are subcritical the unstable periodic orbits created at the bifurcation are not involved in this behavior, cf. [20].

The present system differs in an important aspect from the most familiar systems exhibiting canards in having a pair of reflection symmetries. These symmetries have an important effect on the transitions, and in particular one can have canards on branches of symmetric oscillations as well as nonsymmetric ones. Related behavior is present when two identical oscillators are symmetrically coupled. The resulting system can undergo relaxation oscillations with both oscillators oscillating in phase (a symmetric oscillation) or exactly out of phase (an asymmetric oscillation). 
Examples include coupled neurons [18] and coupled continuously stirred tank reactors [4,5], but the transition between these two oscillation types has not hitherto been systematically investigated. This is an interesting topic for future investigation.

The results of this paper demonstrate that the inclusion of the streaming flow in the amplitude equations describing Faraday waves in a nearly circular domain has dramatic consequences for the range of available dynamical behavior. In particular, its presence is responsible for relaxation oscillations, an unusual phenomenon in fluid dynamics, and one that could be confirmed in experiments. Of the four cases considered, Case IV is closest to the conditions required for the validity of Eqs. (26)-(28) since both $\Gamma$ and $\Lambda$ are small, and the magnitude of the streaming flow remains small as well. Yet despite this its effects remain dramatic (cf. Fig. 20). In forthcoming work, we shall examine these transitions in greater detail, and shall explore the analogues of this behavior in the partial differential Eqs. (1)-(6) as well.

\section{Acknowledgements}

This work was supported in part by EPSRC grant GR/R52879/01 and by an EPSRC visitor grant at Leeds University $(\mathrm{MH})$. Partial support from the NASA Microgravity Sciences Program under grants NAG3-2152 and NNC()4GA47G is also gratefully acknowledged. We thank J. Guckenheimer, M. Krupa, J. Mochlis, J. Porter and M. Wechselberger for several helpful discussions.

\section{References}

[1] H. Arbell, I. Fincherg, Pattern formation in two-frequeney forced parametric waves, Phys. Rev. F, 65 (2002) 036224.

[2] V.I. Amol'd, V.S. Afrajmovich, Yu.S. Il'yashenko, I.P. Shil'nikov, Bifurcation theory and eatastrophe theory, in: V.I. Amol'd (F.d.), Encyclopaedia of Mathematical Sciences, wol. 5, Springer-Verlag, New York, 1994.

[3] S.M. Baer, T. Emeux, Singular IIop bifurcation to relixation oscillations, SIAM J. Appl. Math. 46 (1986) 721-739.

[4] K. Bar-Eli, Coupling of identical oscillators, J. Plyys. Chem. 94 (1990) 23682374.

[5] K. Bar-Eli, M. Brons, Period lengthening near the end of oscillations in chemical systems, J. Plys. Chem. 94 (1990) 71707177.

[6] O. Batiste, E. Knobloch, I. Mercader, M. Net, Simulations of oscillatory binary fluid convection in large aspect ratio containers, Phys. Rev. f. $65(2001) 016303$.

17] A. Bergeon, E. Knobloch, Natural doubly diffusive convection in three-dimensional enclosures, Phys. Fluids 14 (2002) 3233-3250.

[8] M. Brons, K. Bar-Eli, Canard explosions and excitation in at model of the Belousov-Zhabotinsky reaction, J. Phys. Chem. 95 (1991) 87068713.

[9] G. Dangelmayr, E. Knobloch, Hopf bifurcation with broken circular symmetry, Nonlinearity 4 (1991) 399427.

[10] S. Douady, S. Fauve, O. 'Ihual, Oscillatory phase modulation of parametrieally foreed surface waves, furophys. 1,tt. 10 (1989) 309315 .

[11] W. Ecklaus, Relaxation oscillations, including a standard chase on French ducks, Lecture Noles in Mathematies, vol. 985, Springer-Verlag, New York, 1983, pp. 449-494.

[12] W.S. Edwards, S. Fauve, Patterns and quasi-patterns in the Faraday experiment, J. Fluid Mech. 278 (1994) 123148.

[13] N. Fenichel, Geometric singular perturbation theory for ordinary differential equations, J. Diff. Eq. 31 (1979) 5398

[14] M. Funakoshi, S. Inoue, Surface waves due to resonant horizontal oseillation, J. Fluid Mech. 192 (1988) $219-247$.

[15| P. Glendinning, Global structure of homoclinie bifurcations: a combinatorial approach, Phys. Lett. A 141 (1989) 391-396.

[16] P. Glendiming, J. Abslagen, T. Mullin, Imperfect homoclinic bifurcations, Phys. Rev. E 64 (2001) 036208.

[17] J. Guckenheimer, On a codimension-two bifurcation, in: D.A. Rand, L.S. Young (Eds.), Dynamical systems and turbulence, Lecture Notes in Mathematics, vol. 898, Springer-Verlag, New York, 1981, pp. 99142.

[18] J. Guckenheimer, K. Hoffman. W. Weckesser, Numerical computation of canards, Int. J. Bif. Chaos 10 (2000) $\left.266^{\circ}\right)-2687$.

[19] J. Guckenheimer, P. Holmes, Nonlinear Oseillations, I)ynamical Systems, and Bifureations of Vector Fields, second ed., Springer-Verlag, New York, 1986.

[20] J. Guckenheimer, A.R. Willms, Asymptotic analysis of subcritical IIopf-homoclinic bifurcation, Physica D 139 (2000) $195-216$.

[21] M. Higuera, J. Porter, E. Knobloch, Heteroclinic dynamics in the nonlocal parametrically driven nonlinear Schrödinger equation, Physsica D $162(2002) 155187$. 
[22] M. Higuera, J.M. Vega, E. Knobloch, Coupled amplitude-streaming flow equations for nearly inviscid Faraday waves in small aspect ratio containers, J. Nonlinear Sci. 12 (2002) 505551.

[23] P. Hirschberg, F.. Knobloch, The Shil'nikov-Hopf bifurcation, Physica D 62 (1993) 202-216.

[24] V. Kirk, Breaking of symmetry in the saddle-node Hopf hifurcation, Phys. 1.ett. A 154 (1991) 243-248.

[25] V. Kirk. Merging of resonince tongues, Physica D 66 (1993) 267-281.

[26] B. Krauskopl, S. Wieczorek, Accumulating regions of winding periodic orbits in optically driven lasers, Physica D 173 (2002) $97-113$.

[27] A. Kudrolli, J.P. Gollub, Pattems and spatio-temporal chaos in parametrically forced surface waves: a systematic survey at large aspect ratio, Physica D 97 (1997) 133154.

[28] A. Kudrolli, B. Pier, J.P. ('ollub, Superlattice patterns in surfaee waves, Physica D 123 (1998) $9-111$.

[29] A.S. I.andsherg, F. Knobloch, Direction-reversing traveling waves, Phys. I ctt. A 159 (1991) $17-20$.

|30| V. Lapuerta, C. Martel, J.M. Vega, Weakly dissipative Faraday waves in 2D large aspect ratio annuli, Physica D 173 (2002) $178-203$.

[31] M.S. Longuet-Higgins, Mass transport in water waves, Phil. Trans. Roy. Soc. $A 245$ (1953) 535581.

[32] C. Martel, J.A. Nicolás, J.M. Vega, Surface-wave damping in a brimful circular cylinder, J. Fluid Mech. 360 (1998) 213228 (see also Corrigendum 373 (I998) 379).

[33] F.. Martin, C. Martsl, J.M. Vega, Drift instability of standing Faraday waves, J. Fluid Mech. 467 (2002) 57-79.

[34] J.W. Miles, Internally resonant surface waves in a circular cylinder, J. Fluid Mech. 149 (1984) 1-14.

[35] J. Moehlis, Canards in a surface oxidation reaction, J. Nonlinear Sci. 12 (2002) 319-345.

[36] J. Moehlis, E. Knobloch, Forced symmetry breaking as a mechanism for bursting, Phys. Rev. Lett. 80 (1998) 53295332.

[37] J. Porter, M. Silber, Broken symmetries and pattem formation in two-frequency forced Faraday waves, Phys. Rev. Lett. 89 (2002) 084501.

[38] A.M. Rucklidge, P.C. Matthews, Analysis of the shearing instability in nonlinear eonvection and magnetoconvection, Nonlinearity 9 (1996) 311-351.

[39] A.M. Rucklidge, M. Silber, J. Fineberg, Spatial period-multiplying inslabilities of hexagonal Faraday waves, in: J. Buescu, S.B.S.D. Castro, A.P.S. Dias, I.S. Labouriau (Eds.), Bifurcations, Symmetry and Patlems, Birkhäuser, Basel, 2003, pp. 101-114.

[40] H. Schlichting, Berechnung ebener periodischer Grenzschichtströmungen, Phys. Z. 33 (1932) 327335.

[41] D. Terman, Chaotic spikes arising from a model of bursting in excitable membranes, SIAM J. Appl. Matl. 51 (1991) 14181450.

[42] D.P. 'Tse, A.M. Rueklidge, R.B. Hoyle, W. Silher, Spatial period-multiplying instabilities of hexagonal Faraday waves, Physica I) 146 (2000) $367-387$.

[43] J.M. Vega, E. Knobloch, C. Martel, Nearly inviscid Faraday waves in annular containers of moderately large aspect ratio, Physica D 154 (2001) 313-336

[44] X.J. Wang, J. Rinzel, Oscillatory and bursting properties of neurons, in: M.A. Arbib (Ed.), Brain Theory and Neural Networks, MIT Press, Cambridge, MA, 1995.

[45] S. Wiggins, Global Bifurcations and Chaos, Springer-Verlag, New York, 1988.

[46] R.W. Wittenberg, P. Holmes, The limited effectiveness of normal forms: A eritical review and extension of local bifurcation studies of the Brusselator PDE, Physica D 100 (1997) 1-40.

[47] M.K.S. Yeung, S.II. Strogatz, Nonlinear dynamics of a solid-state laser with injection, Phys. Rev. E 58 (1998) $4421-4435$ (see also Corrigendum $61(2000) 2154)$. 